\title{
Search for top squarks in final states with two top quarks and several light-flavor jets in proton-proton collisions at $\sqrt{s}=13 \mathrm{TeV}$
}

\author{
A. M. Sirunyan et al. \\ (CMS Collaboration)
}

(Received 13 February 2021; accepted 28 June 2021; published 20 August 2021)

\begin{abstract}
Many new physics models, including versions of supersymmetry characterized by $R$-parity violation (RPV), compressed mass spectra, long decay chains, or additional hidden sectors, predict the production of events with top quarks, low missing transverse momentum, and many additional quarks or gluons. The results of a search for new physics in events with two top quarks and additional jets are reported. The search is performed using events with at least seven jets and exactly one electron or muon. No requirement on missing transverse momentum is imposed. The study is based on a sample of proton-proton collisions at $\sqrt{s}=13 \mathrm{TeV}$ corresponding to $137 \mathrm{fb}^{-1}$ of integrated luminosity collected with the CMS detector at the LHC in 2016-2018. The data are used to determine best fit values and upper limits on the cross section for pair production of top squarks in scenarios of RPV and stealth supersymmetry. Top squark masses up to 670 (870) GeV are excluded at 95\% confidence level for the RPV (stealth) scenario, and the maximum observed local signal significance is 2.8 standard deviations for the RPV scenario with top squark mass of $400 \mathrm{GeV}$.
\end{abstract}

DOI: 10.1103/PhysRevD.104.032006

\section{INTRODUCTION}

Supersymmetry [1,2] (SUSY) is an extension of the standard model (SM) that may provide a solution to the gauge hierarchy problem [3]. In the SUSY framework, quadratically divergent radiative corrections to the Higgs boson mass parameter, dominated by loops involving the top quark, are canceled by loops with bosonic top quark superpartners (top squark, $\tilde{t}$ ). To avoid fine-tuning, the lightest $\tilde{t}$ and the superpartners of the Higgs bosons (Higgsinos) must have masses near the weak scale [3-8] and could therefore have nonnegligible production cross sections at the CERN Large Hadron Collider (LHC).

Most searches for the $\tilde{t}$ look for an excess of events with large missing transverse momentum $p_{\mathrm{T}}^{\text {miss }}$ originating from the undetected lightest SUSY particle (LSP) produced in $\tilde{t}$ decays. It is typical in these searches to assume that the LSP is the lightest neutralino $\tilde{\chi}_{1}^{0}$, which is stable if $R$-parity [9] is conserved. However, it has been shown [10-12] that this search strategy is not sensitive to well-motivated SUSY models that predict signatures with low $p_{\mathrm{T}}^{\text {miss }}$ in models with gauge mediated SUSY breaking [13], compressed mass spectra [14,15], hidden valleys [16], or other

${ }^{*}$ Full author list given at the end of the article.

Published by the American Physical Society under the terms of the Creative Commons Attribution 4.0 International license. Further distribution of this work must maintain attribution to the author(s) and the published article's title, journal citation, and DOI. Funded by SCOAP ${ }^{3}$. mechanisms. As searches performed at the LHC using events with high $p_{\mathrm{T}}^{\text {miss }}$ set ever more stringent lower bounds on the $\tilde{t}$ mass [17-22], searches for low- $p_{\mathrm{T}}^{\text {miss }}$ alternatives become increasingly important.

Models of $R$-parity violating (RPV) SUSY produce low$p_{\mathrm{T}}^{\text {miss }}$ signatures by providing a mechanism for the LSP, in this case $\tilde{\chi}_{1}^{0}$, to decay. Among other couplings, RPV SUSY includes a trilinear Yukawa coupling between quarks and squarks that allows the $\tilde{\chi}_{1}^{0}$ to decay into three quarks via an off shell squark [9]. These couplings are typically referred to as $\lambda_{i j k}^{\prime \prime}$ where $i, j$, and $k$ specify the generations of the participating (s)quarks. The benchmark RPV model used in this analysis is illustrated in Fig. 1. The $\tilde{t}$ decays in the typical way into a top quark and a $\tilde{\chi}_{1}^{0}$, and the $\tilde{\chi}_{1}^{0}$ undergoes an RPV decay via nonzero $\lambda_{112}^{\prime \prime}$ into three light-flavor quarks, $\tilde{\chi}_{1}^{0} \rightarrow u d s$. However, since this analysis does not distinguish between jets originating from quarks of the first and second generation, our results are more broadly applicable to any RPV model with coupling $\lambda_{a b c}^{\prime \prime}$ with $a, b, c \in\{1,2\}$.

Stealth SUSY models $[12,23,24]$ introduce a new hidden "stealth" sector of light particles with small or absent couplings to the SUSY breaking sector and finite couplings to the visible sector. Because of the weak connection to the SUSY breaking sector, SUSY is approximately conserved in the stealth sector, resulting in stealth particles that are nearly mass-degenerate with their superpartners. Production and decay of stealth particles via interactions with visible particles can be achieved through a variety of 

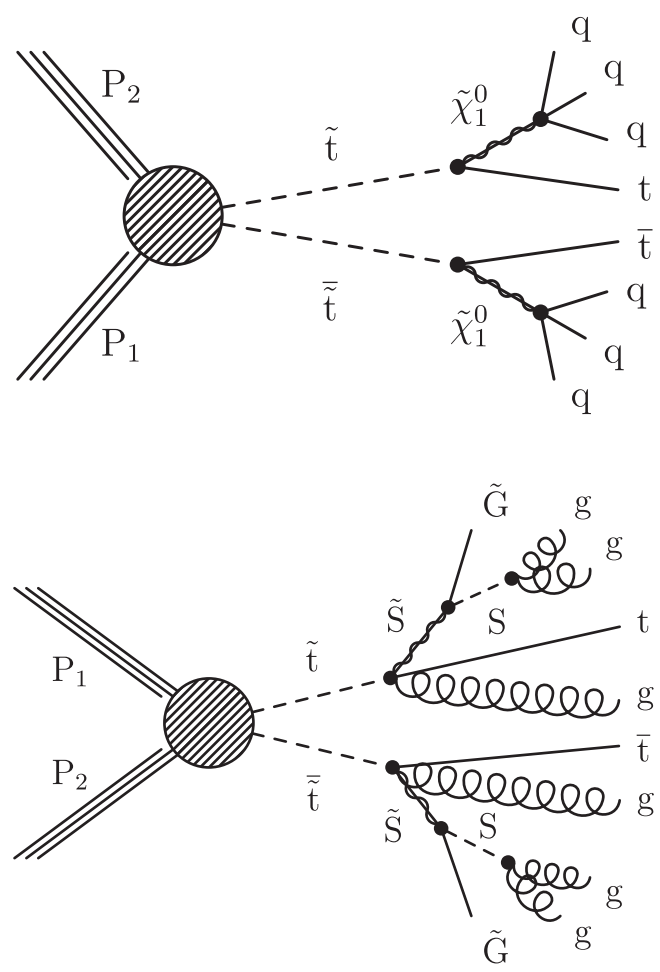

FIG. 1. Diagrams of top squark pair production with decays to top quarks and additional light-flavor quarks for the RPV SUSY model (upper) and with decays to top quarks and gluons for the stealth $S Y \bar{Y}$ model (lower).

"portals" including mediation by the Higgs boson or new particles at a higher mass scale. The benchmark stealth SUSY model used in the interpretation of the results of this search (stealth $S Y \bar{Y}$ ) [24] assumes a minimal stealth sector containing only one scalar particle $S$ with even $R$-parity and its superpartner $\tilde{S}$, both of which are singlets under all SM interactions, and a portal mediated by loop interactions involving a new vectorlike messenger field $(\mathrm{Y})$, the gluon $(g), \tilde{\chi}_{1}^{0}, S$, and $\tilde{\mathrm{S}}$. Decays of the $\tilde{t}$ in the stealth $S Y \bar{Y}$ model are illustrated in Fig. 1. Each $\tilde{t}$ decays to a gluon, top quark, and $\tilde{\mathrm{S}}$, with subsequent decays of $\tilde{\mathrm{S}}$ to $S$ and a gravitino $\tilde{G}$ and $S$ to jets via $S \rightarrow g g$. Because of the small mass splitting between the $S$ and $\tilde{\mathrm{S}}$, as well as the small $\tilde{G}$ mass, the undetected $\tilde{G}$ carries away very little momentum. Thus, the stealth $S Y \bar{Y}$ model shares the general feature of all stealth SUSY models in that it naturally produces a low$p_{\mathrm{T}}^{\text {miss }}$ signature without $R$-parity violation or a special tuning of sparticle masses.

The RPV and stealth $S Y \bar{Y}$ models are characterized by the masses of the particles and branching fractions in the decay chain. In the benchmark RPV model, we take the $\tilde{\chi}_{1}^{0}$ mass to be $100 \mathrm{GeV}$. For the benchmark stealth $S Y \bar{Y}$ model, the critical small $\tilde{\mathrm{S}}-S$ mass splitting is held constant at $10 \mathrm{GeV}$, and we assume a $\tilde{S}$ mass of $100 \mathrm{GeV}$ and a $\tilde{G}$ mass of $1 \mathrm{GeV}$. For both models, a range of $\tilde{t}$ masses $\left(m_{\tilde{t}}\right)$ are considered from 300 to $1400 \mathrm{GeV}$, and all decays described above are assumed to be prompt with unity branching fractions.

In this paper, we describe a search for $\tilde{t}$ pair production followed by the decay of each $\tilde{t}$ into a top quark and three light-flavor jets via the benchmark RPV and stealth $S Y \bar{Y}$ models described above. This is the first search of its kind at the LHC. Previous searches for RPV $\tilde{t}$ decays focused on final states with dijet resonances [25,26], lepton-jet resonances [27,28], intermediate leptonic chargino decays [29], or final states with many $b$ quarks [30]. Previous searches for stealth SUSY targeted superpartners of light-flavor quarks with decays into gauge bosons and jets [31,32]. Measurements of the $t \bar{t}$ differential production cross section have been reinterpreted in the context of RPV and stealth SUSY $[24,33]$ and were found to yield weak constraints for the models considered in this paper.

Before describing each step in more detail in subsequent sections, we provide an overview of the analysis strategy here. The main distinguishing feature of the signals in this analysis, in addition to the presence of two top quarks, is high jet multiplicity $\left(N_{\text {jets }}\right)$. The SM backgrounds arise through processes including top quark pair production $(t \bar{t})$, multijet production from quantum chromodynamics (QCD), production of $t \bar{t}$ in association with SM weak gauge bosons or additional top quarks $(t \bar{t}+X)$, production of weak gauge bosons, and single top quark production (other). These SM processes all include additional jets from initial- and final-state radiation (ISR and FSR). The QCD background is primarily suppressed by requiring the presence of exactly one charged lepton ( $e$ or $\mu$ ) arising from the leptonic decay of a top quark. Backgrounds that do not produce any top quarks are suppressed by requiring the presence of at least one jet identified as arising from the fragmentation of a bottom quark ( $b$-tagged jet), and additionally that the invariant mass of the lepton and a $b$-tagged jet be consistent with the presence of a top quark.

The signal is distinguished from the dominant and irreducible $t \bar{t}$ background by means of a neural network (NN) trained to recognize differences in the spatial distribution of jets and decay kinematic distributions between signal and $t \bar{t}$ background events. Events are divided into 24 categories based on their $\mathrm{NN}$ score $\left(S_{\mathrm{NN}}\right)$ and $N_{\text {jets }}$; categories with higher (lower) $S_{\mathrm{NN}}$ and $N_{\text {jets }}$ tend to be signal enriched (depleted). We perform a simultaneous fit to the number of events in data in $S_{\mathrm{NN}}$ and $N_{\text {jets }}$ categories to estimate the total numbers of $t \bar{t}$ and potential signal events present in the data, as well as the distribution of $t \bar{t}$ events in $S_{\mathrm{NN}}$ and $N_{\text {jets }}$ categories. The $\mathrm{NN}$ output is designed to have no dependence on $N_{\text {jets }}$, so that the $N_{\text {jets }}$ distribution of $t \bar{t}$ events can be constrained in the fit to be the same for all $S_{\mathrm{NN}}$ categories. This requirement for $t \bar{t} N_{\text {jets }}$ shape invariance is important for the analysis and will be discussed throughout the paper. 
This paper is organized as follows. We introduce the CMS detector and methods for event reconstruction and selection in Sec. II. Samples of simulated events are described in Sec. III. The estimation and modeling of SM backgrounds are explained in Sec. IV, and the description of the treatment of systematic uncertainties is in Sec. V. Finally, the results and their interpretation are in Sec. VI, followed by the summary in Sec. VII.

\section{EXPERIMENTAL TECHNIQUES}

The search is performed using a data sample of protonproton $(p p)$ collisions at $\sqrt{s}=13 \mathrm{TeV}$, corresponding to an integrated luminosity of $137 \mathrm{fb}^{-1}$, collected in 2016-2018 with the CMS detector at the LHC. Data and simulation samples from four periods $(2016,2017,2018 \mathrm{~A}, 2018 \mathrm{~B})$ are treated separately in order to address variations in detector and LHC conditions. Data from 2018 are divided into two samples (2018A and 2018B), with 2018B corresponding to the period when a detector malfunction prevented readout from $3 \%$ of the hadron calorimeter. In this section, we define reconstructed physics objects and describe the selection criteria for events in the signal region (SR) and the control region $(\mathrm{CR})$ of the analysis.

The central feature of the CMS apparatus is a superconducting solenoid of $6 \mathrm{~m}$ internal diameter, providing a magnetic field of $3.8 \mathrm{~T}$. Within the solenoid volume are a silicon pixel and strip tracker, a lead tungstate crystal electromagnetic calorimeter, and a brass and scintillator hadron calorimeter, each composed of a barrel and two end cap sections. Forward calorimeters extend the pseudorapidity coverage provided by the barrel and end cap detectors. Muons are detected in gas-ionization chambers embedded in the steel flux-return yoke outside the solenoid. A more detailed description of the CMS detector, together with a definition of the coordinate system used and the relevant kinematic variables, can be found in Ref. [34].

The CMS trigger system is described in Ref. [35]. Events are selected using triggers that require the presence of at least one electron or one muon. The minimum transverse momentum $p_{\mathrm{T}}$ threshold is 27 (35) GeV for electrons and 24 (24) GeV for muons in 2016 (2017-2018). The triggers at these thresholds require the lepton to be isolated from tracks and calorimeter deposits in the detector. Events may also be selected from single-lepton triggers with higher $p_{\mathrm{T}}$ thresholds, $115 \mathrm{GeV}$ for electrons and $50 \mathrm{GeV}$ for muons, with no isolation requirements. The combined trigger efficiency varies from $80 \%$ for leptons with $p_{\mathrm{T}}$ close to the lower thresholds to greater than $95 \%$ for leptons with $p_{\mathrm{T}}>120 \mathrm{GeV}$.

Events are reconstructed using the particle-flow (PF) algorithm [36], which reconstructs particles in an event using an optimized combination of information from the various elements of the CMS detector and identifies each as a photon, electron, muon, charged hadron, or neutral hadron. These particles are further clustered into jets as described below.

The reconstructed vertex with the largest value of summed physics-object $p_{\mathrm{T}}^{2}$ is taken to be the primary $p p$ interaction vertex, where the physics objects are the jets, clustered using the anti- $k_{\mathrm{T}}$ algorithm [37,38] with the charged-particle tracks assigned to the vertex as inputs, and the associated missing transverse momentum, taken as the negative vector sum of the $p_{\mathrm{T}}$ of those jets [39]. Charged-particle tracks associated with vertices from other $p p$ interactions (pileup) are removed from further consideration. The primary vertex is required to lie within $24 \mathrm{~cm}$ of the interaction point along the beam axis, and within $2 \mathrm{~cm}$ in the plane transverse to the beam axis.

Electrons and muons must satisfy $p_{\mathrm{T}}>30 \mathrm{GeV}$ and $|\eta|<2$.4. For the analysis of the 2017 and 2018 data, the electron $p_{\mathrm{T}}$ threshold is increased to $37 \mathrm{GeV}$ to account for the higher trigger threshold. The lepton identification requirements are the "tight" criteria for electrons [40] and the "medium" criteria for muons [41]. Leptons must be isolated within a cone of radius $R=\sqrt{(\Delta \phi)^{2}+(\Delta \eta)^{2}}$ that scales as $1 / p_{\mathrm{T}}$ between a maximum of 0.2 for leptons with $p_{\mathrm{T}}<50 \mathrm{GeV}$ and a minimum of 0.05 for lepton $p_{\mathrm{T}}>200 \mathrm{GeV}$ [42].

Jets are clustered from the reconstructed PF particles using the anti- $k_{\mathrm{T}}$ algorithm with a distance parameter of 0.4 . Criteria are applied to remove events with jets arising from instrumental effects or reconstruction failures [43,44]. The reconstructed jet energies are corrected for the nonlinear response of the detector $[45,46]$ and for contributions from neutral hadrons from pileup [47]. Jets are required to have $p_{\mathrm{T}}>30 \mathrm{GeV}$ and $|\eta|<2.4$. Jets overlapping with a selected lepton within a cone of radius $R=0.4$ are removed. A neural network-based algorithm [48] is used to identify $b$ quark jets; for jets with $p_{\mathrm{T}}$ around $30 \mathrm{GeV}$, the algorithm has an efficiency of $65 \%$ and a misidentification rate for light-flavor jets (including gluon jets) of $1 \%$.

In addition to the trigger and vertex criteria above, events in the SR must contain exactly one isolated electron or muon and at least seven jets, at least one of which should be $b$ tagged. Samples with seven and eight jets include a small number of expected signal events but are included in the SR to constrain the background. To further reject the QCD background, we require the scalar sum of jet $p_{\mathrm{T}}\left(H_{\mathrm{T}}\right)$ to

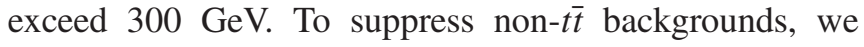
require the invariant mass of the system formed by the $b$-tagged jet and the lepton to be between 50 and $250 \mathrm{GeV}$. If there is more than one $b$-tagged jet in the event, the invariant mass of each $b$-tagged jet and the lepton is considered, and at least one combination is required to meet the above criterion. No requirement is made on the event $p_{\mathrm{T}}^{\text {miss }}$.

In addition to the SR, a signal-depleted control region (CR) dominated by QCD background is defined with the dual purpose of determining the QCD contribution to the 
SR and verifying the important assumption of $t \bar{t} N_{\text {jets }}$ shape invariance with $S_{\mathrm{NN}}$. Despite being dominated by QCD background, the CR is useful for confirming $t \bar{t} N_{\text {jets }}$ shape invariance because many of the jets used as inputs to the $\mathrm{NN}$ arise from QCD radiation, which is common to the $t \bar{t}$ and QCD backgrounds; this claim is verified in Sec. V. The $\mathrm{CR}$ is defined similarly to the SR with the differences being that the lepton is required to be a muon; the muon is required to fail the SR isolation requirement; there is no requirement for a $b$-tagged jet, nor on the invariant mass of the lepton and $b$-tagged jet; the only trigger used is the high-threshold muon trigger without an isolation requirement; and the muon $p_{\mathrm{T}}$ threshold is $55 \mathrm{GeV}$.

\section{SIMULATED EVENT SAMPLES}

Simulated event samples are used in the estimation of the expected number of SM background and signal events passing the SR selection. Top quark pair and single top quark events produced in the $t$ channel are generated with the next-to-leading-order (NLO) POWHEG v2.0 [49-53] generator, while single top quark events in the tW channel are generated with POWHEG v1.0 [52]. Single top quark production in the $s$ channel, as well as rare SM processes such as $t \bar{t} Z$ and $t \bar{t} W$ are generated at NLO accuracy with the MadGraph 5_aMC@NLO v2.2.2 program. The MadGraph 5_aMC@NLO v2.2.2 generator $[54,55]$ is used in the leadingorder (LO) mode to simulate QCD and $W+$ jets events.

For the signal, top squark pair production events are generated using MadGraph 5_aMC@NLO in LO mode, including up to two additional partons in the matrix element calculation. The top squarks are decayed using PYTHIA v8.212 (2016) or 8.226 (2017-2018) [56] according to the signal models described in Sec. I. The signal production cross section $\left(\sigma_{\tilde{t} \tilde{t}}\right)$ is calculated as a function of $m_{\tilde{t}}$ using approximate next-to-NLO (NNLO) plus nextto-next-to-leading-logarithm (NNLL) calculations [57,58].

The generation of these processes is based on either LO or NLO parton distribution functions (PDFs) using NNPDF3.0 [59] for the simulated samples corresponding to 2016 detector conditions and using the NNLO PDF sets from NNPDF3.1 [60] for the 2017 and 2018 simulated samples. Parton showering and hadronization are simulated with PYTHIA using underlying event tune CUETP8M1 [61] for 2016 samples, except for $t \bar{t}$ production which used tune CUETP8M2T4 [62], or PYTHIA with tune CP5 (CP2) [63] for all 2017 and 2018 background (signal) samples. To model the effects of pileup, simulated events are generated with a nominal distribution of $\mathrm{pp}$ interactions per bunch crossing and then reweighted to match the corresponding distribution in data. The CMS detector response is simulated using a GEANT4-based model [64], and event reconstruction is performed in the same manner as for collision data. The most precise cross section calculations available are used to normalize the SM simulated samples, corresponding to NLO or NNLO accuracy in most cases [54,65-71].

The simulation is corrected to eliminate small discrepancies between data and simulation in the trigger efficiency, lepton selection efficiency, and $b$ tagging efficiency. Analysis-specific corrections for the $H_{\mathrm{T}}$ distribution in $t \bar{t}$ simulation, parametrized as functions of $N_{\text {jets }}$ and $H_{\mathrm{T}}$, are obtained in a signal-depleted sample identical to the $\mathrm{SR}$, except for the requirement $5 \leq N_{\text {jets }} \leq 7$. Events with $N_{\text {jets }}=7$ are common to the SR, but as mentioned above, this sample has low signal contamination. The correction is parametrized with an exponential function in $H_{\mathrm{T}}$ with parameters depending linearly on $N_{\text {jets }}$ in order to extend the correction into the $N_{\text {jets }}>7 \mathrm{SR}$. The $H_{\mathrm{T}}$ correction is small at low $H_{\mathrm{T}}$ and $20 \%-40 \%$ at $H_{\mathrm{T}}=1500 \mathrm{GeV}$, depending on $N_{\text {jets }}$.

\section{BACKGROUND ESTIMATION}

Simulated background events passing the SR selection requirements predominantly originate from $t \bar{t}$ production, with contributions of less than $10 \%$ from QCD, and a few percent from the remaining minor backgrounds including $t \bar{t}$ production in association with a vector boson, single top quark production, and $W+$ jets.

As introduced in Sec. I, the crux of the analysis is to estimate the dominant $t \bar{t}$ background in four bins of $S_{\mathrm{NN}}$ and six $N_{\text {jets }}$ bins using a simultaneous binned maximumlikelihood fit constraining the $t \bar{t} N_{\text {jets }}$ shape to be the same in all $S_{\mathrm{NN}}$ categories. Event yields, as well as the $N_{\text {jets }}$ and $S_{\mathrm{NN}}$ distributions, are fixed at values determined from a signal-depleted data control sample for the QCD background and from simulation for the minor backgrounds, as described later in this section. The yield and $N_{\text {jets }}$ shape of the $t \bar{t}$ background, along with the signal strength, are determined in the fit; signal strength is defined as the ratio of the fit signal event yield to the one predicted by SUSY.

The $\mathrm{NN}$ is trained to discriminate between signal and $t \bar{t}$ background by exploiting differences in the event shape and distributions of the kinematic variables. The gradient reversal technique [72] is used to minimize dependence of the $\mathrm{NN}$ output on $N_{\text {jets }}$, as required by the primary assumption that the $t \bar{t} N_{\text {jets }}$ shape is the same in all $S_{\mathrm{NN}}$ categories. All NN input variables are computed in an approximate center-of-mass frame defined by all jets in the event with $p_{\mathrm{T}}>30 \mathrm{GeV}$ and $|\eta|<5$. The NN input variables include the four-vector components for the seven jets in the event with the highest momentum in the centerof-mass frame, the four-vector components of the lepton in the event, the second through fifth Fox-Wolfram moments [73] normalized by the first moment, and the three eigenvalues of the sphericity tensor [74] normalized by the sum of the eigenvalues. The Fox-Wolfram moments and sphericity tensor eigenvalues, which are computed from the same seven highest momentum jets, quantify the 
distribution of jet energy in the event, which tends to be more spherical for signal $\tilde{t}$ pair production than for the $t \bar{t}$ background.

For the NN training, simulated $t \bar{t}$ events are used for the background sample, and a mixture of RPV and stealth $S Y \bar{Y}$ simulated events with $m_{\tilde{t}}$ from $350-850 \mathrm{GeV}$ is used as the signal sample. In this way, the NN can identify common features among all signal samples ensuring a search with broad sensitivity. Reflecting differences in simulation between the data taking periods, as described in Sec. III, a single training is used for 2017, 2018A, and 2018B, with a separate training used for 2016 . The $S_{\mathrm{NN}}$ distributions for the simulated background, several signal models, and the 2016 and $2017+2018$ data are shown in Fig. 2.

For each of the six $N_{\text {jets }}$ bins, events are divided into four $S_{\mathrm{NN}}$ bins: $S_{\mathrm{NN}, 1}$ (lowest $S_{\mathrm{NN}}$ ), .., $S_{\mathrm{NN}, 4}$ (highest $S_{\mathrm{NN}}$ ). The $S_{\mathrm{NN}}$ bin boundaries are chosen separately for each $N_{\text {jets }}$ bin such that the expected significance for the $550 \mathrm{GeV}$ RPV signal model, which has expected significance close to 5 standard deviations $(\sigma)$, is maximized, under the constraint that the fraction of simulated $t \bar{t}$ events in each $S_{\mathrm{NN}}$ bin is the same for all $N_{\text {jets }}$ bins. For example, the fraction of all events in each $N_{\text {jets }}$ bin falling into the $S_{\mathrm{NN}, 1}$ bin is constrained to be approximately $56 \%$, while the fraction of events falling into the $S_{\mathrm{NN}, 4}$ bin is constrained to be approximately $2.4 \%$. This constraint removes the small dependence of $S_{\mathrm{NN}}$ on $N_{\text {jets }}$ that remains after NN training with gradient reversal.

In the maximum-likelihood fit, the $t \bar{t} N_{\text {jets }}$ distribution is parametrized with a function inspired by QCD jet scaling patterns [75] in which the ratio $R(i)=M_{i+1} / M_{i}$, where $M_{i}$ is the number of events with $N_{\text {jets }}=i$, can be described by a falling "Poisson" component at low $N_{\text {jets }}$ and a constant "staircase" component at high $N_{\text {jets }}$. This ratio is well modeled by the function,

$$
f(i)=a_{2}+\left[\frac{\left(a_{1}-a_{2}\right)^{i-7}}{\left(a_{0}-a_{2}\right)^{i-9}}\right]^{1 / 2} .
$$

Notice that $a_{0}=f(7), a_{1}=f(9)$, and $a_{2}$ is the asymptotic value for large $i$. This particular parametrization was chosen to avoid large correlations between the fit parameters. The $N_{\text {jets }}$ distribution for each $S_{\mathrm{NN}}$ bin $j$ (see Fig. 4) is modeled using a recursive expression given by $M_{i}^{j}=$ $Y_{7}^{j} \Pi_{k=7}^{i-1} f(k)$ where $Y_{7}^{j}$ are normalization parameters that are floating in the fit. The last $N_{\text {jets }}$ bin considered is an inclusive $N_{\text {jets }} \geq 12$ bin, such that $i \in[7,12]$. In the maximum-likelihood fit, the free parameters consist of the three shape parameters $a_{0}, a_{1}$, and $a_{2}$; the four normalization parameters $Y_{7}^{j}$; the signal strength; and all nuisance parameters related to systematic uncertainties described in Sec. V.
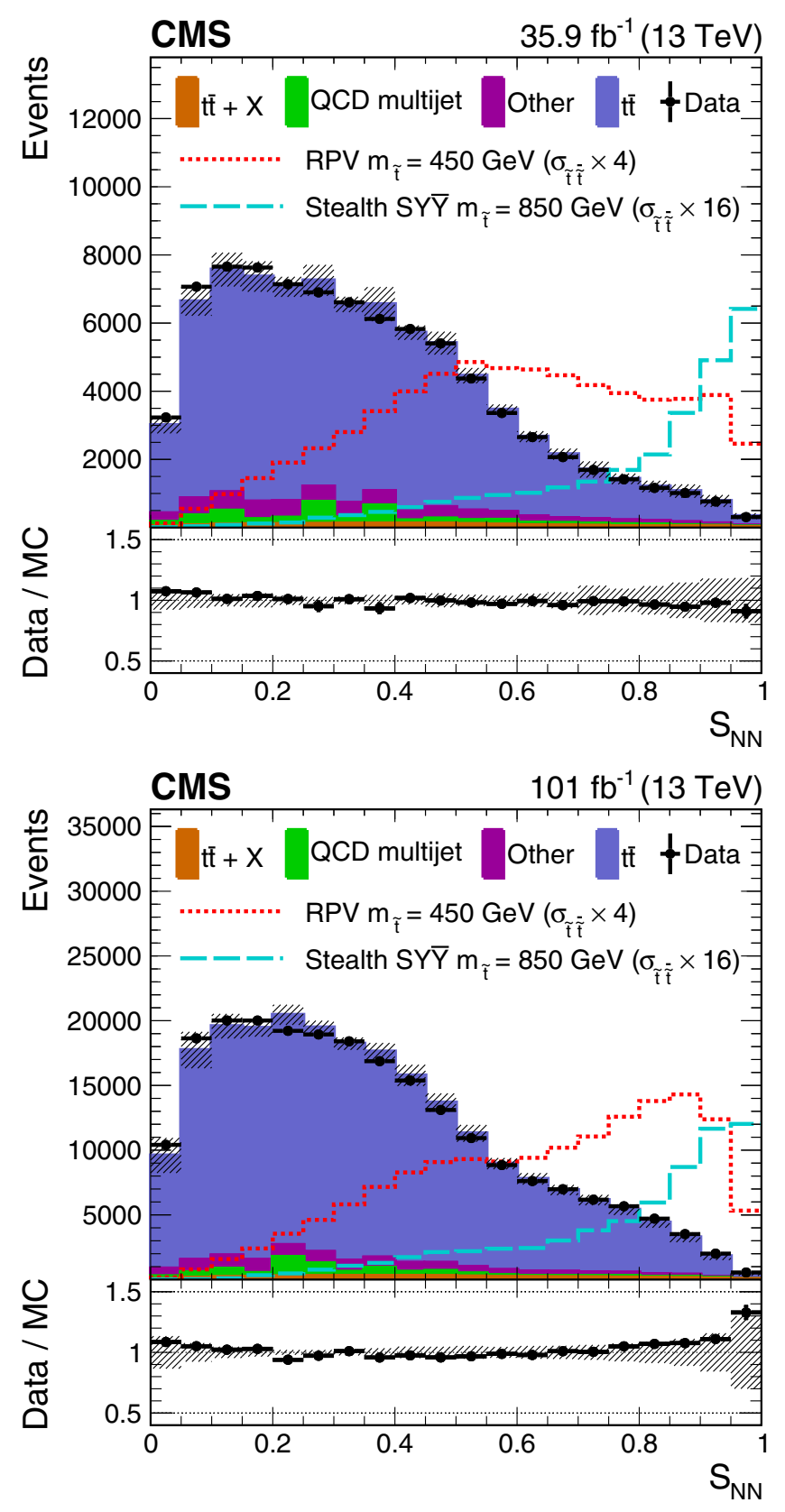

FIG. 2. The $S_{\mathrm{NN}}$ distributions for the 2016 training (upper) and $2017+2018$ training (lower) show the corresponding data in the SR (black points); simulated background normalized to the number of data events (filled histograms); RPV signal model with $m_{\tilde{t}}$ of $450 \mathrm{GeV}$ (red short dashed); and stealth $S Y \bar{Y}$ signal model with $m_{\tilde{t}}$ of $850 \mathrm{GeV}$ (cyan long dashed). All events shown pass the SR event selection. The band on the total background histogram denotes the dominant systematic uncertainties related to the modeling of $H_{\mathrm{T}}$, jet mass, and jet $p_{\mathrm{T}}$ in the $t \bar{t}$ simulation, as well as the statistical uncertainty for the non- $t \bar{t}$ components. The lower panel shows the ratio of the number of data events to the number of normalized simulated events with the band representing the difference between the nominal ratio and the ratio obtained when varying the total background by its uncertainty. 
The QCD background yield parameters are fixed in the fit at the values determined from the CR. More specifically, the QCD background prediction for each $N_{\text {jets }}-S_{\mathrm{NN}}$ bin in the $\mathrm{SR}$ is given by the yield for the same bin in the CR in data, after subtraction of the non-QCD backgrounds as predicted from simulation, multiplied by the ratio of SR to CR yields in simulation $\left(R_{\mathrm{QCD}}\right)$. This procedure is verified with a closure test in the simulation. The yield parameters from the minor backgrounds are also kept fixed in the fit at the values predicted by simulation. While the yield parameters are fixed in the fit, the ultimate contributions from QCD and minor backgrounds vary according to the constrained nuisance parameters related to systematic uncertainties in those fit components.

\section{SYSTEMATIC UNCERTAINTIES AND FIT VALIDATION}

As described in Sec. IV, an unbiased estimate of the dominant $t \bar{t}$ background is obtained from the fit to data as long as the $t \bar{t} N_{\text {jets }}$ shape is the same for all four $S_{\mathrm{NN}}$ bins. By construction, $N_{\text {jets }}$ shape invariance is achieved in the simulation with an $N_{\text {jets }}$-specific $S_{\mathrm{NN}}$ binning as described in the previous section. Thus, systematic uncertainties on the $t \bar{t}$ background are important to the degree that they violate the assumption that the $S_{\mathrm{NN}}$ binning determined in simulation also applies to the data. We quantify how each source of uncertainty causes deviations from the assumed $N_{\text {jets }}$ shape invariance by comparing the nominal $N_{\text {jets }}$ shape to the $N_{\text {jets }}$ shapes in all $S_{\mathrm{NN}}$ bins after performing the relevant systematic variation to the $t \bar{t}$ simulation. Each systematic variation is associated with a constrained nuisance parameter in the fit. The deviation in shape for each $N_{\text {jets }}$ distribution, derived from the ratio of the postvariation shape divided by the nominal shape, changes linearly with the associated nuisance parameter for the systematic variation, while preserving the normalization of the distribution.

Sources of $t \bar{t}$ shape uncertainty include uncertainty in aspects of event generation including PDFs, choice of renormalization and factorization scales $\left(\mu_{\mathrm{R}}, \mu_{\mathrm{F}}\right.$ scales), and parton shower modeling, which is itself composed of aspects related to modeling of ISR, FSR, color reconnection in the parton shower, matrix element-parton shower matching scale (ME-PS), underlying event (UE tune), and pileup modeling. The uncertainty due to the choice in $\left(\mu_{\mathrm{R}}\right.$, $\mu_{\mathrm{F}}$ ) scales is determined by independently varying both by factors of 2.0 and 0.5 excluding the variations $(2.0,0.5)$ and $(0.5,2.0)[55,76,77]$. The ISR and FSR uncertainties originate from variations of the renormalization scale for the parton shower by factors 0.5 and 2.0, effectively varying the value of $\alpha_{\mathrm{S}}$. The color reconnection uncertainty is calculated by allowing resonant decays to occur before the merging of multi-parton systems. The ME-PS uncertainty is obtained by varying the POWHEG parameter that governs ME-PS matching about its nominal value according to $h_{\text {damp }}=1.379_{-0.505}^{+0.926}$ times the top quark mass [63]. The UE tune uncertainty comes from variation of the PYTHIA parameters that control the modeling of the underlying event as described in Ref. [63]. The total inelastic $p p$ cross section is changed by $5 \%$ to estimate the uncertainty related to pileup [78].

Sources of $t \bar{t}$ shape uncertainty related mostly to aspects of detector simulation include determination of jet energy scale (JES) and resolution (JER), modeling of the $b$ tagging efficiency, modeling of the efficiency for lepton triggers, identification, and isolation (lepton efficiencies); residual mismodeling of $H_{\mathrm{T}}$, jet $p_{\mathrm{T}}$, and jet mass; and use of the CR for measuring deviations from the assumption of $N_{\text {jets }}$ shape invariance.

The uncertainty in the modeling of $H_{\mathrm{T}}$ in the $t \bar{t}$ simulation is composed of four separate components. The first $H_{\mathrm{T}}$ uncertainty (primary) is taken as the full difference in the $t \bar{t}$ background shape with and without the $H_{\mathrm{T}}$ correction. The second $H_{\mathrm{T}}$ uncertainty (validation) is taken as the difference between the simulation with nominal $H_{\mathrm{T}}$ correction (described in Sec. III) and the observed $H_{\mathrm{T}}$ distribution in the signal-depleted SR sample with $N_{\text {jets }}=8$. The third and fourth $H_{\mathrm{T}}$ uncertainties address the choices of parameterization of the $H_{\mathrm{T}}$ correction as functions of $H_{\mathrm{T}}$ and $N_{\text {jets. }}$. For these, we take the uncertainty as the difference between the nominal correction and two alternate corrections that use the $H_{\mathrm{T}}=$ $2000 \mathrm{GeV}$ correction for all events with $H_{\mathrm{T}}>2000 \mathrm{GeV}$ ( $H_{\mathrm{T}}$-parametrization) and the $N_{\text {jets }}=7$ correction for all values of $N_{\text {jets }}\left(N_{\text {jets }}\right.$-parametrization).

Comparisons of data and simulation in the CR show that the simulation predicts distributions with higher values of jet $p_{\mathrm{T}}$ and mass than observed. The observed discrepancy at jet $p_{\mathrm{T}}$ (mass) of $400(50) \mathrm{GeV}$ depends on jet $p_{\mathrm{T}}$ rank and is small for the highest $p_{\mathrm{T}}$ jet in each event growing to approximately $50 \%$ for the jet with sixth-highest $p_{\mathrm{T}}$ in each event. Similar trends are observed in the signal-depleted, $t \bar{t}$-dominated SR with $N_{\text {jets }}=7$. In the CR, the discrepancy in the falling tail of each distribution is minimized when the $p_{\mathrm{T}}$ (mass) of each jet is scaled by the value $0.95,0.95,0.95$, $0.95(0.95,1.01,0.98,0.98)$ for $2016,2017,2018 \mathrm{~A}$, and $2018 \mathrm{~B}$, respectively. Thus, the related $t \bar{t}$ shape uncertainty is taken to be the resulting difference between scaled and nominal simulated $t \bar{t}$ distributions. The dependence on jet $p_{\mathrm{T}}$ rank indicates that the discrepancy arises predominantly in the event generation; however, we choose to estimate the associated systematic uncertainty separately for each data taking period to include potential effects of detector response simulation. The $H_{\mathrm{T}}$ correction is omitted from the determination of these jet $p_{\mathrm{T}}$ and mass uncertainties to avoid double counting of $H_{\mathrm{T}}$ mismodeling effects. In addition, because the estimation of jet $p_{\mathrm{T}}$ and mass uncertainties relies on variable scaling (rather than event reweighting), the uncertainties include effects of 
changes in the $S_{\mathrm{NN}}$ for each event, which is not included in the $H_{\mathrm{T}}$ uncertainty.

As mentioned above, the use of $N_{\text {jets }}$-dependent $S_{\mathrm{NN}}$ binning ensures that the $N_{\text {jets }}$ shape is the same in all four $S_{\mathrm{NN}}$ bins in simulation, and the use of the same binning in the data assumes that the $N_{\text {jets }}-S_{\mathrm{NN}}$ dependence is well modeled in the simulation. This assumption is confirmed and a related systematic uncertainty is determined by comparing the $N_{\text {jets }}$ shapes (in five uniform $S_{\mathrm{NN}}$ bins) for data and simulation in the CR. For each of the six $N_{\text {jets }}$ bins, we compute the ratio $R_{M}=\left(1 / \mu_{i}\right)\left(M_{\text {all }} / M_{i}\right)$ as a
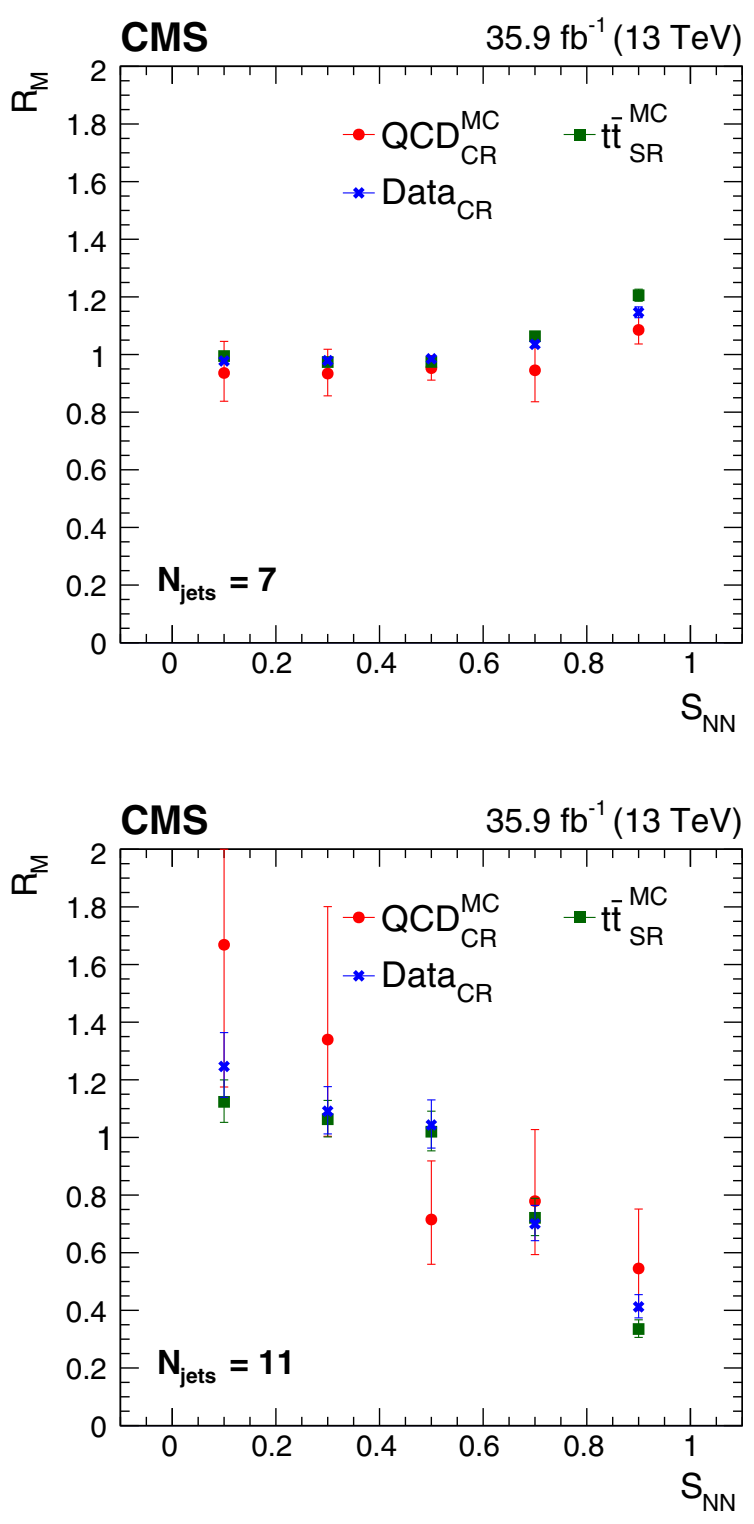

FIG. 3. Distribution in $S_{\mathrm{NN}}$ of the ratio $R_{M}$, as defined in the text, for $N_{\text {jets }}=7$ (upper) and 11 (lower), for the QCD CR simulation (red circles), the $t \bar{t} \mathrm{SR}$ simulation (green squares), and data in the CR (blue crosses) for the 2016 data period. The error bars indicate the statistical uncertainty in the value of $R_{M}$. function of $S_{\mathrm{NN}}$, where $M_{\text {all }}$ is the total number of events in all $N_{\text {jets }}$ bins, $M_{i}$ is the total number of events in the $N_{\text {jets }}=i$ bin, and $\mu_{i}$ is the uncertainty-weighted average of $M_{\text {all }} / M_{i}$ in the $N_{\text {jets }}=i$ bin used to facilitate comparison of the $R_{M}$ shapes between samples and $N_{\text {jets }}$ bins with different normalizations. Figure 3 shows a comparison of $R_{M}$ (from $N_{\text {jets }}=7$ and 11 in the 2016 analysis) for simulation of the QCD background in the $\mathrm{CR}\left(\mathrm{QCD}_{\mathrm{CR}}^{\mathrm{MC}}\right)$, simulation of $t \bar{t}$ in the $\mathrm{SR}\left(t \bar{t}_{\mathrm{SR}}^{\mathrm{MC}}\right)$, and the data in the QCD background-dominated $\mathrm{CR}\left(\mathrm{Data}_{\mathrm{CR}}\right)$. Agreement between $\mathrm{QCD} \mathrm{CR}_{\mathrm{MC}}$ and $t \bar{t}_{\mathrm{SR}}^{\mathrm{MC}}$ demonstrates that QCD backgrounddominated data in the $\mathrm{CR}$ are a good proxy for $t \bar{t}$ dominated data in the SR, and agreement between $\mathrm{QCD}_{\mathrm{CR}}^{\mathrm{MC}}$ and $\mathrm{Data}_{\mathrm{CR}}$ verifies that the dependence of the $N_{\text {jets }}$ shape on $S_{\mathrm{NN}}$ is well modeled in the simulation. Similar agreement is found for the $R_{M}$ distributions for the other $N_{\text {jets }}$ bins and data periods. The uncertainty related to the combination of both effects is taken as the difference between $t \bar{t}_{\mathrm{SR}}^{\mathrm{MC}}$ and $\operatorname{Data}_{\mathrm{CR}}$.

TABLE I. Summary of the impact of systematic uncertainties in the expected event yields for the $t \bar{t}$ background, minor backgrounds (both $t \bar{t}+X$ and other), and the RPV signal model with $m_{\tilde{t}}=550 \mathrm{GeV}$. Abbreviated names for each source of uncertainty are explained in the text. For sources of uncertainty for which the size of the impact depends on $N_{\text {jets }}$ and $S_{\mathrm{NN}}$, a representative range of values showing the 16th and 84th percentile of all the corrections is listed with the maximum value from all bins shown in parentheses. All values are in units of percent.

\begin{tabular}{lccc}
\hline \hline $\begin{array}{l}\text { Source of } \\
\text { uncertainty }\end{array}$ & $\begin{array}{c}\bar{t} \\
\text { background }\end{array}$ & $\begin{array}{c}\text { Minor } \\
\text { background }\end{array}$ & $\begin{array}{c}\text { RPV } \\
\text { signal }\end{array}$ \\
\hline PDFs & $0-1(2)$ & $0-1(8)$ & $0-2(7)$ \\
$\left(\mu_{\mathrm{R}}, \mu_{\mathrm{F}}\right)$ scales & $0-2(5)$ & $1-8(18)$ & $0-3(4)$ \\
ISR & $0-4(15)$ & $\ldots$ & $\ldots$ \\
FSR & $0-8(27)$ & $\ldots$ & $\ldots$ \\
Color reconnection & $0-10(44)$ & $\ldots$ & $\ldots$ \\
ME-PS & $0-14(82)$ & $\ldots$ & $\ldots$ \\
UE tune & $0-7(100)$ & $\ldots$ & $\ldots$ \\
Pileup & $0-2(7)$ & $0-7(28)$ & $0-2(4)$ \\
JES & $0-4(18)$ & $5-21(100)$ & $1-11(31)$ \\
JER & $0-2(10)$ & $1-15(100)$ & $0-6(14)$ \\
$b$ tagging & $0-1(3)$ & $0-2(12)$ & $0-2(2)$ \\
Lepton efficiencies & $0-1(1)$ & $3-5(5)$ & $3-4(4)$ \\
$H_{\mathrm{T}}$ primary & $0-5(17)$ & $\ldots$ & $\ldots$ \\
$H_{\mathrm{T}}$ validation & $0-1(4)$ & $0-6(10)$ & $\ldots$ \\
$H_{\mathrm{T}} H_{\mathrm{T}}{ }^{-}$ & $0-2(9)$ & $\ldots$ & $\ldots$ \\
$\quad$ parametrization & & & $\ldots$ \\
$H_{\mathrm{T}} N_{\text {jets }}{ }^{-}$ & $0-7(27)$ & $\ldots$ & $\ldots$ \\
$\quad$ parametrization & & & $\ldots$ \\
Jet $p_{\mathrm{T}}$ & $0-4(15)$ & $\ldots$ & $\ldots$ \\
Jet mass & $0-4(15)$ & $\ldots$ & $\ldots$ \\
$N_{\text {jets }}$ shape invariance & $0-12(37)$ & $\ldots$ & $\ldots$ \\
Integrated luminosity & $\ldots$ & $2.3-2.5$ & $2.3-2.5$ \\
Theoretical cross & $\ldots$ & 30 & $\ldots$ \\
$\quad$ section & & & \\
\hline \hline & & & $\ldots$ \\
\hline
\end{tabular}



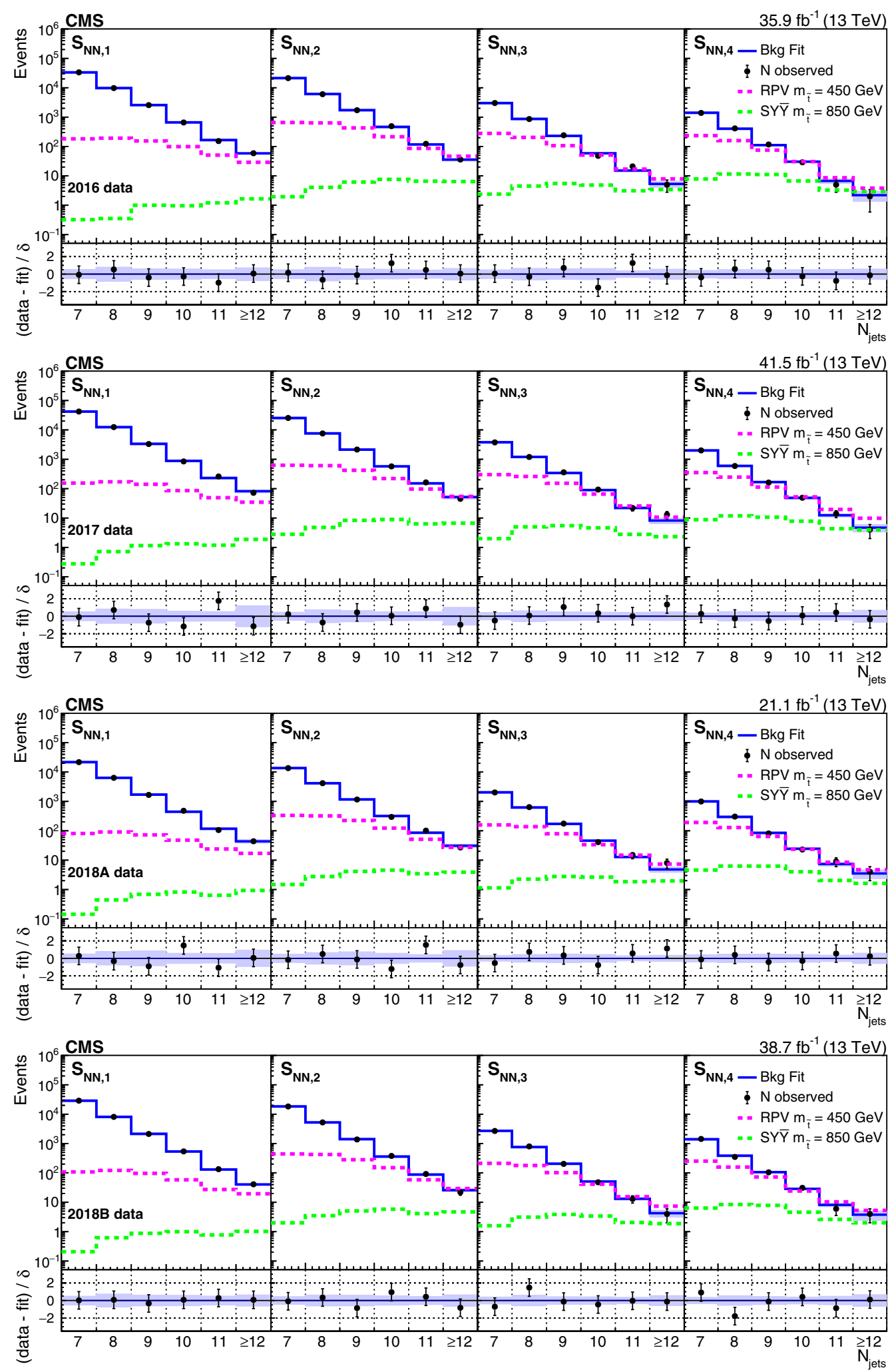

FIG. 4. Fitted background prediction and observed data counts for 2016, 2017, 2018A, and 2018B (from upper to lower rows) as functions of $N_{\text {jets }}$ in each of the four bins in $S_{\mathrm{NN}}$. The signal distributions normalized to the predicted cross section for the RPV model with $m_{\tilde{t}}=450 \mathrm{GeV}$ and the stealth $S Y \bar{Y}$ model with $m_{\tilde{t}}=850 \mathrm{GeV}$ are shown for comparison. The lower panel of each plot displays the difference between the number of observed events and the number of events determined by the fit divided by the statistical uncertainty associated with the observed number of events $(\delta)$ as black points with error bars denoting $\delta$. The blue band shows the total systematic uncertainty in the fit from all nuisance parameters. 
For the QCD background, the shape is obtained from data in the CR, and the normalization is set with $R_{\mathrm{QCD}}$. Because the systematic uncertainties in the simulation largely cancel in the $R_{\mathrm{QCD}}$ ratio, the uncertainty in $R_{\mathrm{QCD}}$ is dominated by the statistical uncertainty of simulated samples and ranges from 15\%-25\% depending on data collection period.

Sources of systematic uncertainty in the predictions for signals and the minor backgrounds include PDFs, JES, JER, $b$ tagging efficiency, lepton efficiency, trigger efficiency, $\left(\mu_{\mathrm{R}}, \mu_{\mathrm{F}}\right)$ scales, cross sections for the minor backgrounds, and a $2.3 \%-2.5 \%$ uncertainty in the integrated luminosity [79-81]. Since the signal and minor backgrounds are estimated directly from simulation, related uncertainties are included as the full effect of the systematic variation on the yields in each $N_{\text {jets }}$ and $S_{\mathrm{NN}}$ bin, thereby taking into account normalization effects as well as shape changes.

Uncertainties derived from comparisons of data and simulation separately in each data taking period (related to pileup, JES, JER, $b$ tagging efficiency, lepton efficiencies, $H_{\mathrm{T}}$ corrections, $N_{\text {jets }}$ shape invariance, and integrated luminosity) are treated as uncorrelated among all data samples. Uncertainties related to parton shower modeling are treated as fully correlated for 2017, 2018A, and 2018B, while the corresponding uncertainties for 2016 are

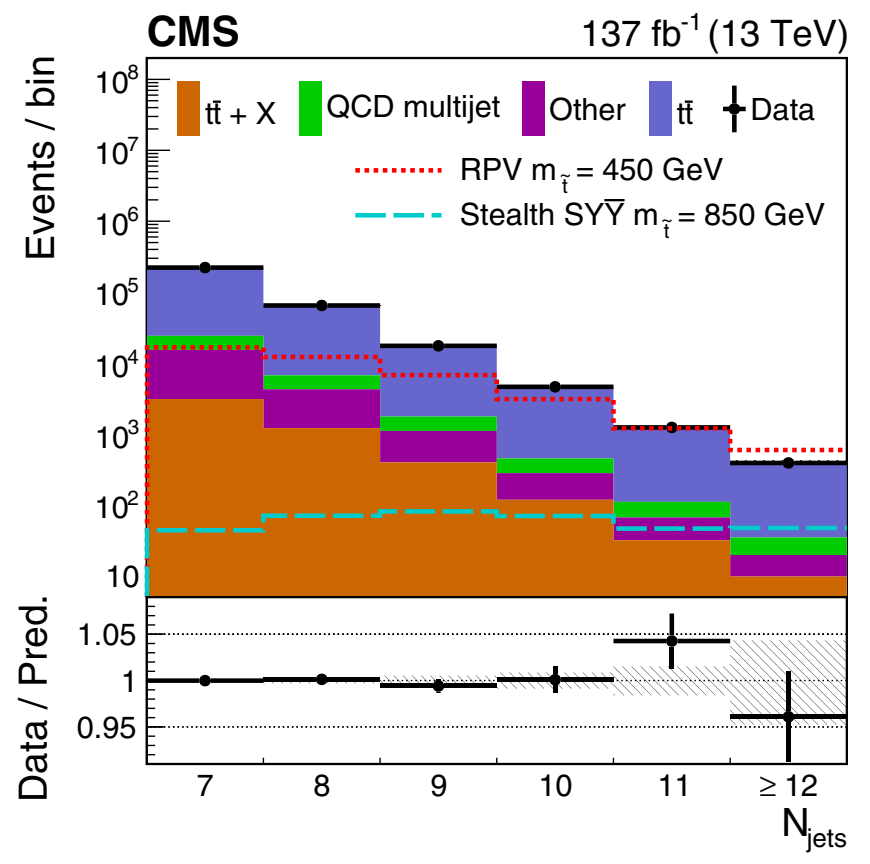

FIG. 5. Background prediction from the background-only fit and observed data counts as a function of $N_{\text {jets }}$ summed over data periods and $S_{\mathrm{NN}}$ bins. Overlaid are expected distributions for the RPV and stealth $S Y \bar{Y}$ models with $m_{\tilde{t}}=450$ and $850 \mathrm{GeV}$, respectively, normalized according to the top squark pair production cross section. For visualization purposes, the hatched band in the lower panel shows the quadrature sum of all of the uncertainties on the background prediction. uncorrelated with those from the other data taking periods; uncertainties related to $\left(\mu_{\mathrm{R}}, \mu_{\mathrm{F}}\right)$ scales and cross sections for the minor backgrounds are treated as correlated between all four periods.

Table I shows the impact of the systematic uncertainties on the expected event yields for the $t \bar{t}$ background, minor backgrounds, and the RPV signal model with $m_{\tilde{t}}=550 \mathrm{GeV}$. For sources of uncertainty for which the size of the impact depends on $N_{\text {jets }}$ and $S_{\mathrm{NN}}$, a representative range of values is listed along with the maximum value from all bins.

\section{RESULTS AND INTERPRETATION}

The results of the fit to 2016, 2017, 2018A, and 2018B datasets with the signal strength fixed to zero (backgroundonly fit) are shown along with the observed number of events in Fig. 4; each column (row) in the plot array
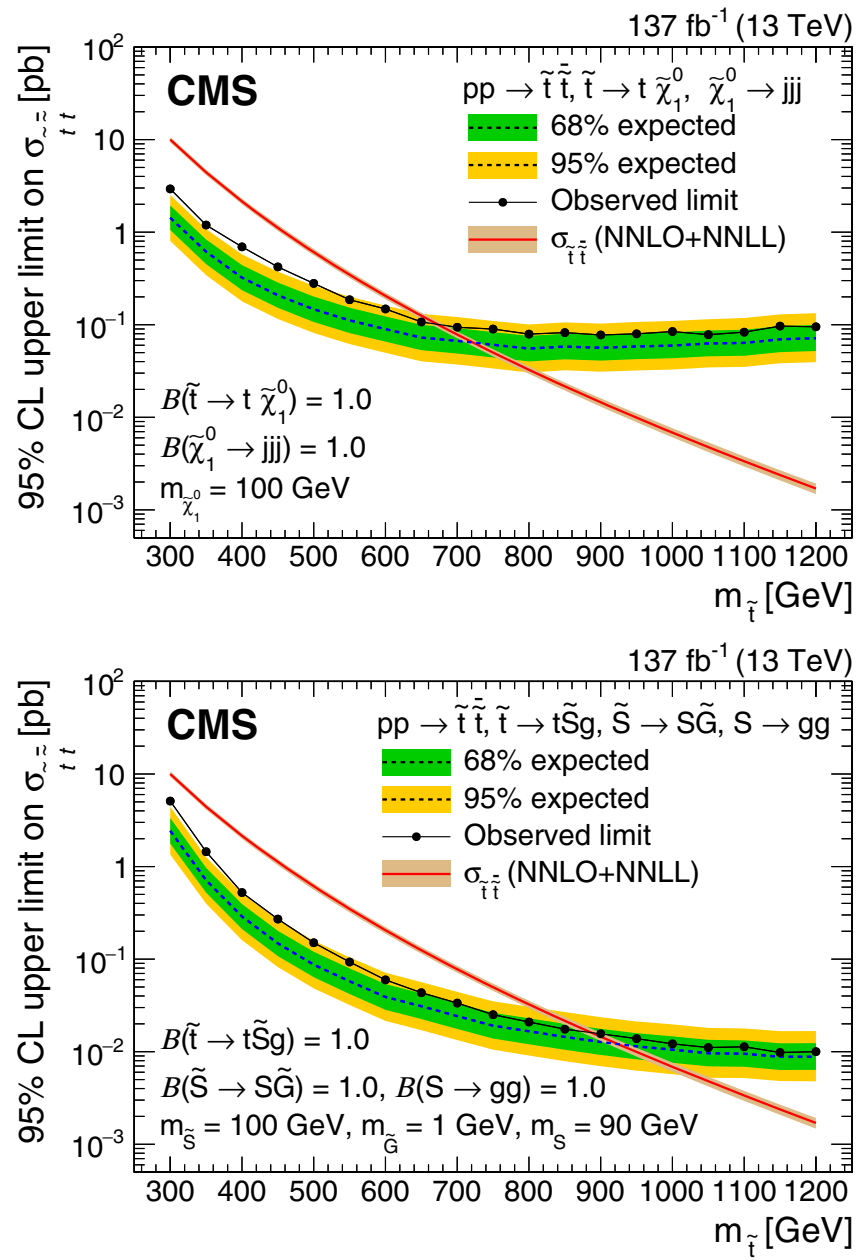

FIG. 6. Expected and observed 95\% CL upper limit on the top squark pair production cross section as a function of the top squark mass for the RPV (upper) and stealth $S Y \bar{Y}$ (lower) SUSY models. Particle masses and branching fractions assumed for each model are included on each plot. The expected cross section computed at NNLO + NNLL accuracy is shown in the red curve. 
corresponds to a specific $S_{\mathrm{NN}}$ bin (dataset). The expected distributions for top squark pair production in the specific RPV $\left(m_{\tilde{t}}=450 \mathrm{GeV}\right)$ and stealth $S Y \bar{Y}$ models $\left(m_{\tilde{t}}=850 \mathrm{GeV}\right)$ described in Sec. I are overlaid for illustration purposes. The lower panel of each plot displays the difference between the observed number of events and the total number of expected events determined by the fit divided by the statistical uncertainty associated with the observed number of events $(\delta)$ as black points with error bars denoting $\delta$. The blue band shows the total uncertainty in the fit determined from the full fit covariance matrix in order to account for the correlations among fit parameters. Figure 5 shows the results of the same background-only fit summed over $S_{\mathrm{NN}}$ bins and data periods with separate contributions from each background.

The data are also used to determine the $95 \%$ confidence level (CL) upper limits on $\sigma_{\tilde{t} \bar{t}}$ and the signal strength $p$-values [82] for both the RPV and stealth $S Y \bar{Y}$ models obtained using the $\mathrm{CL}_{\mathrm{s}}$ approach [83-85] with asymptotic formulas [86] and the profile likelihood ratio as the test statistic. Figure 6 shows the expected and observed cross section limits as a function of $m_{\tilde{t}}$ for the benchmark RPV and stealth $S Y \bar{Y}$ signal models. Comparing to the predicted cross section, these limits correspond to the exclusion of top squark masses in the range $300-670$ and $300-870 \mathrm{GeV}$ for the benchmark RPV and stealth $S Y \bar{Y}$ models, respectively. Figure 7 shows the local $p$-value [82] of the signal strength, as a function of $m_{\tilde{t}}$, obtained from fits to the data with each signal strength as a free parameter for both the RPV and stealth $S Y \bar{Y}$ models. The $p$-value quantifies the probability for the background to produce an upward fluctuation at least as large as that observed. Fits are performed and $p$-values obtained separately for each dataset, as well as in a simultaneous fit to all datasets. We observe the most extreme $p$-value to be 0.003 , which corresponds to a local significance of $2.8 \sigma$ and a best fit signal strength of $0.21 \pm 0.07$ for the RPV model with $m_{\tilde{t}}=400 \mathrm{GeV}$ assuming unity branching fractions for the decays described in Sec. I.

The $2.8 \sigma$ local significance for the RPV model with $m_{\tilde{t}}=400 \mathrm{GeV}$ is understood to arise from a combination of two effects. First, although the level of agreement between the observed data and the background expectation shown in Fig. 4 is reasonable, the agreement improves when the signal is included in the fit, contributing approximately $1.1 \sigma$ to the significance. Second, the constrained nuisance parameters (NP) are pulled less from their initial values when the signal is included in the fit, contributing approximately $1.7 \sigma$ to the significance. This second effect is illustrated in Fig. 8 which shows for each of a selection of NP: the fit value $(\theta)$ and uncertainty $\left(\delta_{\theta}\right)$ from both the background-only fit (b) and the signal + background fit $(\mathrm{s}+\mathrm{b})$, as well as the $\Delta \chi^{2} \equiv \chi^{2}(\mathrm{~s}+\mathrm{b})-$ $\chi^{2}$ (b) difference of $\chi^{2} \equiv\left(\theta / \delta_{\theta}\right)^{2}$ from the two fits. A $\theta$ value of one indicates that the fit value of the nuisance parameter is 1 standard deviation from its nominal value, and a $\delta_{\theta}$ value less than one shows that the uncertainty is
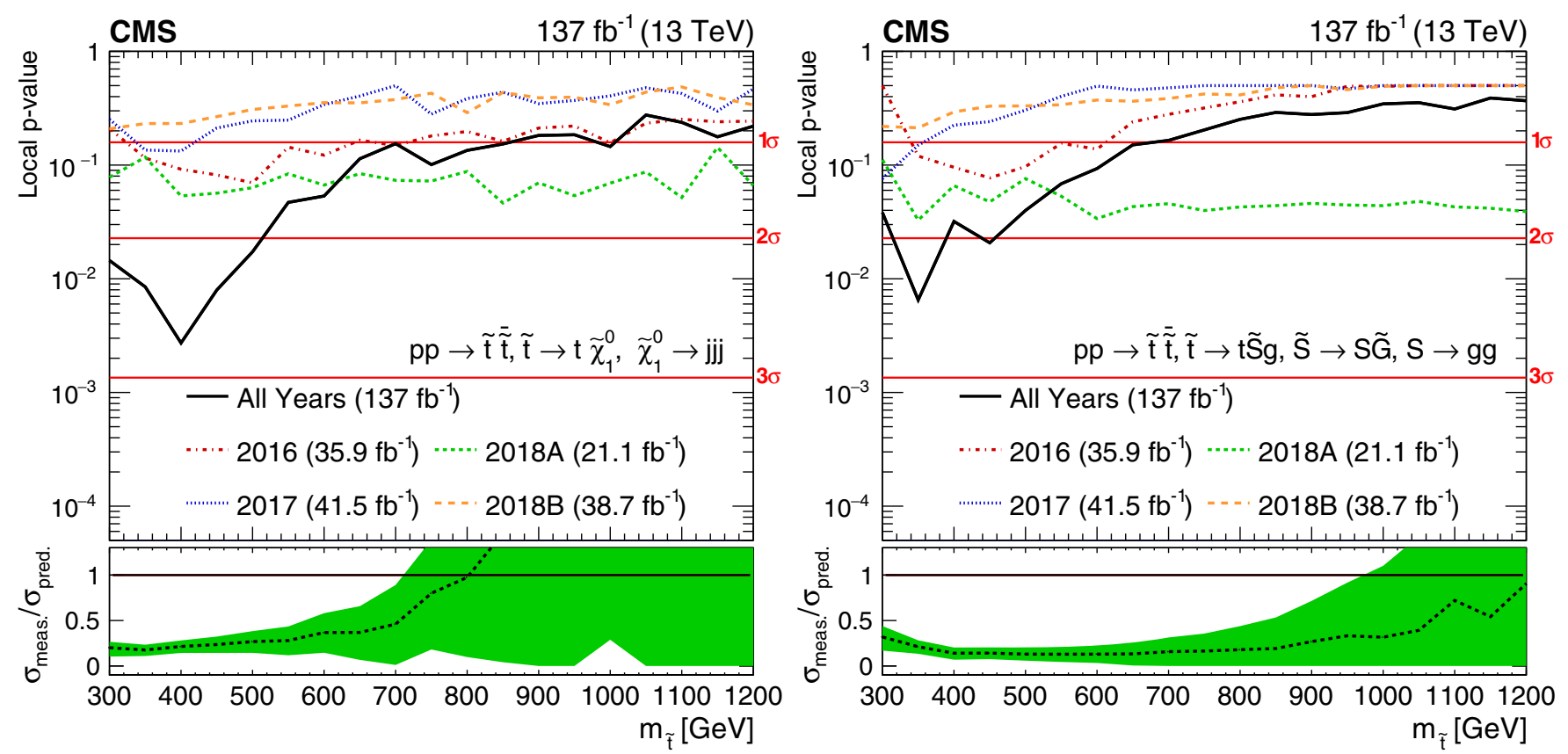

FIG. 7. Local $p$-value as a function of top squark mass for the RPV (left) and stealth $S Y \bar{Y}$ models (right). The colored lines show the $p$-values for separate fits of the 2016 (red dash dotted), 2017 (blue dotted), 2018A (green short dashed), and 2018B (orange long dashed) datasets; the black line shows the $p$-value for the simultaneous fit of datasets. The lower panels show the best fit signal strength $\left(\sigma_{\text {meas }} / \sigma_{\text {pred }}\right)$ as a function of top squark mass with uncertainty denoted by the green band. 


\section{CMS}

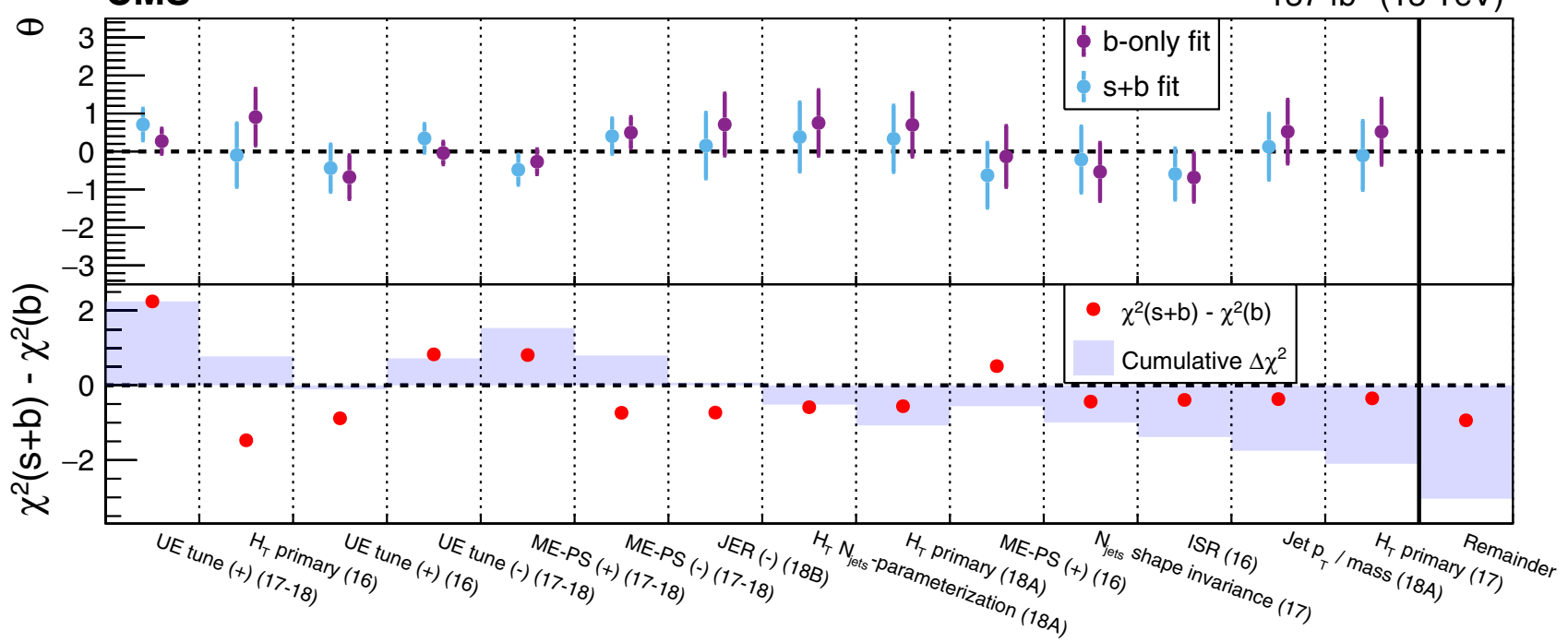

FIG. 8. The upper panel shows the fit values $(\theta)$ and uncertainties $\left(\delta_{\theta}\right)$ for a selection of nuisance parameters (NP) from both the background-only fit (purple) and signal + background fit (blue) for the RPV model with $m_{\tilde{t}}=400 \mathrm{GeV}$. The $\mathrm{x}$-axis labels refer to the NP sources described in Sec. V, the data period $(16,17$, etc.), and the direction of variation $(+,-)$. The lower panel shows the $\Delta \chi^{2} \equiv \chi^{2}(\mathrm{~s}+\mathrm{b})-\chi^{2}(\mathrm{~b})$ difference of $\chi^{2} \equiv\left(\theta / \delta_{\theta}\right)^{2}$ from the signal + background $(\mathrm{s}+\mathrm{b})$ and background-only (b) fits as a red point for each NP and the cumulative sum of $\Delta \chi^{2}$ from left to right as a blue shaded histogram. The fourteen selected NP are those with $\left|\Delta \chi^{2}\right|>0.3$, and the NP are ordered from left to right by decreasing $\left|\Delta \chi^{2}\right|$. The rightmost bin, separated by a vertical solid line, shows the sum of $\Delta \chi^{2}$ for all NP not displayed in the figure (red point) and the sum of $\Delta \chi^{2}$ for all NP (blue shaded histogram).

reduced in the fit relative to its initial value. All NPs have $\theta$ values below one for the background-only fit, and several NPs related to $t \bar{t}$ modeling are constrained with $\delta_{\theta}$ in the range of $0.25-0.40$. Figure 8 also shows the cumulative and total sums of $\Delta \chi^{2}$ for the NPs, with the sum for all NP of $\sum \Delta \chi^{2}=-3.0$ corresponding to an approximate contribution to the signal significance of $\sqrt{\left|\sum \Delta \chi^{2}\right|}=1.7 \sigma$.

\section{SUMMARY}

A first of its kind search for top squark pair production with subsequent decay characterized by two top quarks, additional gluons or light-flavor quarks, and low missing transverse momentum $\left(p_{\mathrm{T}}^{\text {miss }}\right)$ is described. Events containing exactly one electron or muon and at least seven jets, of which at least one should be $b$ tagged, are selected from a sample of proton-proton collisions at $\sqrt{s}=13 \mathrm{TeV}$ corresponding to an integrated luminosity of $137 \mathrm{fb}^{-1}$ collected with the CMS detector in 2016-2018. No requirement is made on $p_{\mathrm{T}}^{\text {miss }}$. The dominant $t \bar{t}$ background is predicted from data using a simultaneous fit of the jet multiplicity distribution across four bins of a neural network score.

The results are interpreted in terms of top squark pair production in the context of $R$-parity violating (RPV) and stealth supersymmetry models. Top squark masses $\left(m_{\tilde{t}}\right)$ up to $670 \mathrm{GeV}$ are excluded at $95 \%$ confidence level for the RPV model in which the top squark decays to a top quark and the lightest neutralino, which subsequently decays to three light-flavor quarks via an off shell squark through a trilinear coupling $\lambda^{\prime \prime}$. Top squark masses up to $870 \mathrm{GeV}$ are excluded for the stealth supersymmetry model in which the top squark decays to a top quark, three gluons, and a gravitino via intermediate hidden sector particles. The maximum observed local significance is 2.8 standard deviations corresponding to a best fit signal strength of $0.21 \pm 0.07$ for the RPV model with $m_{\tilde{t}}=400 \mathrm{GeV}$.

\section{ACKNOWLEDGMENTS}

We congratulate our colleagues in the CERN accelerator departments for the excellent performance of the LHC and thank the technical and administrative staffs at CERN and at other CMS institutes for their contributions to the success of the CMS effort. In addition, we gratefully acknowledge the computing centers and personnel of the Worldwide LHC Computing Grid and other centers for delivering so effectively the computing infrastructure essential to our analyses. Finally, we acknowledge the enduring support for the construction and operation of the LHC, the CMS detector, and the supporting computing infrastructure provided by the following funding agencies: BMBWF and FWF (Austria); FNRS and FWO (Belgium); CNPq, CAPES, FAPERJ, FAPERGS, and FAPESP (Brazil); MES (Bulgaria); CERN; CAS, MoST, and NSFC (China); COLCIENCIAS (Colombia); MSES and CSF (Croatia); RIF (Cyprus); SENESCYT (Ecuador); MoER, ERC PUT and ERDF (Estonia); Academy of Finland, MEC, and HIP 
(Finland); CEA and CNRS/IN2P3 (France); BMBF, DFG, and HGF (Germany); GSRT (Greece); NKFIA (Hungary); DAE and DST (India); IPM (Iran); SFI (Ireland); INFN (Italy); MSIP and NRF (Republic of Korea); MES (Latvia); LAS (Lithuania); MOE and UM (Malaysia); BUAP, CINVESTAV, CONACYT, LNS, SEP, and UASLP-FAI (Mexico); MOS (Montenegro); MBIE (New Zealand); PAEC (Pakistan); MSHE and NSC (Poland); FCT (Portugal); JINR (Dubna); MON, RosAtom, RAS, RFBR, and NRC KI (Russia); MESTD (Serbia); SEIDI, CPAN, PCTI, and FEDER (Spain); MOSTR (Sri Lanka); Swiss Funding Agencies (Switzerland); MST (Taipei); ThEPCenter, IPST, STAR, and NSTDA (Thailand); TUBITAK and TAEK (Turkey); NASU (Ukraine); STFC (United Kingdom); DOE and NSF (USA). Individuals have received support from the Marie-Curie program and the European Research Council and Horizon 2020 Grant, Contracts No. 675440, No. 724704, No. 752730, and No. 765710 (European Union); the Leventis Foundation; the Alfred P. Sloan Foundation; the Alexander von Humboldt Foundation; the Belgian Federal Science Policy Office; the Fonds pour la Formation à la Recherche dans l'Industrie et dans l'Agriculture (FRIA-Belgium); the Agentschap voor Innovatie door Wetenschap en Technologie (IWT-Belgium); the F. R. S.-FNRS and FWO (Belgium) under the "Excellence of Science-EOS"-be.h Project No. 30820817; the Beijing Municipal Science and Technology Commission, No. Z191100007219010; the Ministry of Education, Youth and Sports (MEYS) of the Czech Republic; the Deutsche Forschungsgemeinschaft (DFG), under Germany's Excellence Strategy-EXC
2121 "Quantum Universe"-390833306, and under Project No. 400140256-GRK2497; the Lendület ("Momentum") Program and the János Bolyai Research Scholarship of the Hungarian Academy of Sciences, the New National Excellence Program ÚNKP, the NKFIA Research Grants No. 123842, No. 123959, No. 124845, No. 124850 , No. 125105 , No. 128713 , No. 128786 , and No. 129058 (Hungary); the Council of Science and Industrial Research, India; the HOMING PLUS program of the Foundation for Polish Science, cofinanced from European Union, Regional Development Fund, the Mobility Plus program of the Ministry of Science and Higher Education, the National Science Center (Poland), Contracts Harmonia No. 2014/14/M/ST2/00428, Opus No. 2014/13/B/ST2/02543, No. 2014/15/B/ST2/03998, and No. 2015/19/B/ST2/02861, Sonata-bis No. 2012/07/ E/ST2/01406; the National Priorities Research Program by Qatar National Research Fund; the Ministry of Science and Higher Education, Project No. 0723-2020-0041 (Russia); the Programa Estatal de Fomento de la Investigación Científica y Técnica de Excelencia María de Maeztu, Grant No. MDM-2015-0509 and the Programa Severo Ochoa del Principado de Asturias; the Thalis and Aristeia programs cofinanced by EU-ESF and the Greek NSRF; the Rachadapisek Sompot Fund for Postdoctoral Fellowship, Chulalongkorn University and the Chulalongkorn Academic into Its 2nd Century Project Advancement Project (Thailand); the Kavli Foundation; the Nvidia Corporation; the SuperMicro Corporation; the Welch Foundation, Contract No. C-1845; and the Weston Havens Foundation (USA).
[1] P. Fayet and S. Ferrara, Supersymmetry, Phys. Rep. 32, 249 (1977).

[2] S. P. Martin, A supersymmetry primer, Adv. Ser. Dir. High Energy Phys. 21, 1 (1997).

[3] S. Dimopoulos and G. F. Giudice, Naturalness constraints in supersymmetric theories with nonuniversal soft terms, Phys. Lett. B 357, 573 (1995).

[4] R. Barbieri and G. F. Giudice, Upper bounds on supersymmetric particle masses, Nucl. Phys. B306, 63 (1988).

[5] A. Pomarol and D. Tommasini, Horizontal symmetries for the supersymmetric flavor problem, Nucl. Phys. B466, 3 (1996).

[6] A. G. Cohen, D. B. Kaplan, and A. E. Nelson, The more minimal supersymmetric standard model, Phys. Lett. B 388, 588 (1996).

[7] M. Papucci, J. T. Ruderman, and A. Weiler, Natural SUSY endures, J. High Energy Phys. 09 (2012) 035.

[8] C. Brust, A. Katz, S. Lawrence, and R. Sundrum, SUSY, the third generation and the LHC, J. High Energy Phys. 03 (2012) 103.
[9] R. Barbier, C. Berat, M. Besancon, M. Chemtob, A. Deandrea, E. Dudas, P. Fayet, S. Lavignac, G. Moreau, E. Perez, and Y. Sirois, $R$-parity violating supersymmetry, Phys. Rep. 420, 1 (2005).

[10] D. S. M. Alves, E. Izaguirre, and J. G. Wacker, Where the sidewalk ends: Jets and missing energy search strategies for the 7 TeV LHC, J. High Energy Phys. 10 (2011) 012.

[11] M. Lisanti, P. Schuster, M. Strassler, and N. Toro, Study of LHC searches for a lepton and many jets, J. High Energy Phys. 11 (2012) 081.

[12] J. Fan, M. Reece, and J. T. Ruderman, Stealth supersymmetry, J. High Energy Phys. 11 (2011) 012.

[13] G. F. Giudice and R. Rattazzi, Theories with gauge mediated supersymmetry breaking, Phys. Rep. 322, 419 (1999).

[14] S. P. Martin, Compressed supersymmetry and natural neutralino dark matter from top squark-mediated annihilation to top quarks, Phys. Rev. D 75, 115005 (2007).

[15] T. J. LeCompte and S. P. Martin, Large hadron collider reach for supersymmetric models with compressed mass spectra, Phys. Rev. D 84, 015004 (2011). 
[16] M. J. Strassler, Why unparticle models with mass gaps are examples of hidden valleys, arXiv:0801.0629.

[17] CMS Collaboration, Search for supersymmetry in protonproton collisions at $13 \mathrm{TeV}$ using identified top quarks, Phys. Rev. D 97, 012007 (2018).

[18] CMS Collaboration, Search for direct production of supersymmetric partners of the top quark in the all-jets final state in proton-proton collisions at $\sqrt{s}=13 \mathrm{TeV}$, J. High Energy Phys. 10 (2017) 005.

[19] CMS Collaboration, Search for top squark pair production in pp collisions at $\sqrt{s}=13 \mathrm{TeV}$ using single lepton events, J. High Energy Phys. 10 (2017) 019.

[20] CMS Collaboration, Search for top squarks and dark matter particles, in opposite-charge dilepton final states at $\sqrt{s}=13$ TeV, Phys. Rev. D 97, 032009 (2018).

[21] ATLAS Collaboration, Search for a scalar partner of the top quark in the jets plus missing transverse momentum final state at $\sqrt{s}=13 \mathrm{TeV}$ with the ATLAS detector, J. High Energy Phys. 12 (2017) 085.

[22] ATLAS Collaboration, Search for top-squark pair production in final states with one lepton, jets, and missing transverse momentum using $36 \mathrm{fb}^{-1}$ of $\sqrt{s}=13 \mathrm{TeV}$ pp collision data with the ATLAS detector, J. High Energy Phys. 06 (2018) 108.

[23] J. Fan, M. Reece, and J. T. Ruderman, A stealth supersymmetry sampler, J. High Energy Phys. 07 (2012) 196.

[24] J. Fan, R. Krall, D. Pinner, M. Reece, and J. T. Ruderman, Stealth supersymmetry simplified, J. High Energy Phys. 07 (2016) 016.

[25] ATLAS Collaboration, A search for pair-produced resonances in four-jet final states at $\sqrt{s}=13 \mathrm{TeV}$ with the ATLAS detector, Eur. Phys. J. C 78, 250 (2018).

[26] CMS Collaboration, Search for pair-produced resonances decaying to quark pairs in proton-proton collisions at $\sqrt{s}=13$ TeV, Phys. Rev. D 98, 112014 (2018).

[27] ATLAS Collaboration, Search for B-L $R$-parity-violating top squarks in $\sqrt{s}=13 \mathrm{TeV}$ pp collisions with the ATLAS experiment, Phys. Rev. D 97, 032003 (2018).

[28] CMS Collaboration, Search for pair production of thirdgeneration scalar leptoquarks and top squarks in protonproton collisions at $\sqrt{s}=8 \mathrm{TeV}$, Phys. Lett. B 739, 229 (2014).

[29] CMS Collaboration, Search for $R$-parity violating decays of a top squark in proton-proton collisions at $\sqrt{s}=8 \mathrm{TeV}$, Phys. Lett. B 760, 178 (2016).

[30] ATLAS Collaboration, Search for new phenomena in a lepton plus high jet multiplicity final state with the ATLAS experiment using $\sqrt{s}=13 \mathrm{TeV}$ proton-proton collision data, J. High Energy Phys. 09 (2017) 088.

[31] CMS Collaboration, Search for stealth supersymmetry in events with jets, either photons or leptons, and low missing transverse momentum in pp collisions at $8 \mathrm{TeV}$, Phys. Lett. B 743, 503 (2015).

[32] CMS Collaboration, Search for supersymmetry in events with photons and low missing transverse energy in pp collisions at $\sqrt{s}=7 \mathrm{TeV}$, Phys. Lett. B 719, 42 (2013).

[33] J. A. Evans and Y. Kats, LHC coverage of RPV MSSM with light stops, J. High Energy Phys. 04 (2013) 028.

[34] CMS Collaboration, The CMS experiment at the CERN LHC, J. Instrum. 3, S08004 (2008).
[35] CMS Collaboration, The CMS trigger system, J. Instrum. 12, P01020 (2017).

[36] CMS Collaboration, Particle-flow reconstruction and global event description with the CMS detector, J. Instrum. 12, P10003 (2017).

[37] M. Cacciari, G. P. Salam, and G. Soyez, The anti- $k_{\mathrm{T}}$ jet clustering algorithm, J. High Energy Phys. 04 (2008) 063.

[38] M. Cacciari, G. P. Salam, and G. Soyez, FastJet user manual, Eur. Phys. J. C 72, 1896 (2012).

[39] CMS Collaboration, Technical proposal for the phase-II upgrade of the compact Muon solenoid, CMS Technical Proposal CERN-LHCC-2015-010, CMS-TDR-15-02, CERN, 2015, http://cds.cern.ch/record/2020886.

[40] CMS Collaboration, Performance of electron reconstruction and selection with the CMS detector in proton-proton collisions at $\sqrt{s}=8 \mathrm{TeV}$, J. Instrum. 10, P06005 (2015).

[41] CMS Collaboration, Performance of the CMS muon detector and muon reconstruction with proton-proton collisions at $\sqrt{s}=13 \mathrm{TeV}$, J. Instrum. 13, P06015 (2018).

[42] K. Rehermann and B. Tweedie, Efficient identification of boosted semileptonic top quarks at the LHC, J. High Energy Phys. 03 (2011) 059.

[43] CMS Collaboration, Jet performance in pp collisions at 7 TeV, CMS Physics Analysis Summary CMS-PAS-JME10-003 (2010), https://cds.cern.ch/record/1279362.

[44] CMS Collaboration, Jet algorithms performance in $13 \mathrm{TeV}$ data, CMS Physics Analysis Summary CMS-PAS-JME-16003 (2017), http://cds.cern.ch/record/2256875.

[45] CMS Collaboration, Jet energy scale and resolution in the CMS experiment in pp collisions at $8 \mathrm{TeV}$, J. Instrum. 12, P02014 (2017).

[46] CMS Collaboration, Jet energy scale and resolution performance with $13 \mathrm{TeV}$ data collected by CMS in 2016-2018, CMS Detector Performance Note CMS-DP-2020-019, 2020, https://cdsweb.cern.ch/record/2715872.

[47] M. Cacciari and G. P. Salam, Pileup subtraction using jet areas, Phys. Lett. B 659, 119 (2008).

[48] CMS Collaboration, Identification of heavy-flavour jets with the CMS detector in pp collisions at $13 \mathrm{TeV}$, J. Instrum. 13, P05011 (2018).

[49] P. Nason, A new method for combining NLO QCD with shower Monte Carlo algorithms, J. High Energy Phys. 11 (2004) 040.

[50] S. Frixione, P. Nason, and C. Oleari, Matching NLO QCD computations with parton shower simulations: The POWHEG method, J. High Energy Phys. 11 (2007) 070.

[51] S. Alioli, P. Nason, C. Oleari, and E. Re, A general framework for implementing NLO calculations in shower Monte Carlo programs: The POWHEG box, J. High Energy Phys. 06 (2010) 043.

[52] S. Frixione, P. Nason, and G. Ridolfi, A positive-weight next-to-leading-order Monte Carlo for heavy flavour hadroproduction, J. High Energy Phys. 09 (2007) 126.

[53] R. Frederix, E. Re, and P. Torrielli, Single-top t-channel hadroproduction in the four-flavour scheme with POWHEG and aMC@NLO, J. High Energy Phys. 09 (2012) 130.

[54] J. Alwall, R. Frederix, S. Frixione, V. Hirschi, F. Maltoni, O. Mattelaer, H. S. Shao, T. Stelzer, P. Torrielli, and M. Zaro, The automated computation of tree-level and next-toleading order differential cross sections, and their matching 
to parton shower simulations, J. High Energy Phys. 07 (2014) 079.

[55] A. Kalogeropoulos and J. Alwall, The SysCalc code: A tool to derive theoretical systematic uncertainties, arXiv:1801 .08401 .

[56] T. Sjöstrand, S. Ask, J. R. Christiansen, R. Corke, N. Desai, P. Ilten, S. Mrenna, S. Prestel, C. O. Rasmussen, and P.Z. Skands, An introduction to PYTHIA 8.2, Comput. Phys. Commun. 191, 159 (2015).

[57] C. Borschensky, M. Krämer, A. Kulesza, M. Mangano, S. Padhi, T. Plehn, and X. Portell, Squark and gluino production cross sections in pp collisions at $\sqrt{s}=13,14,33$ and $100 \mathrm{TeV}$, Eur. Phys. J. C 74, 3174 (2014).

[58] W. Beenakker, C. Borschensky, M. Krämer, A. Kulesza, and E. Laenen, NNLL-fast: Predictions for coloured supersymmetric particle production at the LHC with threshold and Coulomb resummation, J. High Energy Phys. 12 (2016) 133.

[59] R. D. Ball et al. (NNPDF Collaboration), Parton distributions for the LHC Run II, J. High Energy Phys. 04 (2015) 040 .

[60] R. D. Ball et al. (NNPDF Collaboration), Parton distributions from high-precision collider data, Eur. Phys. J. C 77, 663 (2017).

[61] CMS Collaboration, Event generator tunes obtained from underlying event and multiparton scattering measurements, Eur. Phys. J. C 76, 155 (2016).

[62] CMS Collaboration, Investigations of the impact of the parton shower tuning in PYTHIA 8 in the modelling of $t \bar{t}$ at $\sqrt{s}=8$ and $13 \mathrm{TeV}$, CMS Physics Analysis Summary CMS-PAS-TOP-16-021, 2016, http://cds.cern.ch/record/ 2235192.

[63] CMS Collaboration, Extraction and validation of a new set of CMS PYTHIA 8 tunes from underlying-event measurements, Eur. Phys. J. C 80, 4 (2020).

[64] S. Agostinelli et al. (GEANT4 Collaboration), GEANT4-A simulation toolkit, Nucl. Instrum. Methods Phys. Res., Sect. A 506, 250 (2003).

[65] M. Czakon and A. Mitov, Top++: A program for the calculation of the top-pair cross-section at hadron colliders, Comput. Phys. Commun. 185, 2930 (2014).

[66] P. Kant, O. M. Kind, T. Kintscher, T. Lohse, T. Martini, S. Mölbitz, P. Rieck, and P. Uwer, HATHOR for single topquark production: Updated predictions and uncertainty estimates for single top-quark production in hadronic collisions, Comput. Phys. Commun. 191, 74 (2015).

[67] M. Aliev, H. Lacker, U. Langenfeld, S. Moch, P. Uwer, and M. Wiedermann, HATHOR: HAdronic Top and Heavy quarks crOss section calculatoR, Comput. Phys. Commun. 182, 1034 (2011).

[68] T. Gehrmann, M. Grazzini, S. Kallweit, P. Maierhöfer, A. von Manteuffel, S. Pozzorini, D. Rathlev, and L. Tancredi, $W^{+} \mathrm{W}^{-}$Production at Hadron Colliders in Next to Next to Leading Order QCD, Phys. Rev. Lett. 113, 212001 (2014).

[69] J. M. Campbell and R. K. Ellis, An update on vector boson pair production at hadron colliders, Phys. Rev. D 60, 113006 (1999).
[70] J. M. Campbell, R. K. Ellis, and C. Williams, Vector boson pair production at the LHC, J. High Energy Phys. 07 (2011) 018.

[71] Y. Li and F. Petriello, Combining QCD and electroweak corrections to dilepton production in FEWZ, Phys. Rev. D 86, 094034 (2012).

[72] Y. Ganin and V. Lempitsky, Unsupervised domain adaptation by backpropagation, arXiv:1409.7495.

[73] G. C. Fox and S. Wolfram, Observables for the Analysis of Event Shapes in $e^{+} e^{-}$Annihilation and Other Processes, Phys. Rev. Lett. 41, 1581 (1978).

[74] J. D. Bjorken and S. J. Brodsky, Statistical model for electronpositron annihilation into hadrons, Phys. Rev. D1, 1416(1970).

[75] E. Gerwick, T. Plehn, S. Schumann, and P. Schichtel, Scaling patterns for QCD jets, J. High Energy Phys. 10 (2012) 162.

[76] M. Cacciari, S. Frixione, M. L. Mangano, P. Nason, and G. Ridolfi, The $t \bar{t}$ cross-section at $1.8 \mathrm{TeV}$ and $1.96 \mathrm{TeV}$ : A study of the systematics due to parton densities and scale dependence, J. High Energy Phys. 04 (2004) 068.

[77] S. Catani, D. de Florian, M. Grazzini, and P. Nason, Soft gluon resummation for Higgs boson production at hadron colliders, J. High Energy Phys. 07 (2003) 028.

[78] CMS Collaboration, Measurement of the inelastic protonproton cross section at $\sqrt{s}=13 \mathrm{TeV}$, J. High Energy Phys. 07 (2018) 161.

[79] CMS Collaboration, CMS luminosity measurements for the 2016 data taking period, CMS Physics Analysis Summary, Technical Report No. CMS-PAS-LUM-17-001, 2017, https://cds.cern.ch/record/2257069.

[80] CMS Collaboration, CMS luminosity measurements for the 2017 data taking period at $\sqrt{s}=13 \mathrm{TeV}$, CMS Physics Analysis Summary, Technical Report No. CMS-PAS-LUM17-004, 2017, https://cds.cern.ch/record/2621960.

[81] CMS Collaboration, CMS luminosity measurements for the 2018 data taking period at $\sqrt{s}=13 \mathrm{TeV}$, CMS Physics Analysis Summary, Technical Report No. CMS-PAS-LUM18-002, 2018, https://cds.cern.ch/record/2676164.

[82] L. Demortier, $p$-values and nuisance parameters, in Statistical Issues for LHC Physics. Proceedings, Workshop, PHYSTATLHC, Geneva, Switzerland, 2007 ( CERN, Geneva, 2008), p. 23, http://dx.doi.org/10.5170/CERN-2008-001.

[83] ATLAS and CMS Collaborations, The LHC Higgs Combination Group, Procedure for the LHC Higgs boson search combination in Summer 2011, Technical Report No. CMSNOTE-2011-005, ATL-PHYS-PUB-2011-11, 2011, https:// cds.cern.ch/record/1379837.

[84] T. Junk, Confidence level computation for combining searches with small statistics, Nucl. Instrum. Methods Phys. Res., Sect. A 434, 435 (1999).

[85] A. L. Read, Presentation of search results: The $\mathrm{CL}_{\mathrm{s}}$ technique, J. Phys. G 28, 2693 (2002).

[86] G. Cowan, K. Cranmer, E. Gross, and O. Vitells, Asymptotic formulae for likelihood-based tests of new physics, Eur. Phys. J. C 71, 1554 (2011); Erratum, Eur. Phys. J. C 73, 2501 (2013). 
A. M. Sirunyan, ${ }^{1, a}$ A. Tumasyan, ${ }^{1}$ W. Adam, ${ }^{2}$ J. W. Andrejkovic, ${ }^{2}$ T. Bergauer, ${ }^{2}$ S. Chatterjee, ${ }^{2}$ M. Dragicevic, ${ }^{2}$ A. Escalante Del Valle, ${ }^{2}$ R. Frühwirth, ${ }^{2, b}$ M. Jeitler, ${ }^{2, b}$ N. Krammer, ${ }^{2}$ L. Lechner, ${ }^{2}$ D. Liko, ${ }^{2}$ I. Mikulec, ${ }^{2}$ F. M. Pitters, ${ }^{2}$ J. Schieck, ${ }^{2, b}$ R. Schöfbeck, ${ }^{2}$ M. Spanring, ${ }^{2}$ S. Templ, ${ }^{2}$ W. Waltenberger, ${ }^{2}$ C.-E. Wulz, ${ }^{2, b}$ V. Chekhovsky, ${ }^{3}$ A. Litomin, ${ }^{3}$ V. Makarenko, ${ }^{3}$ M. R. Darwish, ${ }^{4, \mathrm{c}}$ E. A. De Wolf, ${ }^{4}$ X. Janssen, ${ }^{4}$ T. Kello, ${ }^{4, \mathrm{~d}}$ A. Lelek, ${ }^{4}$ H. Rejeb Sfar, ${ }^{4}$ P. Van Mechelen, ${ }^{4}$ S. Van Putte, ${ }^{4}$ N. Van Remortel, ${ }^{4}$ F. Blekman, ${ }^{5}$ E. S. Bols, ${ }^{5}$ J. D’Hondt, ${ }^{5}$ J. De Clercq, ${ }^{5}$ M. Delcourt, ${ }^{5}$ S. Lowette, ${ }^{5}$ S. Moortgat, ${ }^{5}$ A. Morton, ${ }^{5}$ D. Müller, ${ }^{5}$ A. R. Sahasransu, ${ }^{5}$ S. Tavernier, ${ }^{5}$ W. Van Doninck, ${ }^{5}$ P. Van Mulders, ${ }^{5}$ D. Beghin, ${ }^{6}$ B. Bilin, ${ }^{6}$ B. Clerbaux, ${ }^{6}$ G. De Lentdecker, ${ }^{6}$ L. Favart, ${ }^{6}$ A. Grebenyuk, ${ }^{6}$ A. K. Kalsi, ${ }^{6}$ K. Lee, ${ }^{6}$ M. Mahdavikhorrami, ${ }^{6}$

I. Makarenko, ${ }^{6}$ L. Moureaux, ${ }^{6}$ L. Pétré, ${ }^{6}$ A. Popov, ${ }^{6}$ N. Postiau, ${ }^{6}$ E. Starling, ${ }^{6}$ L. Thomas, ${ }^{6}$ M. Vanden Bemden, ${ }^{6}$ C. Vander Velde, ${ }^{6}$ P. Vanlaer, ${ }^{6}$ D. Vannerom, ${ }^{6}$ L. Wezenbeek, ${ }^{6}$ T. Cornelis, ${ }^{7}$ D. Dobur, ${ }^{7}$ M. Gruchala, ${ }^{7}$ G. Mestdach, ${ }^{7}$ M. Niedziela, ${ }^{7}$ C. Roskas, ${ }^{7}$ K. Skovpen, ${ }^{7}$ M. Tytgat, ${ }^{7}$ W. Verbeke, ${ }^{7}$ B. Vermassen, ${ }^{7}$ M. Vit, ${ }^{7}$ A. Bethani,${ }^{8}$ G. Bruno, ${ }^{8}$ F. Bury, ${ }^{8}$ C. Caputo, ${ }^{8}$ P. David,${ }^{8}$ C. Delaere, ${ }^{8}$ I. S. Donertas, ${ }^{8}$ A. Giammanco, ${ }^{8}$ V. Lemaitre, ${ }^{8}$ K. Mondal, ${ }^{8}$ J. Prisciandaro, ${ }^{8}$ A. Taliercio, ${ }^{8}$ M. Teklishyn, ${ }^{8}$ P. Vischia, ${ }^{8}$ S. Wertz,${ }^{8}$ S. Wuyckens, ${ }^{8}$ G. A. Alves, ${ }^{9}$ C. Hensel, ${ }^{9}$ A. Moraes,${ }^{9}$ W. L. Aldá Júnior, ${ }^{10}$ M. Barroso Ferreira Filho, ${ }^{10}$ H. Brandao Malbouisson, ${ }^{10}$ W. Carvalho, ${ }^{10}$ J. Chinellato, ${ }^{10, e}$ E. M. Da Costa, ${ }^{10}$ G. G. Da Silveira, ${ }^{10, \mathrm{f}}$ D. De Jesus Damiao, ${ }^{10}$ S. Fonseca De Souza, ${ }^{10}$ D. Matos Figueiredo, ${ }^{10}$ C. Mora Herrera, ${ }^{10}$ K. Mota Amarilo, ${ }^{10}$ L. Mundim, ${ }^{10}$ H. Nogima, ${ }^{10}$ P. Rebello Teles, ${ }^{10}$ L. J. Sanchez Rosas, ${ }^{10}$ A. Santoro, ${ }^{10}$ S. M. Silva Do Amaral, ${ }^{10}$ A. Sznajder, ${ }^{10}$ M. Thiel,${ }^{10}$ F. Torres Da Silva De Araujo, ${ }^{10}$ A. Vilela Pereira, ${ }^{10}$ C. A. Bernardes, ${ }^{11 a}$

L. Calligaris, ${ }^{11 \mathrm{a}}$ T. R. Fernandez Perez Tomei, ${ }^{11 \mathrm{a}}$ E. M. Gregores, ${ }^{11 \mathrm{a}, 1 \mathrm{~b}}$ D. S. Lemos, ${ }^{11 \mathrm{a}}$ P. G. Mercadante, ${ }^{11 \mathrm{a}, 1 \mathrm{~b}}$

S. F. Novaes, ${ }^{11 a}$ Sandra S. Padula, ${ }^{11 a}$ A. Aleksandrov, ${ }^{12}$ G. Antchev, ${ }^{12}$ I. Atanasov,${ }^{12}$ R. Hadjiiska, ${ }^{12}$ P. Iaydjiev, ${ }^{12}$ M. Misheva, ${ }^{12}$ M. Rodozov, ${ }^{12}$ M. Shopova, ${ }^{12}$ G. Sultanov, ${ }^{12}$ A. Dimitrov, ${ }^{13}$ T. Ivanov, ${ }^{13}$ L. Litov, ${ }^{13}$ B. Pavlov,${ }^{13}$ P. Petkov, ${ }^{13}$ A. Petrov, ${ }^{13}$ T. Cheng, ${ }^{14}$ W. Fang, ${ }^{14, d}$ Q. Guo, ${ }^{14}$ T. Javaid, ${ }^{14, g}$ M. Mittal, ${ }^{14}$ H. Wang,,${ }^{14}$ L. Yuan, ${ }^{14}$ M. Ahmad,${ }^{15}$ G. Bauer, ${ }^{15}$ C. Dozen, ${ }^{15, \mathrm{~h}}$ Z. Hu, ${ }^{15}$ J. Martins, ${ }^{15, \mathrm{i}}$ Y. Wang, ${ }^{15}$ K. Yi, ${ }^{15, \mathrm{j}, \mathrm{k}}$ E. Chapon, ${ }^{16}$ G. M. Chen, ${ }^{16, \mathrm{~g}}$ H. S. Chen, ${ }^{16, \mathrm{~g}}$ M. Chen, ${ }^{16}$ A. Kapoor, ${ }^{16}$ D. Leggat, ${ }^{16}$ H. Liao, ${ }^{16}$ Z.-A. LIU, ${ }^{16,1}$ R. Sharma, ${ }^{16}$ A. Spiezia, ${ }^{16}$ J. Tao, ${ }^{16}$ J. Thomas-Wilsker, ${ }^{16}$ J. Wang, ${ }^{16}$ H. Zhang, ${ }^{16}$ S. Zhang, ${ }^{16,9}$ J. Zhao, ${ }^{16}$ A. Agapitos, ${ }^{17}$ Y. Ban, ${ }^{17}$ C. Chen,${ }^{17}$ Q. Huang, ${ }^{17}$ A. Levin, ${ }^{17}$ Q. Li,${ }^{17}$ M. Lu, ${ }^{17}$ X. Lyu,${ }^{17}$ Y. Mao, ${ }^{17}$ S. J. Qian, ${ }^{17}$ D. Wang, ${ }^{17}$ Q. Wang,${ }^{17}$ J. Xiao, ${ }^{17}$ Z. You, ${ }^{18}$ X. Gao,${ }^{19,}$ H. Okawa, ${ }^{19}$ M. Xiao, ${ }^{20}$ C. Avila, ${ }^{21}$ A. Cabrera, ${ }^{21}$ C. Florez, ${ }^{21}$ J. Fraga, ${ }^{21}$ A. Sarkar, ${ }^{21}$ M. A. Segura Delgado, ${ }^{21}$ J. Jaramillo, ${ }^{22}$ J. Mejia Guisao, ${ }^{22}$ F. Ramirez, ${ }^{22}$ J. D. Ruiz Alvarez, ${ }^{22}$ C. A. Salazar González, ${ }^{22}$ N. Vanegas Arbelaez, ${ }^{22}$ D. Giljanovic, ${ }^{23}$ N. Godinovic, ${ }^{23}$ D. Lelas, ${ }^{23}$ I. Puljak, ${ }^{23}$ Z. Antunovic, ${ }^{24}$ M. Kovac, ${ }^{24}$ T. Sculac, ${ }^{24}$ V. Brigljevic, ${ }^{25}$ D. Ferencek, ${ }^{25}$ D. Majumder, ${ }^{25}$ M. Roguljic, ${ }^{25}$ A. Starodumov, ${ }^{25, \mathrm{~m}}$ T. Susa, ${ }^{25}$ A. Attikis, ${ }^{26}$ E. Erodotou, ${ }^{26}$ A. Ioannou, ${ }^{26}$ G. Kole, ${ }^{26}$ M. Kolosova, ${ }^{26}$ S. Konstantinou, ${ }^{26}$ J. Mousa, ${ }^{26}$ C. Nicolaou, ${ }^{26}$ F. Ptochos,${ }^{26}$ P. A. Razis, ${ }^{26}$ H. Rykaczewski, ${ }^{26}$ H. Saka, ${ }^{26}$ M. Finger, ${ }^{27, n}$ M. Finger Jr., ${ }^{27, n}$ A. Kveton, ${ }^{27}$ E. Ayala, ${ }^{28}$ E. Carrera Jarrin, ${ }^{29}$ S. Abu Zeid, ${ }^{30, o}$ S. Khalil,,${ }^{30, p}$ E. Salama, ${ }^{30, q, o}$ A. Lotfy, ${ }^{31}$ Y. Mohammed, ${ }^{31}$ S. Bhowmik, ${ }^{32}$ A. Carvalho Antunes De Oliveira, ${ }^{32}$ R. K. Dewanjee, ${ }^{32}$ K. Ehataht, ${ }^{32}$ M. Kadastik, ${ }^{32}$ J. Pata, ${ }^{32}$ M. Raidal, ${ }^{32}$ C. Veelken, ${ }^{32}$ P. Eerola, ${ }^{33}$ L. Forthomme,${ }^{33}$ H. Kirschenmann, ${ }^{33}$ K. Osterberg, ${ }^{33}$ M. Voutilainen, ${ }^{33}$ E. Brücken,${ }^{34}$ F. Garcia, ${ }^{34}$ J. Havukainen,${ }^{34}$ V. Karimäki, ${ }^{34}$ M. S. Kim, ${ }^{34}$ R. Kinnunen, ${ }^{34}$ T. Lampén, ${ }^{34}$ K. Lassila-Perini, ${ }^{34}$ S. Lehti, ${ }^{34}$ T. Lindén, ${ }^{34}$ H. Siikonen, ${ }^{34}$ E. Tuominen, ${ }^{34}$ J. Tuominiemi, ${ }^{34}$ P. Luukka, ${ }^{35}$ H. Petrow,${ }^{35}$ T. Tuuva, ${ }^{35}$ C. Amendola, ${ }^{36}$ M. Besancon, ${ }^{36}$ F. Couderc, ${ }^{36}$ M. Dejardin, ${ }^{36}$ D. Denegri, ${ }^{36}$ J. L. Faure, ${ }^{36}$ F. Ferri, ${ }^{36}$ S. Ganjour, ${ }^{36}$ A. Givernaud, ${ }^{36}$ P. Gras, ${ }^{36}$ G. Hamel de Monchenault, ${ }^{36}$ P. Jarry, ${ }^{36}$ B. Lenzi, ${ }^{36}$ E. Locci, ${ }^{36}$ J. Malcles, ${ }^{36}$ J. Rander, ${ }^{36}$ A. Rosowsky, ${ }^{36}$ M. Ö. Sahin, ${ }^{36}$ A. Savoy-Navarro, ${ }^{36, \mathrm{r}}$ M. Titov, ${ }^{36}$ G. B. Yu, ${ }^{36}$ S. Ahuja, ${ }^{37}$ F. Beaudette, ${ }^{37}$ M. Bonanomi, ${ }^{37}$ A. Buchot Perraguin, ${ }^{37}$ P. Busson, ${ }^{37}$ C. Charlot, ${ }^{37}$ O. Davignon, ${ }^{37}$ B. Diab,${ }^{37}$ G. Falmagne, ${ }^{37}$ S. Ghosh, ${ }^{37}$ R. Granier de Cassagnac, ${ }^{37}$ A. Hakimi, ${ }^{37}$ I. Kucher, ${ }^{37}$ A. Lobanov, ${ }^{37}$ M. Nguyen, ${ }^{37}$ C. Ochando, ${ }^{37}$ P. Paganini, ${ }^{37}$ J. Rembser, ${ }^{37}$ R. Salerno, ${ }^{37}$ J. B. Sauvan, ${ }^{37}$ Y. Sirois,${ }^{37}$ A. Zabi, ${ }^{37}$ A. Zghiche, ${ }^{37}$ J.-L. Agram, ${ }^{38, s}$ J. Andrea, ${ }^{38}$ D. Apparu, ${ }^{38}$ D. Bloch, ${ }^{38}$ G. Bourgatte, ${ }^{38}$ J.-M. Brom, ${ }^{38}$ E. C. Chabert, ${ }^{38}$ C. Collard, ${ }^{38}$ D. Darej, ${ }^{38}$ J.-C. Fontaine, ${ }^{38, \mathrm{~s}}$ U. Goerlach, ${ }^{38}$ C. Grimault, ${ }^{38}$ A.-C. Le Bihan, ${ }^{38}$ P. Van Hove, ${ }^{38}$ E. Asilar, ${ }^{39}$ S. Beauceron, ${ }^{39}$ C. Bernet, ${ }^{39}$ G. Boudoul, ${ }^{39}$ C. Camen, ${ }^{39}$ A. Carle,${ }^{39}$ N. Chanon, ${ }^{39}$ D. Contardo, ${ }^{39}$ P. Depasse,${ }^{39}$

H. El Mamouni, ${ }^{39}$ J. Fay, ${ }^{39}$ S. Gascon, ${ }^{39}$ M. Gouzevitch, ${ }^{39}$ B. Ille, ${ }^{39}$ Sa. Jain, ${ }^{39}$ I. B. Laktineh, ${ }^{39}$ H. Lattaud, ${ }^{39}$

A. Lesauvage, ${ }^{39}$ M. Lethuillier, ${ }^{39}$ L. Mirabito, ${ }^{39}$ K. Shchablo, ${ }^{39}$ L. Torterotot,${ }^{39}$ G. Touquet, ${ }^{39}$ M. Vander Donckt, ${ }^{39}$ S. Viret, ${ }^{39}$ G. Adamov, ${ }^{40}$ Z. Tsamalaidze ${ }^{40, n}$ L. Feld, ${ }^{41}$ K. Klein, ${ }^{41}$ M. Lipinski, ${ }^{41}$ D. Meuser, ${ }^{41}$ A. Pauls, ${ }^{41}$ M. P. Rauch, ${ }^{41}$ J. Schulz ${ }^{41}$ M. Teroerde, ${ }^{41}$ D. Eliseev, ${ }^{42}$ M. Erdmann, ${ }^{42}$ P. Fackeldey ${ }^{42}$ B. Fischer, ${ }^{42}$ S. Ghosh,${ }^{42}$ T. Hebbeker, ${ }^{42}$

K. Hoepfner, ${ }^{42}$ H. Keller, ${ }^{42}$ L. Mastrolorenzo, ${ }^{42}$ M. Merschmeyer, ${ }^{42}$ A. Meyer, ${ }^{42}$ G. Mocellin, ${ }^{42}$ S. Mondal, ${ }^{42}$

S. Mukherjee, ${ }^{42}$ D. Noll, ${ }^{42}$ A. Novak, ${ }^{42}$ T. Pook, ${ }^{42}$ A. Pozdnyakov, ${ }^{42}$ Y. Rath,${ }^{42}$ H. Reithler, ${ }^{42}$ J. Roemer, ${ }^{42}$ A. Schmidt, ${ }^{42}$ S. C. Schuler, ${ }^{42}$ A. Sharma, ${ }^{42}$ S. Wiedenbeck, ${ }^{42}$ S. Zaleski, ${ }^{42}$ C. Dziwok,${ }^{43}$ G. Flügge, ${ }^{43}$ W. Haj Ahmad, ${ }^{43, t}$ 
O. Hlushchenko, ${ }^{43}$ T. Kress, ${ }^{43}$ A. Nowack, ${ }^{43}$ C. Pistone, ${ }^{43}$ O. Pooth, ${ }^{43}$ D. Roy, ${ }^{43}$ H. Sert, ${ }^{43}$ A. Stahl, ${ }^{43, u}$ T. Ziemons, ${ }^{43}$ H. Aarup Petersen, ${ }^{44}$ M. Aldaya Martin, ${ }^{44}$ P. Asmuss, ${ }^{44}$ I. Babounikau, ${ }^{44}$ S. Baxter, ${ }^{44}$ O. Behnke, ${ }^{44}$ A. Bermúdez Martínez, ${ }^{44}$ A. A. Bin Anuar, ${ }^{44}$ K. Borras, ${ }^{44, v}$ V. Botta, ${ }^{44}$ D. Brunner, ${ }^{44}$ A. Campbell, ${ }^{44}$ A. Cardini, ${ }^{44}$ P. Connor, ${ }^{44}$

S. Consuegra Rodríguez, ${ }^{44}$ V. Danilov, ${ }^{44}$ M. M. Defranchis, ${ }^{44}$ L. Didukh, ${ }^{44}$ D. Domínguez Damiani, ${ }^{44}$ G. Eckerlin, ${ }^{44}$ D. Eckstein, ${ }^{44}$ L. I. Estevez Banos, ${ }^{44}$ E. Gallo, ${ }^{4, w}$ A. Geiser, ${ }^{44}$ A. Giraldi, ${ }^{44}$ A. Grohsjean, ${ }^{44}$ M. Guthoff, ${ }^{44}$ A. Harb, ${ }^{44}$ A. Jafari, ${ }^{44, x}$ N. Z. Jomhari, ${ }^{44}$ H. Jung, ${ }^{44}$ A. Kasem, ${ }^{44, v}$ M. Kasemann, ${ }^{44}$ H. Kaveh, ${ }^{44}$ C. Kleinwort, ${ }^{44}$ J. Knolle, $^{44}$ D. Krücker, ${ }^{44}$ W. Lange, ${ }^{44}$ T. Lenz, ${ }^{44}$ J. Lidrych, ${ }^{44}$ K. Lipka, ${ }^{44}$ W. Lohmann, ${ }^{44, y}$ T. Madlener, ${ }^{44}$ R. Mankel, ${ }^{44}$ I.-A. Melzer-Pellmann, ${ }^{44}$ J. Metwally, ${ }^{44}$ A. B. Meyer, ${ }^{44}$ M. Meyer, ${ }^{44}$ J. Mnich, ${ }^{44}$ A. Mussgiller, ${ }^{44}$ V. Myronenko, ${ }^{44}$ Y. Otarid, ${ }^{44}$ D. Pérez Adán, ${ }^{44}$ S. K. Pflitsch, ${ }^{44}$ D. Pitzl, ${ }^{44}$ A. Raspereza, ${ }^{44}$ A. Saggio, ${ }^{44}$ A. Saibel, ${ }^{44}$ M. Savitskyi, ${ }^{44}$ V. Scheurer, ${ }^{44}$ C. Schwanenberger, ${ }^{44}$ A. Singh, ${ }^{44}$ R. E. Sosa Ricardo, ${ }^{44}$ N. Tonon, ${ }^{44}$ O. Turkot, ${ }^{44}$ A. Vagnerini, ${ }^{44}$ M. Van De Klundert, ${ }^{44}$ R. Walsh, ${ }^{44}$ D. Walter, ${ }^{44}$ Y. Wen, ${ }^{44}$ K. Wichmann, ${ }^{44}$ C. Wissing, ${ }^{44}$ S. Wuchterl, $^{44}$ O. Zenaiev, ${ }^{44}$ R. Zlebcik, ${ }^{44}$ R. Aggleton, ${ }^{45}$ S. Bein, ${ }^{45}$ L. Benato, ${ }^{45}$ A. Benecke, ${ }^{45}$ K. De Leo, ${ }^{45}$ T. Dreyer, ${ }^{45}$ M. Eich, ${ }^{45}$ F. Feindt, ${ }^{45}$ A. Fröhlich, ${ }^{45}$ C. Garbers, ${ }^{45}$ E. Garutti, ${ }^{45}$ P. Gunnellini, ${ }^{45}$ J. Haller, ${ }^{45}$ A. Hinzmann, ${ }^{45}$ A. Karavdina, ${ }^{45}$ G. Kasieczka, ${ }^{45}$ R. Klanner, ${ }^{45}$ R. Kogler, ${ }^{45}$ V. Kutzner, ${ }^{45}$ J. Lange, ${ }^{45}$ T. Lange, ${ }^{45}$ A. Malara, ${ }^{45}$ A. Nigamova, ${ }^{45}$ K. J. Pena Rodriguez, ${ }^{45}$ O. Rieger, ${ }^{45}$ P. Schleper, ${ }^{45}$ M. Schröder, ${ }^{45}$ J. Schwandt, ${ }^{45}$ D. Schwarz, ${ }^{45}$ J. Sonneveld, ${ }^{45}$ H. Stadie, ${ }^{45}$ G. Steinbrück, ${ }^{45}$ A. Tews, ${ }^{45}$ B. Vormwald, ${ }^{45}$ I. Zoi, ${ }^{45}$ J. Bechtel, ${ }^{46}$ T. Berger, ${ }^{46}$ E. Butz, ${ }^{46}$ R. Caspart, ${ }^{46}$ T. Chwalek, $^{46}$ W. De Boer, ${ }^{46}$ A. Dierlamm, ${ }^{46}$ A. Droll, ${ }^{46}$ K. El Morabit, ${ }^{46}$ N. Faltermann, ${ }^{46}$ K. Flöh, ${ }^{46}$ M. Giffels, ${ }^{46}$ J. o. Gosewisch, ${ }^{46}$ A. Gottmann, ${ }^{46}$ F. Hartmann, ${ }^{46, u}$ C. Heidecker, ${ }^{46}$ U. Husemann, ${ }^{46}$ I. Katkov, ${ }^{46, z}$ P. Keicher, ${ }^{46}$ R. Koppenhöfer, ${ }^{46}$ S. Maier, ${ }^{46}$ M. Metzler, ${ }^{46}$ S. Mitra, ${ }^{46}$ Th. Müller, ${ }^{46}$ M. Musich, ${ }^{46}$ M. Neukum, ${ }^{46}$ G. Quast, ${ }^{46}$ K. Rabbertz, ${ }^{46}$ J. Rauser, $^{46}$ D. Savoiu, ${ }^{46}$ D. Schäfer, ${ }^{46}$ M. Schnepf, ${ }^{46}$ D. Seith, ${ }^{46}$ I. Shvetsov, ${ }^{46}$ H. J. Simonis, ${ }^{46}$ R. Ulrich, ${ }^{46}$ J. Van Der Linden, ${ }^{46}$ R. F. Von Cube, ${ }^{46}$ M. Wassmer, ${ }^{46}$ M. Weber, ${ }^{46}$ S. Wieland, ${ }^{46}$ R. Wolf, ${ }^{46}$ S. Wozniewski, ${ }^{46}$ S. Wunsch, ${ }^{46}$ G. Anagnostou, ${ }^{47}$ P. Asenov, ${ }^{47}$ G. Daskalakis, ${ }^{47}$

T. Geralis, ${ }^{47}$ A. Kyriakis, ${ }^{47}$ D. Loukas, ${ }^{47}$ A. Stakia, ${ }^{47}$ M. Diamantopoulou, ${ }^{48}$ D. Karasavvas, ${ }^{48}$ G. Karathanasis, ${ }^{48}$ P. Kontaxakis, ${ }^{48}$ C. K. Koraka, ${ }^{48}$ A. Manousakis-Katsikakis, ${ }^{48}$ A. Panagiotou, ${ }^{48}$ I. Papavergou, $^{48}$ N. Saoulidou, ${ }^{48}$

K. Theofilatos, ${ }^{48}$ E. Tziaferi, ${ }^{48}$ K. Vellidis, ${ }^{48}$ E. Vourliotis, ${ }^{48}$ G. Bakas, ${ }^{49}$ K. Kousouris, ${ }^{49}$ I. Papakrivopoulos, ${ }^{49}$ G. Tsipolitis, ${ }^{49}$ A. Zacharopoulou, ${ }^{49}$ I. Evangelou, ${ }^{50}$ C. Foudas, ${ }^{50}$ P. Gianneios, ${ }^{50}$ P. Katsoulis, ${ }^{50}$ P. Kokkas, ${ }^{50}$ N. Manthos, ${ }^{50}$ I. Papadopoulos, ${ }^{50}$ J. Strologas, ${ }^{50}$ M. Csanad, ${ }^{51}$ M. M. A. Gadallah, ${ }^{51, \text { aa }}$ S. Lökös, ${ }^{51, b b}$ P. Major, ${ }^{51}$ K. Mandal, ${ }^{51}$ A. Mehta, ${ }^{51}$ G. Pasztor, ${ }^{51}$ A. J. Rádl, ${ }^{51}$ O. Surányi, ${ }^{51}$ G. I. Veres, ${ }^{51}$ M. Bartók, ${ }^{52, c c}$ G. Bencze, ${ }^{52}$ C. Hajdu, ${ }^{52}$ D. Horvath, ${ }^{52, d d}$ F. Sikler, ${ }^{52}$ V. Veszpremi, ${ }^{52}$ G. Vesztergombi, ${ }^{52, \text { a,e }}$ S. Czellar, ${ }^{53}$ J. Karancsi, ${ }^{53, c c}$ J. Molnar, ${ }^{53}$ Z. Szillasi, ${ }^{53}$ D. Teyssier, ${ }^{53}$ P. Raics, ${ }^{54}$ Z. L. Trocsanyi, ${ }^{54, e e}$ B. Ujvari, ${ }^{54}$ T. Csorgo, ${ }^{55, f f}$ F. Nemes, ${ }^{55, f f}$ T. Novak, ${ }^{55}$ S. Choudhury, ${ }^{56}$ J. R. Komaragiri, ${ }^{56}$ D. Kumar, ${ }^{56}$ L. Panwar, ${ }^{56}$ P. C. Tiwari, ${ }^{56}$ S. Bahinipati, ${ }^{57, g g}$ D. Dash, ${ }^{57}$ C. Kar, ${ }^{57}$ P. Mal, ${ }^{57}$ T. Mishra, ${ }^{57}$

V. K. Muraleedharan Nair Bindhu, ${ }^{57, \text { hh }}$ A. Nayak, ${ }^{57, \text { hh }}$ P. Saha, ${ }^{57}$ N. Sur, ${ }^{57}$ S. K. Swain, ${ }^{57}$ S. Bansal, ${ }^{58}$ S. B. Beri, ${ }^{58}$ V. Bhatnagar, ${ }^{58}$ G. Chaudhary, ${ }^{58}$ S. Chauhan, ${ }^{58}$ N. Dhingra, ${ }^{58, i i}$ R. Gupta, ${ }^{58}$ A. Kaur, ${ }^{58}$ S. Kaur, ${ }^{58}$ P. Kumari, ${ }^{58}$ M. Meena, ${ }^{58}$ K. Sandeep, ${ }^{58}$ J. B. Singh, ${ }^{58}$ A. K. Virdi, ${ }^{58}$ A. Ahmed, ${ }^{59}$ A. Bhardwaj, ${ }^{59}$ B. C. Choudhary, ${ }^{59}$ R. B. Garg, ${ }^{59}$ M. Gola, ${ }^{59}$

S. Keshri, ${ }^{59}$ A. Kumar, ${ }^{59}$ M. Naimuddin, ${ }^{59}$ P. Priyanka, ${ }^{59}$ K. Ranjan, ${ }^{59}$ A. Shah, ${ }^{59}$ M. Bharti, ${ }^{60, j j}$ R. Bhattacharya, ${ }^{60}$ S. Bhattacharya, ${ }^{60}$ D. Bhowmik, ${ }^{60}$ S. Dutta, ${ }^{60}$ B. Gomber, ${ }^{60, k k}$ M. Maity, ${ }^{60,11}$ S. Nandan, ${ }^{60}$ P. Palit, ${ }^{60}$ P. K. Rout, ${ }^{60}$ G. Saha, ${ }^{60}$ B. Sahu, ${ }^{60}$ S. Sarkar, ${ }^{60}$ M. Sharan, ${ }^{60}$ B. Singh, ${ }^{60, j j}$ S. Thakur, ${ }^{60, j j}$ P. K. Behera, ${ }^{61}$ S. C. Behera, ${ }^{61}$ P. Kalbhor, ${ }^{61}$

A. Muhammad, ${ }^{61}$ R. Pradhan, ${ }^{61}$ P. R. Pujahari, ${ }^{61}$ A. Sharma, ${ }^{61}$ A. K. Sikdar, ${ }^{61}$ D. Dutta, ${ }^{62}$ V. Jha, ${ }^{62}$ V. Kumar, ${ }^{62}$ D. K. Mishra, ${ }^{62}$ K. Naskar, ${ }^{62, m m}$ P. K. Netrakanti, ${ }^{62}$ L. M. Pant, ${ }^{62}$ P. Shukla, ${ }^{62}$ T. Aziz, ${ }^{63}$ S. Dugad, ${ }^{63}$ G. B. Mohanty, ${ }^{63}$ U. Sarkar, ${ }^{63}$ S. Banerjee, ${ }^{64}$ S. Bhattacharya, ${ }^{64}$ R. Chudasama, ${ }^{64}$ M. Guchait, ${ }^{64}$ S. Karmakar, ${ }^{64}$ S. Kumar, ${ }^{64}$ G. Majumder, ${ }^{64}$ K. Mazumdar, ${ }^{64}$ S. Mukherjee, ${ }^{64}$ D. Roy, ${ }^{64}$ S. Dube, ${ }^{65}$ B. Kansal, ${ }^{65}$ S. Pandey, ${ }^{65}$ A. Rane, ${ }^{65}$ A. Rastogi, ${ }^{65}$ S. Sharma, ${ }^{65}$ H. Bakhshiansohi, ${ }^{66, n n}$ M. Zeinali, ${ }^{66,00}$ S. Chenarani, ${ }^{67, p p}$ S. M. Etesami, ${ }^{67}$ M. Khakzad, ${ }^{67}$ M. Mohammadi Najafabadi, ${ }^{67}$ M. Felcini, ${ }^{68}$ M. Grunewald, ${ }^{68}$ M. Abbrescia, ${ }^{69 a, 69 b}$ R. Aly, ${ }^{69 a, 69 b, q q}$ C. Aruta, ${ }^{69 a, 69 b}$ A. Colaleo, ${ }^{69 a}$ D. Creanza, ${ }^{69 a, 69 c}$ N. De Filippis, ${ }^{69 a, 69 c}$ M. De Palma, ${ }^{69 a, 69 b}$ A. Di Florio, ${ }^{69 a, 69 b}$ A. Di Pilato, ${ }^{69 a, 69 b}$ W. Elmetenawee, ${ }^{69 a, 69 b}$ L. Fiore, ${ }^{69 a}$ A. Gelmi, ${ }^{69 a, 69 b}$ M. Gul, ${ }^{69 a}$ G. Iaselli, ${ }^{69 a, 69 c}$ M. Ince, ${ }^{69 a, 69 b}$ S. Lezki, ${ }^{69 a, 69 b}$ G. Maggi, ${ }^{69 a, 69 c}$ M. Maggi, ${ }^{69 a}$ I. Margjeka, ${ }^{69 a, 69 b}$ V. Mastrapasqua, ${ }^{69 a, 69 b}$ J. A. Merlin, ${ }^{69 a}$ S. My, ${ }^{69 a, 69 b}$ S. Nuzzo, ${ }^{69 a, 69 b}$ A. Pompili, ${ }^{69 a, 69 b}$ G. Pugliese, ${ }^{69 a, 69 c}$ A. Ranieri, ${ }^{69 a}$ G. Selvaggi, ${ }^{69 a, 69 b}$ L. Silvestris, ${ }^{69 a}$ F. M. Simone, ${ }^{69 a, 69 b}$ R. Venditti, ${ }^{69 a}$ P. Verwilligen, ${ }^{69 a}$ G. Abbiendi, ${ }^{70 a}$ C. Battilana, ${ }^{70 a, 70 b}$ D. Bonacorsi, ${ }^{70 a, 70 b}$ L. Borgonovi, ${ }^{70 a}$ S. Braibant-Giacomelli, ${ }^{70 a, 70 b}$ L. Brigliadori, ${ }^{70 a}$ R. Campanini, ${ }^{70 a, 70 b}$ P. Capiluppi, ${ }^{70 a, 70 b}$ A. Castro, ${ }^{70 a, 70 b}$ F. R. Cavallo, ${ }^{70 a}$ C. Ciocca, ${ }^{70 a}$ M. Cuffiani, ${ }^{70 a, 70 b}$ G. M. Dallavalle, ${ }^{70 a}$ T. Diotalevi, ${ }^{70 a, 70 b}$ F. Fabbri, ${ }^{70 a}$ A. Fanfani, ${ }^{70 a, 70 b}$ E. Fontanesi, ${ }^{70 a, 70 b}$ P. Giacomelli, ${ }^{70 a}$ L. Giommi, ${ }^{70 a, 70 b}$ C. Grandi, ${ }^{70 a}$ L. Guiducci, ${ }^{70 a, 70 b}$ F. Iemmi, ${ }^{70 a, 70 b}$ 
S. Lo Meo, ${ }^{70 a, r r}$ S. Marcellini, ${ }^{70 a}$ G. Masetti, ${ }^{70 a}$ F. L. Navarria, ${ }^{70 a, 70 b}$ A. Perrotta ${ }^{70 a}$ F. Primavera, ${ }^{70 a, 70 b}$ A. M. Rossi ${ }^{70 a, 70 b}$ T. Rovelli, ${ }^{70 a, 70 b}$ G. P. Siroli, ${ }^{70 a, 70 b}$ N. Tosi, ${ }^{70 a}$ S. Albergo, ${ }^{71 a, 71 b, s s}$ S. Costa, ${ }^{71 a, 71 b, s s}$ A. Di Mattia, ${ }^{71 a}$ R. Potenza, ${ }^{71,71 b}$ A. Tricomi, ${ }^{71 a, 71 b, s s}$ C. Tuve, ${ }^{71 a, 71 b}$ G. Barbagli, ${ }^{72 a}$ A. Cassese, ${ }^{72 a}$ R. Ceccarelli, ${ }^{72 a, 72 b}$ V. Ciulli, ${ }^{72 a, 72 b}$ C. Civinini, ${ }^{72 a}$ R. D’Alessandro, ${ }^{72 a, 72 b}$ F. Fiori, ${ }^{72 a, 72 b}$ E. Focardi, ${ }^{72 a, 72 b}$ G. Latino, ${ }^{72 a, 72 b}$ P. Lenzi, ${ }^{72 a, 72 b}$ M. Lizzo, ${ }^{72 a, 72 b}$ M. Meschini, ${ }^{72 a}$ S. Paoletti, ${ }^{72 a}$ R. Seidita, ${ }^{72 a, 72 b}$ G. Sguazzoni,${ }^{72 a}$ L. Viliani, ${ }^{72 a}$ L. Benussi, ${ }^{73}$ S. Bianco, ${ }^{73}$ D. Piccolo, ${ }^{73}$ M. Bozzo, ${ }^{74 a, 74 b}$ F. Ferro, ${ }^{74 a}$ R. Mulargia, ${ }^{74 a, 74 b}$ E. Robutti ${ }^{74 a}$ S. Tosi, ${ }^{74 a, 74 b}$ A. Benaglia, ${ }^{75 a}$ F. Brivio, ${ }^{75 a, 75 b}$ F. Cetorelli, ${ }^{75 a, 75 b}$ V. Ciriolo ${ }^{75 a, 75 b, u}$ F. De Guio, ${ }^{75 a, 75 b}$ M. E. Dinardo, ${ }^{75 a, 75 b}$ P. Dini, ${ }^{75 a}$ S. Gennai, ${ }^{75 a}$ A. Ghezzi, ${ }^{75 a, 75 b}$ P. Govoni, ${ }^{75 a, 75 b}$ L. Guzzi, ${ }^{75 a, 75 b}$ M. Malberti, ${ }^{75 a}$ S. Malvezzi, ${ }^{75 a}$ A. Massironi, ${ }^{75 a}$ D. Menasce,${ }^{75 a}$ F. Monti, ${ }^{75 a, 75 b}$ L. Moroni, ${ }^{75 a}$ M. Paganoni, ${ }^{75 a, 75 b}$ D. Pedrini, ${ }^{75 a}$ S. Ragazzi, ${ }^{75 a, 75 b}$ T. Tabarelli de Fatis, ${ }^{75 a, 75 b}$ D. Valsecchi, ${ }^{75 a, 75 b, u}$ D. Zuolo, ${ }^{75 a, 75 b}$ S. Buontempo, ${ }^{76 \mathrm{a}}$ F. Carnevali, ${ }^{76 a, 76 \mathrm{~b}}$ N. Cavallo, ${ }^{76 a, 76 \mathrm{c}}$ A. De Iorio, ${ }^{76 a, 76 \mathrm{~b}}$ F. Fabozzi, ${ }^{76 a, 76 \mathrm{c}}$ A. O. M. Iorio, ${ }^{76 a, 76 \mathrm{~b}}$ L. Lista, ${ }^{76 a, 76 b}$ S. Meola, ${ }^{76 a, 76 \mathrm{~d}, \mathrm{u}}$ P. Paolucci, ${ }^{76 a, u}$ B. Rossi, ${ }^{76 \mathrm{a}}$ C. Sciacca, ${ }^{76 a, 76 \mathrm{~b}}$ P. Azzi, ${ }^{77 \mathrm{a}}$ N. Bacchetta, ${ }^{77 \mathrm{a}}$ D. Bisello, ${ }^{77 a, 77 b}$ P. Bortignon, ${ }^{77 a}$ A. Bragagnolo, ${ }^{77 a, 77 b}$ R. Carlin, ${ }^{77 a, 77 b}$ P. Checchia, ${ }^{77 a}$ P. De Castro Manzano, ${ }^{77 a}$ T. Dorigo, ${ }^{77 a}$ F. Gasparini, ${ }^{77 a, 77 b}$ U. Gasparini, ${ }^{77 a, 77 b}$ S. Y. Hoh, ${ }^{77 a, 77 b}$ L. Layer, ${ }^{77 a, t t}$ M. Margoni, ${ }^{77,77 b}$ A. T. Meneguzzo, ${ }^{77 a, 77 b}$ M. Presilla, ${ }^{77 a, 77 b}$ P. Ronchese, ${ }^{77 a, 77 b}$ R. Rossin, ${ }^{77 a, 77 b}$ F. Simonetto, ${ }^{77 a, 77 b}$ G. Strong, ${ }^{77 a}$ M. Tosi, ${ }^{77 a, 77 b}$ H. YARAR, ${ }^{77 a, 77 b}$ M. Zanetti, ${ }^{77 a, 77 b}$ P. Zotto, ${ }^{77 a, 77 b}$ A. Zucchetta, ${ }^{77 a, 77 b}$ G. Zumerle, ${ }^{77 a, 77 b}$ C. Aime ${ }^{-78 a, 78 b}$ A. Braghieri, ${ }^{78 a}$ S. Calzaferri, ${ }^{78 a, 78 b}$ D. Fiorina, ${ }^{78 a, 78 b}$ P. Montagna, ${ }^{78 a, 78 b}$ S. P. Ratti, ${ }^{78 a, 78 b}$ V. Re, ${ }^{78 a}$ M. Ressegotti, ${ }^{78 a}, 78 b$ C. Riccardi, ${ }^{78 a, 78 b}$ P. Salvini, ${ }^{78 a}$ I. Vai, ${ }^{78 a}$ P. Vitulo, ${ }^{78 a, 78 b}$ G. M. Bilei, ${ }^{79 a}$ D. Ciangottini, ${ }^{79 a, 79 b}$ L. Fanò,${ }^{79 a, 79 b}$ P. Lariccia, ${ }^{79 a, 79 b}$ G. Mantovani, ${ }^{79 a, 79 b}$ V. Mariani, ${ }^{79 a, 79 b}$ M. Menichelli, ${ }^{79 a}$ F. Moscatelli, ${ }^{79 a}$ A. Piccinelli, ${ }^{79 a}, 79 b$ A. Rossi,${ }^{79 a, 79 b}$ A. Santocchia, ${ }^{79 a, 79 b}$ D. Spiga, ${ }^{79 a}$ T. Tedeschi, ${ }^{79 a, 79 b}$ P. Azzurri, ${ }^{80 a}$ G. Bagliesi, ${ }^{80 a}$ V. Bertacchi $,{ }^{80 a}, 80 \mathrm{c}$ L. Bianchini, ${ }^{80 a}$ T. Boccali, ${ }^{80 a}$ E. Bossini, ${ }^{80 a}$ R. Castaldi, ${ }^{80 a}$

M. A. Ciocci, ${ }^{80,80 b}$ R. Dell'Orso, ${ }^{80 a}$ M. R. Di Domenico, ${ }^{80 a, 80 d}$ S. Donato, ${ }^{80 a}$ A. Giassi, ${ }^{80 a}$ M. T. Grippo, ${ }^{80 a}$ F. Ligabue, ${ }^{80 a, 80 c}$ E. Manca, ${ }^{80 \mathrm{a}, 80 \mathrm{c}}$ G. Mandorli, ${ }^{80 \mathrm{a}, 80 \mathrm{c}}$ A. Messineo, ${ }^{80 \mathrm{a}, 80 \mathrm{~b}}$ F. Palla, ${ }^{80 \mathrm{a}}$ G. Ramirez-Sanchez, ${ }^{80,80 \mathrm{c}}$ A. Rizzi, ${ }^{80 \mathrm{a}, 80 \mathrm{~b}}$

G. Rolandi, ${ }^{80 a, 80 \mathrm{c}}$ S. Roy Chowdhury, ${ }^{80 a, 80 \mathrm{c}}$ A. Scribano, ${ }^{80 \mathrm{a}}$ N. Shafiei ${ }^{80 \mathrm{a}, 80 \mathrm{~b}}$ P. Spagnolo, ${ }^{80 \mathrm{a}}$ R. Tenchini, ${ }^{80 \mathrm{a}}$ G. Tonelli, ${ }^{80 \mathrm{a}, 80 \mathrm{~b}}$ N. Turini ${ }^{80 a, 80 \mathrm{~d}}$ A. Venturi, ${ }^{80 \mathrm{a}}$ P. G. Verdini ${ }^{80 \mathrm{a}}$ F. Cavallari, ${ }^{81 \mathrm{a}}$ M. Cipriani, ${ }^{81 \mathrm{a}, 81 \mathrm{~b}}$ D. Del Re,${ }^{81 \mathrm{a}, 81 \mathrm{~b}}$ E. Di Marco, ${ }^{81 \mathrm{a}}$ M. Diemoz, ${ }^{81 \mathrm{a}}$ E. Longo,${ }^{81 \mathrm{a}, 81 \mathrm{~b}}$ P. Meridiani, ${ }^{81 \mathrm{a}}$ G. Organtini, ${ }^{81 \mathrm{a}, 81 \mathrm{~b}}$ F. Pandolfi, ${ }^{81 \mathrm{a}}$ R. Paramatti, ${ }^{81,81 \mathrm{~b}}$ C. Quaranta,${ }^{81 a, 81 b}$ S. Rahatlou, ${ }^{81 a, 81 b}$ C. Rovelli, ${ }^{81 a}$ F. Santanastasio, ${ }^{81 a, 81 b}$ L. Soffi, ${ }^{81 a, 81 b}$ R. Tramontano, ${ }^{81 a, 81 b}$ N. Amapane, ${ }^{82 a, 82 b}$ R. Arcidiacono, ${ }^{82 a, 82 c}$ S. Argiro, ${ }^{82 a, 82 b}$ M. Arneodo, ${ }^{82 a, 82 c}$ N. Bartosik, ${ }^{82 a}$ R. Bellan, ${ }^{82 a, 82 b}$ A. Bellora, ${ }^{82 a, 82 b}$ J. Berenguer Antequera, ${ }^{82 a, 82 b}$ C. Biino, ${ }^{82 a}$ A. Cappati, ${ }^{82 a, 82 b}$ N. Cartiglia, ${ }^{82 a}$ S. Cometti, ${ }^{82 a}$ M. Costa, ${ }^{82 a, 82 b}$ R. Covarelli, ${ }^{82 a, 82 b}$ N. Demaria, ${ }^{82 a}$ B. Kiani, ${ }^{82 a, 82 b}$ F. Legger, ${ }^{82 a}$ C. Mariotti, ${ }^{82 a}$ S. Maselli, ${ }^{82 a}$ E. Migliore, ${ }^{82 a, 82 b}$ V. Monaco, ${ }^{82 a, 82 b}$ E. Monteil,${ }^{82 a, 82 b}$ M. Monteno, ${ }^{82 a}$ M. M. Obertino, ${ }^{82 a, 82 b}$ G. Ortona, ${ }^{82 a}$ L. Pacher, ${ }^{82 a, 82 b}$ N. Pastrone, ${ }^{82 a}$ M. Pelliccioni, ${ }^{82 \mathrm{a}}$ G. L. Pinna Angioni, ${ }^{82 a, 82 b}$ M. Ruspa,${ }^{82 a, 82 c}$ R. Salvatico, ${ }^{82 a, 82 b}$ K. Shchelina, ${ }^{82 a, 82 b}$ F. Siviero, ${ }^{82 a, 82 b}$ V. Sola, ${ }^{82 a}$ A. Solano, ${ }^{82 a, 82 b}$ D. Soldi, ${ }^{82 a, 82 b}$ A. Staiano, ${ }^{82 a}$ M. Tornago, ${ }^{82 a, 82 b}$ D. Trocino, ${ }^{82 a, 82 b}$ S. Belforte ${ }^{83 a}$ V. Candelise, ${ }^{83 a, 83 b}$ M. Casarsa, ${ }^{83 a}$ F. Cossutti, ${ }^{83 a}$ A. Da Rold, ${ }^{83 a, 83 b}$ G. Della Ricca, ${ }^{83 a, 83 b}$ F. Vazzoler, ${ }^{83 a, 83 b}$ S. Dogra, ${ }^{84}$ C. Huh ${ }^{84}$ B. Kim ${ }^{84}$ D. H. Kim,${ }^{84}$ G. N. Kim,${ }^{84}$ J. Lee, ${ }^{84}$ S. W. Lee,${ }^{84}$ C. S. Moon, ${ }^{84}$ Y. D. Oh ${ }^{84}$ S. I. Pak, ${ }^{84}$ B. C. Radburn-Smith ${ }^{84}$ S. Sekmen, ${ }^{84}$ Y. C. Yang, ${ }^{84}$ H. Kim,${ }^{85}$ D. H. Moon ${ }^{85}$ T. J. Kim, ${ }^{86}$ J. Park, ${ }^{86}$ S. Cho, ${ }^{87}$ S. Choi, ${ }^{87}$ Y. Go, ${ }^{87}$ B. Hong, ${ }^{87}$ K. Lee, ${ }^{87}$ K. S. Lee, ${ }^{87}$ J. Lim, ${ }^{87}$ J. Park, ${ }^{87}$ S. K. Park, ${ }^{87}$ J. Yoo, ${ }^{87}$ J. Goh, ${ }^{88}$ A. Gurtu, ${ }^{88}$ H. S. Kim, ${ }^{89}$ Y. Kim, ${ }^{89}$ J. Almond,${ }^{90}$ J. H. Bhyun, ${ }^{90}$ J. Choi, ${ }^{90}$ S. Jeon, ${ }^{90}$ J. Kim, ${ }^{90}$ J. S. Kim, ${ }^{90}$ S. Ko, ${ }^{90}$ H. Kwon, ${ }^{90}$ H. Lee, ${ }^{90}$ S. Lee, ${ }^{90}$ B. H. Oh, ${ }^{90}$ M. Oh ${ }^{90}$ S. B. Oh ${ }^{90}$ H. Seo, ${ }^{90}$ U. K. Yang, ${ }^{90}$ I. Yoon, ${ }^{90}$ D. Jeon,${ }^{91}$ J. H. Kim,${ }^{91}$ B. Ko, ${ }^{91}$ J. S. H. Lee, ${ }^{91}$ I. C. Park, ${ }^{91}$ Y. Roh, ${ }^{91}$ D. Song, ${ }^{91}$ I. J. Watson, ${ }^{91}$ S. Ha,${ }^{92}$ H. D. Yoo, ${ }^{92}$ Y. Choi, ${ }^{93}$ Y. Jeong, ${ }^{93}$ H. Lee,${ }^{93}$ Y. Lee,${ }^{93}$ I. Yu,${ }^{93}$ T. Beyrouthy, ${ }^{94}$ Y. Maghrbi,${ }^{94}$ V. Veckalns, ${ }^{95, \text { иu }}$ M. Ambrozas, ${ }^{96}$ A. Juodagalvis, ${ }^{96}$ A. Rinkevicius, ${ }^{96}$ G. Tamulaitis, ${ }^{96}$ A. Vaitkevicius, ${ }^{96}$ W. A. T. Wan Abdullah, ${ }^{97}$ M. N. Yusli, ${ }^{97}$ Z. Zolkapli, ${ }^{97}$ J. F. Benitez,${ }^{98}$ A. Castaneda Hernandez,${ }^{98}$ J. A. Murillo Quijada, ${ }^{98}$ L. Valencia Palomo, ${ }^{98}$ G. Ayala, ${ }^{99}$ H. Castilla-Valdez, ${ }^{99}$ E. De La Cruz-Burelo, ${ }^{99}$ I. Heredia-De La Cruz, ${ }^{99, v v}$ R. Lopez-Fernandez ${ }^{99}$ C. A. Mondragon Herrera, ${ }^{99}$ D. A. Perez Navarro, ${ }^{99}$

A. Sanchez-Hernandez, ${ }^{99}$ S. Carrillo Moreno, ${ }^{100}$ C. Oropeza Barrera, ${ }^{100}$ M. Ramirez-Garcia, ${ }^{100}$ F. Vazquez Valencia, ${ }^{100}$ I. Pedraza, ${ }^{101}$ H. A. Salazar Ibarguen, ${ }^{101}$ C. Uribe Estrada, ${ }^{101}$ J. Mijuskovic, ${ }^{102, w w}$ N. Raicevic, ${ }^{102}$ D. Krofcheck, ${ }^{103}$ S. Bheesette, ${ }^{104}$ P. H. Butler, ${ }^{104}$ A. Ahmad, ${ }^{105}$ M. I. Asghar, ${ }^{105}$ A. Awais, ${ }^{105}$ M. I. M. Awan,${ }^{105}$ H. R. Hoorani, ${ }^{105}$ W. A. Khan, ${ }^{105}$ M. A. Shah, ${ }^{105}$ M. Shoaib, ${ }^{105}$ M. Waqas, ${ }^{105}$ V. Avati, ${ }^{106}$ L. Grzanka, ${ }^{106}$ M. Malawski, ${ }^{106}$ H. Bialkowska, ${ }^{107}$ M. Bluj, ${ }^{107}$ B. Boimska, ${ }^{107}$ T. Frueboes, ${ }^{107}$ M. Górski, ${ }^{107}$ M. Kazana, ${ }^{107}$ M. Szleper, ${ }^{107}$ P. Traczyk ${ }^{107}$ P. Zalewski, ${ }^{107}$ K. Bunkowski, ${ }^{108}$ K. Doroba, ${ }^{108}$ A. Kalinowski, ${ }^{108}$ M. Konecki, ${ }^{108}$ J. Krolikowski, ${ }^{108}$ M. Walczak, ${ }^{108}$ M. Araujo, ${ }^{109}$ P. Bargassa, ${ }^{109}$ D. Bastos, ${ }^{109}$ A. Boletti, ${ }^{109}$ P. Faccioli, ${ }^{109}$ M. Gallinaro, ${ }^{109}$ J. Hollar, ${ }^{109}$ N. Leonardo, ${ }^{109}$ T. Niknejad, ${ }^{109}$ 
J. Seixas, ${ }^{109}$ O. Toldaiev, ${ }^{109}$ J. Varela, ${ }^{109}$ S. Afanasiev, ${ }^{110}$ D. Budkouski, ${ }^{110}$ P. Bunin,${ }^{110}$ M. Gavrilenko, ${ }^{110}$ I. Golutvin, ${ }^{110}$ I. Gorbunov, ${ }^{110}$ A. Kamenev, ${ }^{110}$ V. Karjavine, ${ }^{110}$ A. Lanev, ${ }^{110}$ A. Malakhov, ${ }^{110}$ V. Matveev, ${ }^{11, x x, y y}$ V. Palichik, ${ }^{110}$ V. Perelygin, ${ }^{110}$ M. Savina, ${ }^{110}$ D. Seitova, ${ }^{110}$ V. Shalaev, ${ }^{110}$ S. Shmatov, ${ }^{110}$ S. Shulha, ${ }^{110}$ V. Smirnov, ${ }^{110}$ O. Teryaev, ${ }^{110}$ N. Voytishin, ${ }^{110}$ A. Zarubin, ${ }^{110}$ I. Zhizhin, ${ }^{110}$ G. Gavrilov, ${ }^{111}$ V. Golovtcov, ${ }^{111}$ Y. Ivanov, ${ }^{111}$ V. Kim, ${ }^{11, z z}$ E. Kuznetsova, ${ }^{111, \text { aaa }}$ V. Murzin, ${ }^{111}$ V. Oreshkin, ${ }^{111}$ I. Smirnov, ${ }^{111}$ D. Sosnov, ${ }^{111}$ V. Sulimov, ${ }^{111}$ L. Uvarov, ${ }^{111}$ S. Volkov, ${ }^{111}$ A. Vorobyev, ${ }^{111}$ Yu. Andreev, ${ }^{112}$ A. Dermenev, ${ }^{112}$ S. Gninenko, ${ }^{112}$ N. Golubev, ${ }^{112}$ A. Karneyeu, ${ }^{112}$ M. Kirsanov, ${ }^{112}$ N. Krasnikov, ${ }^{112}$ A. Pashenkov, ${ }^{112}$ G. Pivovarov, ${ }^{112}$ D. Tlisov, ${ }^{112, a}$ A. Toropin, ${ }^{112}$ V. Epshteyn, ${ }^{113}$ V. Gavrilov, ${ }^{113}$ N. Lychkovskaya, ${ }^{113}$ A. Nikitenko, ${ }^{113, \text { bbb }}$ V. Popov, ${ }^{113}$ G. Safronov, ${ }^{113}$ A. Spiridonov ${ }^{113}$ A. Stepennov, ${ }^{113}$ M. Toms, ${ }^{113}$ E. Vlasov, ${ }^{113}$ A. Zhokin, ${ }^{113}$ T. Aushev, ${ }^{114}$ R. Chistov, ${ }^{115, \text { ccc }}$ M. Danilov, ${ }^{115, \text { ddd }}$ A. Oskin, ${ }^{115}$ P. Parygin, ${ }^{115}$ S. Polikarpov, ${ }^{115, \text { ccc }}$ V. Andreev, ${ }^{116}$ M. Azarkin, ${ }^{116}$ I. Dremin, ${ }^{116}$ M. Kirakosyan, ${ }^{116}$ A. Terkulov, ${ }^{116}$ A. Belyaev, ${ }^{117}$ E. Boos, ${ }^{117}$ M. Dubinin,,${ }^{17, \text {,eee }}$ L. Dudko, ${ }^{117}$ A. Ershov, ${ }^{117}$ A. Gribushin, ${ }^{117}$ V. Klyukhin, ${ }^{117}$ O. Kodolova, ${ }^{117}$ I. Lokhtin, ${ }^{117}$ S. Obraztsov, ${ }^{117}$ S. Petrushanko, ${ }^{117}$ V. Savrin, ${ }^{117}$ A. Snigirev, ${ }^{117}$ V. Blinov, ${ }^{118, \text { fff }}$ T. Dimova, ${ }^{118, \text { fff }}$ L. Kardapoltsev, ${ }^{118, f f f}$ I. Ovtin, ${ }^{18, \text { fff }}$ Y. Skovpen, ${ }^{118, \text { fff }}$ I. Azhgirey, ${ }^{119}$ I. Bayshev, ${ }^{119}$ V. Kachanov, ${ }^{119}$ A. Kalinin, ${ }^{119}$ D. Konstantinov, ${ }^{119}$ V. Petrov,${ }^{119}$ R. Ryutin, ${ }^{119}$ A. Sobol, ${ }^{119}$ S. Troshin, ${ }^{119}$ N. Tyurin, ${ }^{119}$ A. Uzunian, ${ }^{119}$ A. Volkov, ${ }^{119}$ A. Babaev, ${ }^{120}$ V. Okhotnikov, ${ }^{120}$ L. Sukhikh, ${ }^{120}$ V. Borchsh, ${ }^{121}$ V. Ivanchenko, ${ }^{121}$ E. Tcherniaev, ${ }^{121}$ P. Adzic, ${ }^{122, g g g}$ M. Dordevic, ${ }^{122}$ P. Milenovic, ${ }^{122}$ J. Milosevic, ${ }^{122}$ V. Milosevic, ${ }^{122}$ M. Aguilar-Benitez, ${ }^{123}$ J. Alcaraz Maestre, ${ }^{123}$ A. Álvarez Fernández, ${ }^{123}$ I. Bachiller, ${ }^{123}$ M. Barrio Luna, ${ }^{123}$ Cristina F. Bedoya, ${ }^{123}$ C. A. Carrillo Montoya ${ }^{123}$ M. Cepeda,${ }^{123}$ M. Cerrada, ${ }^{123}$ N. Colino, ${ }^{123}$ B. De La Cruz, ${ }^{123}$

A. Delgado Peris, ${ }^{123}$ J. P. Fernández Ramos, ${ }^{123}$ J. Flix, ${ }^{123}$ M. C. Fouz, ${ }^{123}$ O. Gonzalez Lopez, ${ }^{123}$ S. Goy Lopez, ${ }^{123}$ J. M. Hernandez, ${ }^{123}$ M. I. Josa, ${ }^{123}$ J. León Holgado, ${ }^{123}$ D. Moran, ${ }^{123}$ Á. Navarro Tobar, ${ }^{123}$ A. Pérez-Calero Yzquierdo, ${ }^{123}$ J. Puerta Pelayo, ${ }^{123}$ I. Redondo, ${ }^{123}$ L. Romero, ${ }^{123}$ S. Sánchez Navas, ${ }^{123}$ M. S. Soares, ${ }^{123}$ L. Urda Gómez, ${ }^{123}$ C. Willmott, ${ }^{123}$ J. F. de Trocóniz, ${ }^{124}$ R. Reyes-Almanza, ${ }^{124}$ B. Alvarez Gonzalez, ${ }^{125}$ J. Cuevas, ${ }^{125}$ C. Erice, ${ }^{125}$ J. Fernandez Menendez, ${ }^{125}$ S. Folgueras, ${ }^{125}$ I. Gonzalez Caballero, ${ }^{125}$ E. Palencia Cortezon, ${ }^{125}$ C. Ramón Álvarez, ${ }^{125}$ J. Ripoll Sau, ${ }^{125}$

V. Rodríguez Bouza, ${ }^{125}$ A. Trapote, ${ }^{125}$ J. A. Brochero Cifuentes, ${ }^{126}$ I. J. Cabrillo, ${ }^{126}$ A. Calderon, ${ }^{126}$ B. Chazin Quero, ${ }^{126}$ J. Duarte Campderros, ${ }^{126}$ M. Fernandez, ${ }^{126}$ C. Fernandez Madrazo, ${ }^{126}$ P. J. Fernández Manteca, ${ }^{126}$ A. García Alonso, ${ }^{126}$ G. Gomez, ${ }^{126}$ C. Martinez Rivero, ${ }^{126}$ P. Martinez Ruiz del Arbol, ${ }^{126}$ F. Matorras, ${ }^{126}$ J. Piedra Gomez, ${ }^{126}$ C. Prieels, ${ }^{126}$ F. Ricci-Tam, ${ }^{126}$ T. Rodrigo, ${ }^{126}$ A. Ruiz-Jimeno, ${ }^{126}$ L. Scodellaro, ${ }^{126}$ N. Trevisani, ${ }^{126}$ I. Vila, ${ }^{126}$ J. M. Vizan Garcia, ${ }^{126}$ MK Jayananda, ${ }^{127}$ B. Kailasapathy, ${ }^{127, h h h}$ D. U. J. Sonnadara, ${ }^{127}$ DDC Wickramarathna, ${ }^{127}$ W. G. D. Dharmaratna, ${ }^{128}$

K. Liyanage ${ }^{128}$ N. Perera, ${ }^{128}$ N. Wickramage, ${ }^{128}$ T. K. Aarrestad, ${ }^{129}$ D. Abbaneo, ${ }^{129}$ J. Alimena ${ }^{129}$ E. Auffray, ${ }^{129}$ G. Auzinger, ${ }^{129}$ J. Baechler, ${ }^{129}$ P. Baillon, ${ }^{129, a}$ A. H. Ball, ${ }^{129}$ D. Barney, ${ }^{129}$ J. Bendavid, ${ }^{129}$ N. Beni, ${ }^{129}$ M. Bianco, ${ }^{129}$ A. Bocci, ${ }^{129}$ E. Brondolin, ${ }^{129}$ T. Camporesi, ${ }^{129}$ M. Capeans Garrido, ${ }^{129}$ G. Cerminara, ${ }^{129}$ S. S. Chhibra, ${ }^{129}$ L. Cristella, ${ }^{129}$ D. d'Enterria, ${ }^{129}$ A. Dabrowski, ${ }^{129}$ N. Daci, ${ }^{129}$ A. David, ${ }^{129}$ A. De Roeck, ${ }^{129}$ M. Deile, ${ }^{129}$ R. Di Maria, ${ }^{129}$ M. Dobson, ${ }^{129}$ M. Dünser, ${ }^{129}$ N. Dupont, ${ }^{129}$ A. Elliott-Peisert, ${ }^{129}$ N. Emriskova, ${ }^{129}$ F. Fallavollita, ${ }^{129, i i i}$ D. Fasanella, ${ }^{129}$ S. Fiorendi, ${ }^{129}$ A. Florent ${ }^{129}$ G. Franzoni, ${ }^{129}$ J. Fulcher, ${ }^{129}$ W. Funk, ${ }^{129}$ S. Giani, ${ }^{129}$ D. Gigi, ${ }^{129}$ K. Gill, ${ }^{129}$ F. Glege,${ }^{129}$ L. Gouskos,${ }^{129}$ M. Haranko, ${ }^{129}$ J. Hegeman, ${ }^{129}$ Y. Iiyama, ${ }^{129}$ V. Innocente, ${ }^{129}$ T. James, ${ }^{129}$ P. Janot, ${ }^{129}$ J. Kaspar, ${ }^{129}$ J. Kieseler, ${ }^{129}$ M. Komm, ${ }^{129}$ N. Kratochwil, ${ }^{129}$ C. Lange, ${ }^{129}$ S. Laurila, ${ }^{129}$ P. Lecoq,${ }^{129}$ K. Long, ${ }^{129}$ C. Lourenço, ${ }^{129}$ L. Malgeri, ${ }^{129}$ S. Mallios, ${ }^{129}$ M. Mannelli, ${ }^{129}$ F. Meijers, ${ }^{129}$ S. Mersi, ${ }^{129}$ E. Meschi, ${ }^{129}$ F. Moortgat, ${ }^{129}$ M. Mulders, ${ }^{129}$ S. Orfanelli, ${ }^{129}$ L. Orsini, ${ }^{129}$ F. Pantaleo, ${ }^{129}$ L. Pape, ${ }^{129}$ E. Perez, ${ }^{129}$ M. Peruzzi,${ }^{129}$ A. Petrilli, ${ }^{129}$ G. Petrucciani, ${ }^{129}$ A. Pfeiffer, ${ }^{129}$ M. Pierini, ${ }^{129}$ M. Pitt, ${ }^{129}$ H. Qu, ${ }^{129}$ T. Quast, ${ }^{129}$ D. Rabady, ${ }^{129}$ A. Racz, ${ }^{129}$ M. Rieger, ${ }^{129}$ M. Rovere,${ }^{129}$ H. Sakulin, ${ }^{129}$ J. Salfeld-Nebgen, ${ }^{129}$ S. Scarfi, ${ }^{129}$ C. Schäfer, ${ }^{129}$ C. Schwick, ${ }^{129}$ M. Selvaggi, ${ }^{129}$ A. Sharma, ${ }^{129}$ P. Silva, ${ }^{129}$ W. Snoeys, ${ }^{129}$ P. Sphicas, ${ }^{129, j j j}$ S. Summers, ${ }^{129}$ V. R. Tavolaro, ${ }^{129}$ D. Treille, ${ }^{129}$ A. Tsirou, ${ }^{129}$ G. P. Van Onsem, ${ }^{129}$ M. Verzetti, ${ }^{129}$ K. A. Wozniak, ${ }^{129}$ W. D. Zeuner, ${ }^{129}$ L. Caminada,${ }^{130, k k k}$ A. Ebrahimi, ${ }^{130}$ W. Erdmann, ${ }^{130}$ R. Horisberger, ${ }^{130}$ Q. Ingram, ${ }^{130}$ H. C. Kaestli, ${ }^{130}$ D. Kotlinski, ${ }^{130}$ U. Langenegger, ${ }^{130}$ M. Missiroli, ${ }^{130}$ T. Rohe,${ }^{130}$ K. Androsov, ${ }^{131,111}$ M. Backhaus, ${ }^{131}$ P. Berger ${ }^{131}$ A. Calandri, ${ }^{131}$ N. Chernyavskaya, ${ }^{131}$ A. De Cosa, ${ }^{131}$ G. Dissertori, ${ }^{131}$ M. Dittmar,${ }^{131}$ M. Donegà, ${ }^{131}$ C. Dorfer, ${ }^{131}$ T. Gadek, ${ }^{131}$ T. A. Gómez Espinosa, ${ }^{131}$ C. Grab ${ }^{131}$ D. Hits, ${ }^{131}$ W. Lustermann, ${ }^{131}$ A.-M. Lyon, ${ }^{131}$ R. A. Manzoni, ${ }^{131}$ C. Martin Perez, ${ }^{131}$ M. T. Meinhard,${ }^{131}$ F. Micheli, ${ }^{131}$ F. Nessi-Tedaldi, ${ }^{131}$ J. Niedziela, ${ }^{131}$ F. Pauss, ${ }^{131}$ V. Perovic, ${ }^{131}$ G. Perrin, ${ }^{131}$ S. Pigazzini, ${ }^{131}$ M. G. Ratti, ${ }^{131}$ M. Reichmann, ${ }^{131}$ C. Reissel, ${ }^{131}$ T. Reitenspiess, ${ }^{131}$ B. Ristic, ${ }^{131}$ D. Ruini, ${ }^{131}$ D. A. Sanz Becerra ${ }^{131}$ M. Schönenberger, ${ }^{131}$ V. Stampf,${ }^{131}$ J. Steggemann, ${ }^{131,111}$ R. Wallny, ${ }^{131}$ D. H. Zhu, ${ }^{131}$ C. Amsler, ${ }^{132, \mathrm{mmm}}$ C. Botta, ${ }^{132}$ D. Brzhechko, ${ }^{132}$ M. F. Canelli, ${ }^{132}$ A. De Wit, ${ }^{132}$ R. Del Burgo, ${ }^{132}$ J. K. Heikkilä, ${ }^{132}$ M. Huwiler, ${ }^{132}$ A. Jofrehei, ${ }^{132}$ B. Kilminster, ${ }^{132}$ S. Leontsinis, ${ }^{132}$ A. Macchiolo, ${ }^{132}$ P. Meiring, ${ }^{132}$ V. M. Mikuni, ${ }^{132}$ 
U. Molinatti, ${ }^{132}$ I. Neutelings, ${ }^{132}$ G. Rauco, ${ }^{132}$ A. Reimers, ${ }^{132}$ P. Robmann, ${ }^{132}$ S. Sanchez Cruz, ${ }^{132}$ K. Schweiger, ${ }^{132}$ Y. Takahashi, ${ }^{132}$ C. Adloff, ${ }^{133, \text { nnn }}$ C. M. Kuo, ${ }^{133}$ W. Lin, ${ }^{133}$ A. Roy, ${ }^{133}$ T. Sarkar, ${ }^{133,11}$ S. S. Yu, ${ }^{133}$ L. Ceard, ${ }^{134}$ P. Chang, ${ }^{134}$ Y. Chao, ${ }^{134}$ K. F. Chen, ${ }^{134}$ P. H. Chen, ${ }^{134}$ W.-S. Hou, ${ }^{134}$ Y. y. Li, ${ }^{134}$ R.-S. Lu, ${ }^{134}$ E. Paganis, ${ }^{134}$ A. Psallidas, ${ }^{134}$ A. Steen, ${ }^{134}$ E. Yazgan, ${ }^{134}$ P. r. Yu, ${ }^{134}$ B. Asavapibhop, ${ }^{135}$ C. Asawatangtrakuldee, ${ }^{135}$ N. Srimanobhas, ${ }^{135}$ M. N. Bakirci, ${ }^{136,000}$ F. Boran, ${ }^{136}$ S. Damarseckin, ${ }^{136, p p p}$ Z. S. Demiroglu, ${ }^{136}$ F. Dolek, ${ }^{136}$ I. Dumanoglu, ${ }^{136, q q q}$ G. Gokbulut, ${ }^{136}$ Y. Guler, ${ }^{136}$ E. Gurpinar Guler, ${ }^{136, \text { rrr }}$ I. Hos, ${ }^{136, \text { sss }}$ C. Isik, ${ }^{136}$ E. E. Kangal, ${ }^{136, t \text { tt }}$ O. Kara, ${ }^{136}$ A. Kayis Topaksu, ${ }^{136}$ U. Kiminsu, ${ }^{136}$ G. Onengut, ${ }^{136}$ K. Ozdemir, ${ }^{136, \text { uuu }}$ A. E. Simsek, ${ }^{136}$ B. Tali, ${ }^{136, \text { vvv }}$ U. G. Tok, ${ }^{136}$ H. Topakli, ${ }^{136, w w w}$ S. Turkcapar, ${ }^{136}$ I. S. Zorbakir, ${ }^{136}$ C. Zorbilmez, ${ }^{136}$ B. Isildak, ${ }^{137, \text { xxx }}$ G. Karapinar, ${ }^{137, y y y}$ K. Ocalan, ${ }^{137, \text { zzz }}$ M. Yalvac, ${ }^{137, \text { aaaa }}$ B. Akgun, ${ }^{138}$ I. O. Atakisi, ${ }^{138}$ E. Gülmez, ${ }^{138}$ M. Kaya,${ }^{138, b b b b}$ O. Kaya,${ }^{138, \text { cccc }}$ Ö. Özçelik, ${ }^{138}$ S. Tekten, ${ }^{138, \text { dddd }}$ E. A. Yetkin, ${ }^{138, \text { eeee }}$ A. Cakir, ${ }^{139}$ K. Cankocak, ${ }^{139, q q q}$ Y. Komurcu, ${ }^{139}$ S. Sen, ${ }^{139, f f f r}$ F. Aydogmus Sen, ${ }^{140}$ S. Cerci, ${ }^{140, v v v}$ B. Kaynak, ${ }^{140}$ S. Ozkorucuklu, ${ }^{140}$ D. Sunar Cerci, ${ }^{140, v v v}$ B. Grynyov, ${ }^{141}$ L. Levchuk, ${ }^{142}$ E. Bhal, ${ }^{143}$ S. Bologna, ${ }^{143}$ J. J. Brooke, ${ }^{143}$ A. Bundock ${ }^{143}$ E. Clement, ${ }^{143}$ D. Cussans, ${ }^{143}$ H. Flacher, ${ }^{143}$ J. Goldstein,${ }^{143}$ G. P. Heath ${ }^{143}$ H. F. Heath, ${ }^{143}$ L. Kreczko, ${ }^{143}$ B. Krikler, ${ }^{143}$ S. Paramesvaran, ${ }^{143}$ T. Sakuma, ${ }^{143}$ S. Seif El Nasr-Storey, ${ }^{143}$ V. J. Smith, ${ }^{143}$ N. Stylianou, ${ }^{143, g g g g}$ J. Taylor, ${ }^{143}$ A. Titterton, ${ }^{143}$ K. W. Bell, ${ }^{144}$ A. Belyaev, ${ }^{144, h h h h}$ C. Brew,${ }^{144}$ R. M. Brown, ${ }^{144}$ D. J. A. Cockerill, ${ }^{144}$ K. V. Ellis, ${ }^{144}$ K. Harder, ${ }^{144}$ S. Harper,${ }^{144}$ J. Linacre, ${ }^{144}$ K. Manolopoulos, ${ }^{144}$ D. M. Newbold, ${ }^{144}$ E. Olaiya, ${ }^{144}$ D. Petyt, ${ }^{144}$ T. Reis, ${ }^{144}$ T. Schuh, ${ }^{144}$ C. H. Shepherd-Themistocleous, ${ }^{144}$ A. Thea,${ }^{144}$ I. R. Tomalin, ${ }^{144}$ T. Williams, ${ }^{144}$ R. Bainbridge, ${ }^{145}$ P. Bloch, ${ }^{145}$ S. Bonomally, ${ }^{145}$ J. Borg, ${ }^{145}$ S. Breeze, ${ }^{145}$ O. Buchmuller, ${ }^{145}$ V. Cepaitis, ${ }^{145}$ G. S. Chahal,,${ }^{145, \text { iiii }}$ D. Colling,,${ }^{145}$ P. Dauncey, ${ }^{145}$ G. Davies, ${ }^{145}$ M. Della Negra, ${ }^{145}$ S. Fayer, ${ }^{145}$ G. Fedi, ${ }^{145}$ G. Hall ${ }^{145}$ M. H. Hassanshahi, ${ }^{145}$ G. Iles, ${ }^{145}$ J. Langford, ${ }^{145}$ L. Lyons, ${ }^{145}$ A.-M. Magnan, ${ }^{145}$ S. Malik, ${ }^{145}$ A. Martelli, ${ }^{145}$ J. Nash $,{ }^{145}, \mathrm{jjj}$ V. Palladino, ${ }^{145}$ M. Pesaresi, ${ }^{145}$ D. M. Raymond, ${ }^{145}$ A. Richards, ${ }^{145}$ A. Rose, ${ }^{145}$ E. Scott, ${ }^{145}$ C. Seez, ${ }^{145}$ A. Shtipliyski, ${ }^{145}$ A. Tapper, ${ }^{145}$ K. Uchida, ${ }^{145}$ T. Virdee, ${ }^{145, u}$ N. Wardle, ${ }^{145}$ S. N. Webb, ${ }^{145}$ D. Winterbottom, ${ }^{145}$ A. G. Zecchinelli, ${ }^{145}$ J. E. Cole, ${ }^{146}$ A. Khan, ${ }^{146}$ P. Kyberd, ${ }^{146}$ C. K. Mackay, ${ }^{146}$

I. D. Reid, ${ }^{146}$ L. Teodorescu, ${ }^{146}$ S. Zahid, ${ }^{146}$ S. Abdullin, ${ }^{147}$ A. Brinkerhoff, ${ }^{147}$ B. Caraway, ${ }^{147}$ J. Dittmann, ${ }^{147}$ K. Hatakeyama, ${ }^{147}$ A. R. Kanuganti, ${ }^{147}$ B. McMaster, ${ }^{147}$ N. Pastika, ${ }^{147}$ S. Sawant,${ }^{147}$ C. Smith, ${ }^{147}$ C. Sutantawibul, ${ }^{147}$ J. Wilson, ${ }^{147}$ R. Bartek, ${ }^{148}$ A. Dominguez, ${ }^{148}$ R. Uniyal, ${ }^{148}$ A. M. Vargas Hernandez, ${ }^{148}$ A. Buccilli, ${ }^{149}$ O. Charaf, ${ }^{149}$ S. I. Cooper, ${ }^{149}$ D. Di Croce, ${ }^{149}$ S. V. Gleyzer, ${ }^{149}$ C. Henderson, ${ }^{149}$ C. U. Perez, ${ }^{149}$ P. Rumerio, ${ }^{149}$ C. West, ${ }^{149}$ A. Akpinar, ${ }^{150}$ A. Albert, ${ }^{150}$ D. Arcaro, ${ }^{150}$ C. Cosby, ${ }^{150}$ Z. Demiragli, ${ }^{150}$ D. Gastler, ${ }^{150}$ J. Rohlf, ${ }^{150}$ K. Salyer, ${ }^{150}$ D. Sperka, ${ }^{150}$ D. Spitzbart, ${ }^{150}$ I. Suarez, ${ }^{150}$ S. Yuan, ${ }^{150}$ D. Zou, ${ }^{150}$ G. Benelli, ${ }^{151}$ B. Burkle, ${ }^{151}$ X. Coubez, ${ }^{151, v}$ D. Cutts, ${ }^{151}$ Y. t. Duh, ${ }^{151}$ M. Hadley ${ }^{151}$ U. Heintz, ${ }^{151}$ J. M. Hogan, ${ }^{151, k k k k}$ K. H. M. Kwok,${ }^{151}$ E. Laird, ${ }^{151}$ G. Landsberg, ${ }^{151}$ K. T. Lau, ${ }^{151}$ J. Lee ${ }^{151}$ J. Luo, ${ }^{151}$ M. Narain, ${ }^{151}$ S. Sagir, ${ }^{151,1111}$ E. Usai, ${ }^{151}$ W. Y. Wong, ${ }^{151}$ X. Yan ${ }^{151}$ D. Yu, ${ }^{151}$ W. Zhang, ${ }^{151}$ C. Brainerd ${ }^{152}$ R. Breedon, ${ }^{152}$ M. Calderon De La Barca Sanchez, ${ }^{152}$ M. Chertok, ${ }^{152}$ J. Conway, ${ }^{152}$ P. T. Cox, ${ }^{152}$ R. Erbacher, ${ }^{152}$ F. Jensen, ${ }^{152}$ O. Kukral, ${ }^{152}$ R. Lander, ${ }^{152}$ M. Mulhearn, ${ }^{152}$ D. Pellett, ${ }^{152}$ D. Taylor, ${ }^{152}$ M. Tripathi, ${ }^{152}$ Y. Yao, ${ }^{152}$ F. Zhang, ${ }^{152}$ M. Bachtis, ${ }^{153}$ R. Cousins, ${ }^{153}$ A. Dasgupta, ${ }^{153}$ A. Datta, ${ }^{153}$ D. Hamilton, ${ }^{153}$ J. Hauser, ${ }^{153}$ M. Ignatenko, ${ }^{153}$ M. A. Iqbal, ${ }^{153}$

T. Lam, ${ }^{153}$ N. Mccoll, ${ }^{153}$ W. A. Nash, ${ }^{153}$ S. Regnard, ${ }^{153}$ D. Saltzberg, ${ }^{153}$ C. Schnaible, ${ }^{153}$ B. Stone, ${ }^{153}$ V. Valuev, ${ }^{153}$

K. Burt, ${ }^{154}$ Y. Chen, ${ }^{154}$ R. Clare, ${ }^{154}$ J. W. Gary, ${ }^{154}$ G. Hanson, ${ }^{154}$ G. Karapostoli, ${ }^{154}$ O. R. Long, ${ }^{154}$ N. Manganelli, ${ }^{154}$ M. Olmedo Negrete, ${ }^{154}$ W. Si, ${ }^{154}$ S. Wimpenny, ${ }^{154}$ Y. Zhang, ${ }^{154}$ J. G. Branson, ${ }^{155}$ P. Chang, ${ }^{155}$ S. Cittolin, ${ }^{155}$

S. Cooperstein, ${ }^{155}$ N. Deelen, ${ }^{155}$ J. Duarte, ${ }^{155}$ R. Gerosa, ${ }^{155}$ L. Giannini, ${ }^{155}$ D. Gilbert, ${ }^{155}$ J. Guiang, ${ }^{155}$ R. Kansal, ${ }^{155}$ V. Krutelyov, ${ }^{155}$ R. Lee, ${ }^{155}$ J. Letts, ${ }^{155}$ M. Masciovecchio, ${ }^{155}$ S. May, ${ }^{155}$ S. Padhi, ${ }^{155}$ M. Pieri, ${ }^{155}$ B. V. Sathia Narayanan, ${ }^{155}$ V. Sharma, ${ }^{155}$ M. Tadel, ${ }^{155}$ A. Vartak, ${ }^{155}$ F. Würthwein, ${ }^{155}$ Y. Xiang, ${ }^{155}$ A. Yagil, ${ }^{155}$ N. Amin, ${ }^{156}$ C. Campagnari, ${ }^{156}$ M. Citron, ${ }^{156}$ A. Dorsett, ${ }^{156}$ V. Dutta, ${ }^{156}$ J. Incandela, ${ }^{156}$ M. Kilpatrick,${ }^{156}$ B. Marsh, ${ }^{156}$ H. Mei, ${ }^{156}$ A. Ovcharova, ${ }^{156}$ M. Quinnan, ${ }^{156}$ J. Richman, ${ }^{156}$ U. Sarica, ${ }^{156}$ D. Stuart, ${ }^{156}$ S. Wang, ${ }^{156}$ A. Bornheim, ${ }^{157}$ O. Cerri, ${ }^{157}$ I. Dutta, ${ }^{157}$ J. M. Lawhorn, ${ }^{157}$ N. Lu, ${ }^{157}$ J. Mao, ${ }^{157}$ H. B. Newman, ${ }^{157}$ J. Ngadiuba, ${ }^{157}$ T. Q. Nguyen, ${ }^{157}$ M. Spiropulu, ${ }^{157}$ J. R. Vlimant, ${ }^{157}$ C. Wang, ${ }^{157}$ S. Xie, ${ }^{157}$ Z. Zhang, ${ }^{157}$ R. Y. Zhu, ${ }^{157}$ J. Alison, ${ }^{158}$ M. B. Andrews, ${ }^{158}$ T. Ferguson, ${ }^{158}$ T. Mudholkar, ${ }^{158}$ M. Paulini, ${ }^{158}$ I. Vorobiev, ${ }^{158}$ J. P. Cumalat, ${ }^{159}$ W. T. Ford, ${ }^{159}$ E. MacDonald, ${ }^{159}$ R. Patel, ${ }^{159}$ A. Perloff, ${ }^{159}$ K. Stenson, ${ }^{159}$ K. A. Ulmer, ${ }^{159}$ S. R. Wagner, ${ }^{159}$ J. Alexander, ${ }^{160}$ Y. Cheng, ${ }^{160}$ J. Chu ${ }^{160}$ D. J. Cranshaw, ${ }^{160}$ K. Mcdermott, ${ }^{160}$ J. Monroy, ${ }^{160}$ J. R. Patterson, ${ }^{160}$ D. Quach, ${ }^{160}$ A. Ryd ${ }^{160}$ W. Sun, ${ }^{160}$ S. M. Tan, ${ }^{160}$ Z. Tao, ${ }^{160}$ J. Thom, ${ }^{160}$ P. Wittich, ${ }^{160}$ M. Zientek, ${ }^{160}$ M. Albrow, ${ }^{161}$ M. Alyari, ${ }^{161}$ G. Apollinari, ${ }^{161}$ A. Apresyan, ${ }^{161}$ A. Apyan, ${ }^{161}$ S. Banerjee,${ }^{161}$ L. A. T. Bauerdick, ${ }^{161}$ A. Beretvas, ${ }^{161}$ D. Berry, ${ }^{161}$ J. Berryhill, ${ }^{161}$ P. C. Bhat ${ }^{161}$ K. Burkett, ${ }^{161}$ J. N. Butler, ${ }^{161}$ A. Canepa, ${ }^{161}$ G. B. Cerati, ${ }^{161}$ H. W. K. Cheung, ${ }^{161}$ F. Chlebana, ${ }^{161}$ M. Cremonesi, ${ }^{161}$ K. F. Di Petrillo, ${ }^{161}$ V. D. Elvira, ${ }^{161}$ J. Freeman, ${ }^{161}$ Z. Gecse, ${ }^{161}$ L. Gray, ${ }^{161}$ D. Green, ${ }^{161}$ S. Grünendahl, ${ }^{161}$ O. Gutsche, ${ }^{161}$ R. M. Harris, ${ }^{161}$ R. Heller, ${ }^{161}$ T. C. Herwig, ${ }^{161}$ 
J. Hirschauer, ${ }^{161}$ B. Jayatilaka, ${ }^{161}$ S. Jindariani, ${ }^{161}$ M. Johnson, ${ }^{161}$ U. Joshi, ${ }^{161}$ P. Klabbers, ${ }^{161}$ T. Klijnsma, ${ }^{161}$ B. Klima, ${ }^{161}$ M. J. Kortelainen, ${ }^{161}$ S. Lammel, ${ }^{161}$ D. Lincoln, ${ }^{161}$ R. Lipton, ${ }^{161}$ T. Liu, ${ }^{161}$ J. Lykken, ${ }^{161}$ C. Madrid, ${ }^{161}$ K. Maeshima, ${ }^{161}$ C. Mantilla, ${ }^{161}$ D. Mason, ${ }^{161}$ P. McBride, ${ }^{161}$ P. Merkel, ${ }^{161}$ S. Mrenna, ${ }^{161}$ S. Nahn, ${ }^{161}$ V. O’Dell, ${ }^{161}$ V. Papadimitriou, ${ }^{161}$ K. Pedro, ${ }^{161}$ C. Pena, ${ }^{161, e e e}$ O. Prokofyev, ${ }^{161}$ F. Ravera, ${ }^{161}$ A. Reinsvold Hall, ${ }^{161}$ L. Ristori, ${ }^{161}$ B. Schneider, ${ }^{161}$ E. Sexton-Kennedy, ${ }^{161}$ N. Smith, ${ }^{161}$ A. Soha, ${ }^{161}$ L. Spiegel, ${ }^{161}$ S. Stoynev, ${ }^{161}$ J. Strait, ${ }^{161}$ L. Taylor, ${ }^{161}$ S. Tkaczyk, ${ }^{161}$

N. V. Tran, ${ }^{161}$ L. Uplegger, ${ }^{161}$ E. W. Vaandering, ${ }^{161}$ H. A. Weber, ${ }^{161}$ A. Woodard, ${ }^{161}$ D. Acosta, ${ }^{162}$ P. Avery, ${ }^{162}$ D. Bourilkov, ${ }^{162}$ L. Cadamuro, ${ }^{162}$ V. Cherepanov, ${ }^{162}$ F. Errico, ${ }^{162}$ R. D. Field, ${ }^{162}$ D. Guerrero, ${ }^{162}$ B. M. Joshi, ${ }^{162}$ M. Kim, ${ }^{162}$ J. Konigsberg, ${ }^{162}$ A. Korytov, ${ }^{162}$ K. H. Lo, ${ }^{162}$ K. Matchev, ${ }^{162}$ N. Menendez, ${ }^{162}$ G. Mitselmakher, ${ }^{162}$ D. Rosenzweig, ${ }^{162}$ K. Shi, ${ }^{162}$ J. Sturdy, ${ }^{162}$ J. Wang, ${ }^{162}$ E. Yigitbasi, ${ }^{162}$ X. Zuo, ${ }^{162}$ T. Adams, ${ }^{163}$ A. Askew, ${ }^{163}$ D. Diaz, ${ }^{163}$ R. Habibullah, ${ }^{163}$ S. Hagopian, ${ }^{163}$ V. Hagopian, ${ }^{163}$ K. F. Johnson, ${ }^{163}$ R. Khurana, ${ }^{163}$ T. Kolberg, ${ }^{163}$ G. Martinez, ${ }^{163}$ H. Prosper, ${ }^{163}$ C. Schiber, ${ }^{163}$ R. Yohay, ${ }^{163}$ J. Zhang, ${ }^{163}$ M. M. Baarmand, ${ }^{164}$ S. Butalla, ${ }^{164}$ T. Elkafrawy, ${ }^{164, o}$ M. Hohlmann, ${ }^{164}$ R. Kumar Verma, ${ }^{164}$ D. Noonan, ${ }^{164}$ M. Rahmani, ${ }^{164}$ M. Saunders, ${ }^{164}$ F. Yumiceva, ${ }^{164}$ M. R. Adams, ${ }^{165}$ L. Apanasevich, ${ }^{165}$ H. Becerril Gonzalez, ${ }^{165}$ R. Cavanaugh, ${ }^{165}$ X. Chen, ${ }^{165}$ S. Dittmer, ${ }^{165}$ O. Evdokimov, ${ }^{165}$ C. E. Gerber, ${ }^{165}$ D. A. Hangal, ${ }^{165}$ D. J. Hofman, ${ }^{165}$ C. Mills, ${ }^{165}$ G. Oh, ${ }^{165}$ T. Roy, ${ }^{165}$ M. B. Tonjes, ${ }^{165}$ N. Varelas, ${ }^{165}$ J. Viinikainen, ${ }^{165}$ X. Wang, ${ }^{165}$ Z. Wu, ${ }^{165}$ Z. Ye, ${ }^{165}$ M. Alhusseini, ${ }^{166}$ K. Dilsiz, ${ }^{166, m m m m}$ S. Durgut, ${ }^{166}$ R. P. Gandrajula, ${ }^{166}$ M. Haytmyradov, ${ }^{166}$ V. Khristenko, ${ }^{166}$ O. K. Köseyan, ${ }^{166}$ J.-P. Merlo, ${ }^{166}$ A. Mestvirishvili, ${ }^{166, n n n n}$ A. Moeller, ${ }^{166}$ J. Nachtman, ${ }^{166}$ H. Ogul, ${ }^{166,0000}$ Y. Onel, ${ }^{166}$ F. Ozok, ${ }^{166, \text { pppp }}$ A. Penzo, ${ }^{166}$ C. Snyder, ${ }^{166}$ E. Tiras, ${ }^{166, q q q q}$ J. Wetzel, ${ }^{166}$ O. Amram, ${ }^{167}$ B. Blumenfeld, ${ }^{167}$ L. Corcodilos, ${ }^{167}$

M. Eminizer, ${ }^{167}$ A. V. Gritsan, ${ }^{167}$ S. Kyriacou, ${ }^{167}$ P. Maksimovic, ${ }^{167}$ J. Roskes, ${ }^{167}$ M. Swartz, ${ }^{167}$ T. Á. Vámi, ${ }^{167}$ C. Baldenegro Barrera, ${ }^{168}$ P. Baringer, ${ }^{168}$ A. Bean, ${ }^{168}$ A. Bylinkin, ${ }^{168}$ T. Isidori, ${ }^{168}$ S. Khalil, ${ }^{168}$ J. King, ${ }^{168}$ G. Krintiras, ${ }^{168}$ A. Kropivnitskaya, ${ }^{168}$ C. Lindsey, ${ }^{168}$ N. Minafra, ${ }^{168}$ M. Murray, ${ }^{168}$ C. Rogan, ${ }^{168}$ C. Royon, ${ }^{168}$ S. Sanders, ${ }^{168}$ E. Schmitz, ${ }^{168}$ J. D. Tapia Takaki, ${ }^{168}$ Q. Wang, ${ }^{168}$ J. Williams, ${ }^{168}$ G. Wilson, ${ }^{168}$ S. Duric, ${ }^{169}$ A. Ivanov, ${ }^{169}$ K. Kaadze, ${ }^{169}$ D. Kim, ${ }^{169}$ Y. Maravin, ${ }^{169}$ T. Mitchell, ${ }^{169}$ A. Modak, ${ }^{169}$ K. Nam,${ }^{169}$ F. Rebassoo, ${ }^{170}$ D. Wright, ${ }^{170}$ E. Adams, ${ }^{171}$ A. Baden, ${ }^{171}$ O. Baron, ${ }^{171}$ A. Belloni, ${ }^{171}$ S. C. Eno, ${ }^{171}$ Y. Feng, ${ }^{171}$ N. J. Hadley, ${ }^{171}$ S. Jabeen, ${ }^{171}$ R. G. Kellogg, ${ }^{171}$ T. Koeth, ${ }^{171}$ A. C. Mignerey, ${ }^{171}$ S. Nabili, ${ }^{171}$ M. Seidel, ${ }^{171}$ A. Skuja, ${ }^{171}$ S. C. Tonwar, ${ }^{171}$ L. Wang, ${ }^{171}$ K. Wong, ${ }^{171}$ D. Abercrombie, ${ }^{172}$ G. Andreassi, ${ }^{172}$ R. Bi, ${ }^{172}$ S. Brandt, ${ }^{172}$ W. Busza, ${ }^{172}$ I. A. Cali, ${ }^{172}$ Y. Chen, ${ }^{172}$ M. D' Alfonso, ${ }^{172}$ G. Gomez Ceballos, ${ }^{172}$ M. Goncharov, ${ }^{172}$ P. Harris, ${ }^{172}$ M. Hu, ${ }^{172}$ M. Klute, ${ }^{172}$ D. Kovalskyi, ${ }^{172}$ J. Krupa, ${ }^{172}$ Y.-J. Lee, ${ }^{172}$ B. Maier, ${ }^{172}$ A. C. Marini, ${ }^{172}$ C. Mironov, ${ }^{172}$ C. Paus, ${ }^{172}$ D. Rankin, ${ }^{172}$ C. Roland, ${ }^{172}$ G. Roland, ${ }^{172}$ Z. Shi, ${ }^{172}$ G. S. F. Stephans, ${ }^{172}$ K. Tatar, ${ }^{172}$ J. Wang, ${ }^{172}$ Z. Wang, ${ }^{172}$ B. Wyslouch, ${ }^{172}$ R. M. Chatterjee, ${ }^{173}$ A. Evans, ${ }^{173}$ P. Hansen, ${ }^{173}$ J. Hiltbrand, ${ }^{173}$ Sh. Jain, ${ }^{173}$ M. Krohn, ${ }^{173}$ Y. Kubota, ${ }^{173}$ Z. Lesko, ${ }^{173}$ J. Mans, ${ }^{173}$ M. Revering, ${ }^{173}$ R. Rusack, ${ }^{173}$ R. Saradhy, ${ }^{173}$

N. Schroeder, ${ }^{173}$ N. Strobbe, ${ }^{173}$ M. A. Wadud, ${ }^{173}$ J. G. Acosta, ${ }^{174}$ S. Oliveros, ${ }^{174}$ K. Bloom, ${ }^{175}$ M. Bryson, ${ }^{175}$ S. Chauhan, ${ }^{175}$ D. R. Claes, ${ }^{175}$ C. Fangmeier, ${ }^{175}$ L. Finco, ${ }^{175}$ F. Golf, ${ }^{175}$ J. R. González Fernández, ${ }^{175}$ C. Joo, ${ }^{175}$ I. Kravchenko, ${ }^{175}$ J. E. Siado, ${ }^{175}$ G. R. Snow, ${ }^{175, a}$ W. Tabb, ${ }^{175}$ F. Yan, ${ }^{175}$ G. Agarwal, ${ }^{176}$ H. Bandyopadhyay, ${ }^{176}$ L. Hay, ${ }^{176}$ I. Iashvili, ${ }^{176}$ A. Kharchilava, ${ }^{176}$ C. McLean, ${ }^{176}$ D. Nguyen, ${ }^{176}$ J. Pekkanen, ${ }^{176}$ S. Rappoccio, ${ }^{176}$ A. Williams, ${ }^{176}$ G. Alverson, ${ }^{177}$ E. Barberis, ${ }^{177}$ C. Freer, ${ }^{177}$ Y. Haddad, ${ }^{177}$ A. Hortiangtham, ${ }^{177}$ J. Li, ${ }^{177}$ G. Madigan, ${ }^{177}$ B. Marzocchi, ${ }^{177}$ D. M. Morse, ${ }^{177}$ V. Nguyen, ${ }^{177}$ T. Orimoto, ${ }^{177}$ A. Parker, ${ }^{177}$ L. Skinnari, ${ }^{177}$ A. Tishelman-Charny, ${ }^{177}$ T. Wamorkar, ${ }^{177}$ B. Wang, ${ }^{177}$

A. Wisecarver, ${ }^{177}$ D. Wood, ${ }^{177}$ S. Bhattacharya, ${ }^{178}$ J. Bueghly, ${ }^{178}$ Z. Chen, ${ }^{178}$ A. Gilbert, ${ }^{178}$ T. Gunter, ${ }^{178}$ K. A. Hahn, ${ }^{178}$ N. Odell, ${ }^{178}$ M. H. Schmitt, ${ }^{178}$ K. Sung, ${ }^{178}$ M. Velasco, ${ }^{178}$ R. Band, ${ }^{179}$ R. Bucci, ${ }^{179}$ N. Dev, ${ }^{179}$ R. Goldouzian, ${ }^{179}$ M. Hildreth, ${ }^{179}$ K. Hurtado Anampa, ${ }^{179}$ C. Jessop, ${ }^{179}$ K. Lannon, ${ }^{179}$ N. Loukas, ${ }^{179}$ N. Marinelli, ${ }^{179}$ I. Mcalister, ${ }^{179}$ F. Meng, ${ }^{179}$ K. Mohrman, ${ }^{179}$ Y. Musienko, ${ }^{179, x x}$ R. Ruchti, ${ }^{179}$ P. Siddireddy, ${ }^{179}$ M. Wayne, ${ }^{179}$ A. Wightman, ${ }^{179}$ M. Wolf, ${ }^{179}$ M. Zarucki, ${ }^{179}$ L. Zygala, ${ }^{179}$ B. Bylsma, ${ }^{180}$ B. Cardwell, ${ }^{180}$ L. S. Durkin, ${ }^{180}$ B. Francis, ${ }^{180}$ C. Hill, ${ }^{180}$ A. Lefeld, ${ }^{180}$ B. L. Winer, ${ }^{180}$ B. R. Yates, ${ }^{180}$ F. M. Addesa, ${ }^{181}$ B. Bonham, ${ }^{181}$ P. Das, ${ }^{181}$ G. Dezoort, ${ }^{181}$ P. Elmer, ${ }^{181}$ A. Frankenthal, ${ }^{181}$ B. Greenberg, ${ }^{181}$ N. Haubrich, ${ }^{181}$ S. Higginbotham, ${ }^{181}$ A. Kalogeropoulos, ${ }^{181}$ G. Kopp, ${ }^{181}$ S. Kwan, ${ }^{181}$ D. Lange, ${ }^{181}$ M. T. Lucchini, ${ }^{181}$ D. Marlow, ${ }^{181}$ K. Mei, ${ }^{181}$ I. Ojalvo, ${ }^{181}$ J. Olsen, ${ }^{181}$ C. Palmer, ${ }^{181}$ D. Stickland, ${ }^{181}$ C. Tully, ${ }^{181}$ S. Malik, ${ }^{182}$ S. Norberg, ${ }^{182}$ A. S. Bakshi, ${ }^{183}$ V. E. Barnes, ${ }^{183}$ R. Chawla, ${ }^{183}$ S. Das, ${ }^{183}$ L. Gutay, ${ }^{183}$ M. Jones, ${ }^{183}$ A. W. Jung, ${ }^{183}$ S. Karmarkar, ${ }^{183}$ M. Liu, ${ }^{183}$ G. Negro, ${ }^{183}$ N. Neumeister, ${ }^{183}$ G. Paspalaki, ${ }^{183}$ C. C. Peng, ${ }^{183}$ S. Piperov, ${ }^{183}$ A. Purohit, ${ }^{183}$ J. F. Schulte, ${ }^{183}$ M. Stojanovic, ${ }^{183, \mathrm{r}}$ J. Thieman, ${ }^{183}$ F. Wang, ${ }^{183}$ R. Xiao, ${ }^{183}$ W. Xie, ${ }^{183}$ J. Dolen, ${ }^{184}$ N. Parashar, ${ }^{184}$ A. Baty, ${ }^{185}$ S. Dildick, ${ }^{185}$ K. M. Ecklund, ${ }^{185}$ S. Freed, ${ }^{185}$ F. J. M. Geurts, ${ }^{185}$ A. Kumar, ${ }^{185}$ W. Li, ${ }^{185}$ B. P. Padley, ${ }^{185}$ R. Redjimi, ${ }^{185}$ J. Roberts, ${ }^{185, a}$ W. Shi, ${ }^{185}$ A. G. Stahl Leiton, ${ }^{185}$ A. Bodek, ${ }^{186}$ P. de Barbaro, ${ }^{186}$ R. Demina, ${ }^{186}$ J. L. Dulemba, ${ }^{186}$ C. Fallon, ${ }^{186}$ T. Ferbel, ${ }^{186}$ M. Galanti, ${ }^{186}$ A. Garcia-Bellido, ${ }^{186}$ O. Hindrichs, ${ }^{186}$ A. Khukhunaishvili, ${ }^{186}$ E. Ranken, ${ }^{186}$ R. Taus, ${ }^{186}$ 
B. Chiarito, ${ }^{187}$ J. P. Chou, ${ }^{187}$ A. Gandrakota, ${ }^{187}$ Y. Gershtein, ${ }^{187}$ E. Halkiadakis, ${ }^{187}$ A. Hart, ${ }^{187}$ M. Heindl, ${ }^{187}$ E. Hughes, ${ }^{187}$ S. Kaplan, ${ }^{187}$ O. Karacheban, ${ }^{187, y}$ I. Laflotte, ${ }^{187}$ A. Lath, ${ }^{187}$ R. Montalvo, ${ }^{187}$ K. Nash, ${ }^{187}$ M. Osherson, ${ }^{187}$ S. Salur, ${ }^{187}$ S. Schnetzer, ${ }^{187}$ S. Somalwar, ${ }^{187}$ R. Stone, ${ }^{187}$ S. A. Thayil, ${ }^{187}$ S. Thomas, ${ }^{187}$ H. Wang, ${ }^{187}$ H. Acharya,${ }^{188}$ A. G. Delannoy, ${ }^{188}$ S. Spanier, ${ }^{188}$ O. Bouhali, ${ }^{189, \text { rrrr }}$ M. Dalchenko, ${ }^{189}$ A. Delgado, ${ }^{189}$ R. Eusebi, ${ }^{189}$ J. Gilmore, ${ }^{189}$ T. Huang, ${ }^{189}$ T. Kamon, ${ }^{189, \text { ssss }}$ H. Kim, ${ }^{189}$ S. Luo, ${ }^{189}$ S. Malhotra, ${ }^{189}$ R. Mueller, ${ }^{189}$ D. Overton, ${ }^{189}$ D. Rathjens, ${ }^{189}$ A. Safonov, ${ }^{189}$ N. Akchurin, ${ }^{190}$ J. Damgov, ${ }^{190}$ V. Hegde, ${ }^{190}$ S. Kunori,${ }^{190}$ K. Lamichhane, ${ }^{190}$ S. W. Lee ${ }^{190}$ T. Mengke, ${ }^{190}$ S. Muthumuni,${ }^{190}$ T. Peltola, ${ }^{190}$ S. Undleeb, ${ }^{190}$ I. Volobouev, ${ }^{190}$ Z. Wang, ${ }^{190}$ A. Whitbeck, ${ }^{190}$ E. Appelt, ${ }^{191}$ S. Greene, ${ }^{191}$ A. Gurrola, ${ }^{191}$ W. Johns, ${ }^{191}$ C. Maguire, ${ }^{191}$ A. Melo, ${ }^{191}$ H. Ni, ${ }^{191}$ K. Padeken, ${ }^{191}$ F. Romeo ${ }^{191}$ P. Sheldon, ${ }^{191}$ S. Tuo, ${ }^{191}$ J. Velkovska, ${ }^{191}$ M. W. Arenton, ${ }^{192}$ B. Cox,${ }^{192}$ G. Cummings, ${ }^{192}$ J. Hakala, ${ }^{192}$ R. Hirosky, ${ }^{192}$ M. Joyce, ${ }^{192}$ A. Ledovskoy, ${ }^{192}$ A. Li,${ }^{192}$ C. Neu, ${ }^{192}$ B. Tannenwald, ${ }^{192}$ E. Wolfe, ${ }^{192}$ P. E. Karchin, ${ }^{193}$ N. Poudyal,,${ }^{193}$ P. Thapa,${ }^{193}$ K. Black, ${ }^{194}$ T. Bose,${ }^{194}$ J. Buchanan, ${ }^{194}$ C. Caillol, ${ }^{194}$ S. Dasu, ${ }^{194}$ I. De Bruyn, ${ }^{194}$ P. Everaerts, ${ }^{194}$ F. Fienga, ${ }^{194}$ C. Galloni, ${ }^{194}$ H. He,${ }^{194}$ M. Herndon, ${ }^{194}$ A. Hervé, ${ }^{194}$ U. Hussain, ${ }^{194}$ A. Lanaro, ${ }^{194}$ A. Loeliger, ${ }^{194}$ R. Loveless, ${ }^{194}$ J. Madhusudanan Sreekala, ${ }^{194}$ A. Mallampalli, ${ }^{194}$ A. Mohammadi, ${ }^{194}$ D. Pinna, ${ }^{194}$ A. Savin, ${ }^{194}$ V. Shang, ${ }^{194}$ V. Sharma, ${ }^{194}$ W. H. Smith, ${ }^{194}$ D. Teague, ${ }^{194}$ S. Trembath-Reichert, ${ }^{194}$ and W. Vetens ${ }^{194}$

\title{
(CMS Collaboration)
}

\author{
${ }^{1}$ Yerevan Physics Institute, Yerevan, Armenia \\ ${ }^{2}$ Institut für Hochenergiephysik, Wien, Austria \\ ${ }^{3}$ Institute for Nuclear Problems, Minsk, Belarus \\ ${ }^{4}$ Universiteit Antwerpen, Antwerpen, Belgium \\ ${ }^{5}$ Vrije Universiteit Brussel, Brussel, Belgium \\ ${ }^{6}$ Université Libre de Bruxelles, Bruxelles, Belgium \\ ${ }^{7}$ Ghent University, Ghent, Belgium \\ ${ }^{8}$ Université Catholique de Louvain, Louvain-la-Neuve, Belgium \\ ${ }^{9}$ Centro Brasileiro de Pesquisas Fisicas, Rio de Janeiro, Brazil \\ ${ }^{10}$ Universidade do Estado do Rio de Janeiro, Rio de Janeiro, Brazil \\ ${ }^{11 a}$ Universidade Estadual Paulista, São Paulo, Brazil \\ ${ }^{11 \mathrm{~b}}$ Universidade Federal do ABC, São Paulo, Brazil \\ ${ }^{12}$ Institute for Nuclear Research and Nuclear Energy, Bulgarian Academy of Sciences, Sofia, Bulgaria \\ ${ }^{13}$ University of Sofia, Sofia, Bulgaria \\ ${ }^{14}$ Beihang University, Beijing, China \\ ${ }^{15}$ Department of Physics, Tsinghua University, Beijing, China \\ ${ }^{16}$ Institute of High Energy Physics, Beijing, China \\ ${ }^{17}$ State Key Laboratory of Nuclear Physics and Technology, Peking University, Beijing, China \\ ${ }^{18}$ Sun Yat-Sen University, Guangzhou, China \\ ${ }^{19}$ Institute of Modern Physics and Key Laboratory of Nuclear Physics and Ion-beam Application (MOE)- \\ Fudan University, Shanghai, China \\ ${ }^{20}$ Zhejiang University, Hangzhou, China \\ ${ }^{21}$ Universidad de Los Andes, Bogota, Colombia \\ ${ }^{22}$ Universidad de Antioquia, Medellin, Colombia \\ ${ }^{23}$ University of Split, Faculty of Electrical Engineering, Mechanical Engineering and Naval Architecture, \\ Split, Croatia \\ ${ }^{24}$ University of Split, Faculty of Science, Split, Croatia \\ ${ }^{25}$ Institute Rudjer Boskovic, Zagreb, Croatia \\ ${ }^{26}$ University of Cyprus, Nicosia, Cyprus \\ ${ }^{27}$ Charles University, Prague, Czech Republic \\ ${ }^{28}$ Escuela Politecnica Nacional, Quito, Ecuador \\ ${ }^{29}$ Universidad San Francisco de Quito, Quito, Ecuador \\ ${ }^{30}$ Academy of Scientific Research and Technology of the Arab Republic of Egypt, \\ Egyptian Network of High Energy Physics, Cairo, Egypt \\ ${ }^{31}$ Center for High Energy Physics (CHEP-FU), Fayoum University, El-Fayoum, Egypt \\ ${ }^{32}$ National Institute of Chemical Physics and Biophysics, Tallinn, Estonia \\ ${ }^{33}$ Department of Physics, University of Helsinki, Helsinki, Finland \\ ${ }^{34}$ Helsinki Institute of Physics, Helsinki, Finland \\ ${ }^{35}$ Lappeenranta University of Technology, Lappeenranta, Finland
}


${ }^{36}$ IRFU, CEA, Université Paris-Saclay, Gif-sur-Yvette, France

${ }^{37}$ Laboratoire Leprince-Ringuet, CNRS/IN2P3, Ecole Polytechnique, Institut Polytechnique de Paris, Palaiseau, France

${ }^{38}$ Université de Strasbourg, CNRS, IPHC UMR 7178, Strasbourg, France

${ }^{39}$ Institut de Physique des 2 Infinis de Lyon (IP2I), Villeurbanne, France

${ }^{40}$ Georgian Technical University, Tbilisi, Georgia

${ }^{41}$ RWTH Aachen University, I. Physikalisches Institut, Aachen, Germany

${ }^{42}$ RWTH Aachen University, III. Physikalisches Institut A, Aachen, Germany

${ }^{43}$ RWTH Aachen University, III. Physikalisches Institut B, Aachen, Germany

${ }^{44}$ Deutsches Elektronen-Synchrotron, Hamburg, Germany

${ }^{45}$ University of Hamburg, Hamburg, Germany

${ }^{46}$ Karlsruher Institut fuer Technologie, Karlsruhe, Germany

${ }^{47}$ Institute of Nuclear and Particle Physics (INPP), NCSR Demokritos, Aghia Paraskevi, Greece

${ }^{48}$ National and Kapodistrian University of Athens, Athens, Greece

${ }^{49}$ National Technical University of Athens, Athens, Greece

${ }^{50}$ University of Ioánnina, Ioánnina, Greece

${ }^{51}$ MTA-ELTE Lendület CMS Particle and Nuclear Physics Group, Eötvös Loránd University, Budapest, Hungary

${ }^{52}$ Wigner Research Centre for Physics, Budapest, Hungary

${ }^{53}$ Institute of Nuclear Research ATOMKI, Debrecen, Hungary

${ }^{54}$ Institute of Physics, University of Debrecen, Debrecen, Hungary

${ }^{55}$ Eszterhazy Karoly University, Karoly Robert Campus, Gyongyos, Hungary

${ }^{56}$ Indian Institute of Science (IISc), Bangalore, India

${ }^{57}$ National Institute of Science Education and Research, HBNI, Bhubaneswar, India

${ }^{58}$ Panjab University, Chandigarh, India

${ }^{59}$ University of Delhi, Delhi, India

${ }^{60}$ Saha Institute of Nuclear Physics, HBNI, Kolkata, India

${ }^{61}$ Indian Institute of Technology Madras, Madras, India

${ }^{62}$ Bhabha Atomic Research Centre, Mumbai, India

${ }^{63}$ Tata Institute of Fundamental Research-A, Mumbai, India

${ }^{64}$ Tata Institute of Fundamental Research-B, Mumbai, India

${ }^{65}$ Indian Institute of Science Education and Research (IISER), Pune, India

${ }^{66}$ Department of Physics, Isfahan University of Technology, Isfahan, Iran

${ }^{67}$ Institute for Research in Fundamental Sciences (IPM), Tehran, Iran

${ }^{68}$ University College Dublin, Dublin, Ireland

${ }^{69 a}$ INFN Sezione di Bari, Bari, Italy

${ }^{69 \mathrm{~b}}$ Università di Bari, Bari, Italy

${ }^{69 \mathrm{c}}$ Politecnico di Bari, Bari, Italy

${ }^{70 a}$ INFN Sezione di Bologna, Bologna, Italy

${ }^{70 \mathrm{~b}}$ Università di Bologna, Bologna, Italy

${ }^{71 a}$ INFN Sezione di Catania

${ }^{71 b}$ Università di Catania

${ }^{72 \mathrm{a}}$ INFN Sezione di Firenze, Firenze, Italy

${ }^{72 \mathrm{~b}}$ Università di Firenze, Firenze, Italy

${ }^{73}$ INFN Laboratori Nazionali di Frascati, Frascati, Italy

${ }^{74 a}$ INFN Sezione di Genova

${ }^{74 \mathrm{~b}}$ Università di Genova

${ }^{75 a}$ INFN Sezione di Milano-Bicocca, Milano, Italy

${ }^{75 b}$ Università di Milano-Bicocca, Milano, Italy

${ }^{76 a}$ INFN Sezione di Napoli, Napoli, Italy

${ }^{76 \mathrm{~b}}$ Università di Napoli 'Federico II', Napoli, Italy

${ }^{76 c}$ Università della Basilicata, Napoli, Italy

${ }^{76 \mathrm{~d}}$ Università G. Marconi

${ }^{77 a}$ INFN Sezione di Padova

${ }^{77 \mathrm{~b}}$ Università di Padova

${ }^{77 \mathrm{c}}$ Università di Trento

${ }^{78 a}$ INFN Sezione di Pavia, Pavia, Italy

${ }^{78 b}$ Università di Pavia, Pavia, Italy

${ }^{79 a}$ INFN Sezione di Perugia, Perugia, Italy

${ }^{79 b}$ Università di Perugia, Perugia, Italy 


\author{
${ }^{80 a}$ INFN Sezione di Pisa, Pisa, Italy \\ ${ }^{80 \mathrm{~b}}$ Università di Pisa, Pisa, Italy \\ ${ }^{80 c}$ Scuola Normale Superiore di Pisa, Pisa, Italy \\ ${ }^{80 \mathrm{~d}}$ Università di Siena, Siena, Italy \\ ${ }^{81 a}$ INFN Sezione di Roma, Roma, Italy \\ ${ }^{81 \mathrm{~b}}$ Sapienza Università di Roma, Roma, Italy \\ ${ }^{82 \mathrm{a} I N F N}$ Sezione di Torino, Torino, Italy \\ ${ }^{82 \mathrm{~b}}$ Università di Torino, Torino, Italy \\ ${ }^{82 \mathrm{c}}$ Università del Piemonte Orientale, Novara, Italy \\ ${ }^{83 a}$ INFN Sezione di Trieste, Trieste, Italy \\ ${ }^{83 \mathrm{~b}}$ Università di Trieste, Trieste, Italy \\ ${ }^{84}$ Kyungpook National University, Daegu, Korea \\ ${ }^{85}$ Chonnam National University, Institute for Universe and Elementary Particles, Kwangju, Korea \\ ${ }^{86}$ Hanyang University, Seoul, Korea \\ ${ }^{87}$ Korea University, Seoul, Korea \\ ${ }^{88}$ Kyung Hee University, Department of Physics, Seoul, Republic of Korea \\ ${ }^{89}$ Sejong University, Seoul, Korea \\ ${ }^{90}$ Seoul National University, Seoul, Korea \\ ${ }^{91}$ University of Seoul, Seoul, Korea \\ ${ }^{92}$ Yonsei University, Department of Physics, Seoul, Korea \\ ${ }^{93}$ Sungkyunkwan University, Suwon, Korea \\ ${ }^{94}$ College of Engineering and Technology, American University of the Middle East (AUM), Egaila, Kuwait \\ ${ }^{95}$ Riga Technical University, Riga, Latvia \\ ${ }^{96}$ Vilnius University, Vilnius, Lithuania \\ ${ }^{97}$ National Centre for Particle Physics, Universiti Malaya, Kuala Lumpur, Malaysia \\ ${ }^{98}$ Universidad de Sonora (UNISON), Hermosillo, Mexico \\ ${ }^{99}$ Centro de Investigacion y de Estudios Avanzados del IPN, Mexico City, Mexico \\ ${ }^{100}$ Universidad Iberoamericana, Mexico City, Mexico \\ ${ }^{101}$ Benemerita Universidad Autonoma de Puebla, Puebla, Mexico \\ ${ }^{102}$ University of Montenegro, Podgorica, Montenegro \\ ${ }^{103}$ University of Auckland, Auckland, New Zealand \\ ${ }^{104}$ University of Canterbury, Christchurch, New Zealand \\ ${ }^{105}$ National Centre for Physics, Quaid-I-Azam University, Islamabad, Pakistan \\ ${ }^{106}$ AGH University of Science and Technology Faculty of Computer Science, \\ Electronics and Telecommunications, Krakow, Poland \\ ${ }^{107}$ National Centre for Nuclear Research, Swierk, Poland \\ ${ }^{108}$ Institute of Experimental Physics, Faculty of Physics, University of Warsaw, Warsaw, Poland \\ ${ }^{109}$ Laboratório de Instrumentação e Física Experimental de Partículas, Lisboa, Portugal \\ ${ }^{110}$ Joint Institute for Nuclear Research, Dubna, Russia \\ ${ }^{111}$ Petersburg Nuclear Physics Institute, Gatchina (St. Petersburg), Russia \\ ${ }^{112}$ Institute for Nuclear Research, Moscow, Russia \\ ${ }^{113}$ Institute for Theoretical and Experimental Physics \\ named by A.I. Alikhanov of NRC 'Kurchatov Institute', Moscow, Russia \\ ${ }^{114}$ Moscow Institute of Physics and Technology, Moscow, Russia \\ ${ }^{115}$ National Research Nuclear University 'Moscow Engineering Physics Institute' (MEPhI), \\ Moscow, Russia \\ ${ }^{116}$ P.N. Lebedev Physical Institute, Moscow, Russia \\ ${ }^{117}$ Skobeltsyn Institute of Nuclear Physics, Lomonosov Moscow State University, Moscow, Russia \\ ${ }^{118}$ Novosibirsk State University (NSU), Novosibirsk, Russia \\ ${ }^{119}$ Institute for High Energy Physics of National Research Centre 'Kurchatov Institute', Protvino, Russia \\ ${ }^{120}$ National Research Tomsk Polytechnic University, Tomsk, Russia \\ ${ }^{121}$ Tomsk State University, Tomsk, Russia \\ ${ }^{122}$ University of Belgrade: Faculty of Physics and VINCA Institute of Nuclear Sciences, Belgrade, Serbia \\ ${ }^{123}$ Centro de Investigaciones Energéticas Medioambientales y Tecnológicas (CIEMAT), Madrid, Spain \\ ${ }^{124}$ Universidad Autónoma de Madrid, Madrid, Spain \\ ${ }^{125}$ Universidad de Oviedo, Instituto Universitario de Ciencias y Tecnologías \\ Espaciales de Asturias (ICTEA), Oviedo, Spain \\ ${ }^{126}$ Instituto de Física de Cantabria (IFCA), CSIC-Universidad de Cantabria, Santander, Spain \\ ${ }^{127}$ University of Colombo, Colombo, Sri Lanka \\ ${ }^{128}$ University of Ruhuna, Department of Physics, Matara, Sri Lanka
}




\author{
${ }^{129}$ CERN, European Organization for Nuclear Research, Geneva, Switzerland \\ ${ }^{130}$ Paul Scherrer Institut, Villigen, Switzerland \\ ${ }^{131}$ ETH Zurich-Institute for Particle Physics and Astrophysics (IPA), Zurich, Switzerland \\ ${ }^{132}$ Universität Zürich, Zurich, Switzerland \\ ${ }^{133}$ National Central University, Chung-Li, Taiwan \\ ${ }^{134}$ National Taiwan University (NTU), Taipei, Taiwan \\ ${ }^{135}$ Chulalongkorn University, Faculty of Science, Department of Physics, Bangkok, Thailand \\ ${ }^{136}$ Cukurova University, Physics Department, Science and Art Faculty, Adana, Turkey \\ ${ }^{137}$ Middle East Technical University, Physics Department, Ankara, Turkey \\ ${ }^{138}$ Bogazici University, Istanbul, Turkey \\ ${ }^{139}$ Istanbul Technical University, Istanbul, Turkey \\ ${ }^{140}$ Istanbul University, Istanbul, Turkey \\ ${ }^{141}$ Institute for Scintillation Materials of National Academy of Science of Ukraine, Kharkov, Ukraine \\ ${ }^{142}$ National Scientific Center, Kharkov Institute of Physics and Technology, Kharkov, Ukraine \\ ${ }^{143}$ University of Bristol, Bristol, United Kingdom \\ ${ }^{144}$ Rutherford Appleton Laboratory, Didcot, United Kingdom \\ ${ }^{145}$ Imperial College, London, United Kingdom \\ ${ }^{146}$ Brunel University, Uxbridge, United Kingdom \\ ${ }^{147}$ Baylor University, Waco, Texas, USA \\ ${ }^{148}$ Catholic University of America, Washington, D.C., USA \\ ${ }^{149}$ The University of Alabama, Tuscaloosa, Alabama, USA \\ ${ }^{150}$ Boston University, Boston, Massachusetts, USA \\ ${ }^{151}$ Brown University, Providence, Rhode Island, USA \\ ${ }^{152}$ University of California, Davis, Davis, California, USA \\ ${ }^{153}$ University of California, Los Angeles, California, USA \\ ${ }^{154}$ University of California, Riverside, Riverside, California, USA \\ ${ }^{155}$ University of California, San Diego, La Jolla, California, USA \\ ${ }^{156}$ University of California, Santa Barbara-Department of Physics, Santa Barbara, California, USA \\ ${ }^{157}$ California Institute of Technology, Pasadena, California, USA \\ ${ }^{158}$ Carnegie Mellon University, Pittsburgh, Pennsylvania, USA \\ ${ }^{159}$ University of Colorado Boulder, Boulder, Colorado, USA \\ ${ }^{160}$ Cornell University, Ithaca, New York, USA \\ ${ }^{161}$ Fermi National Accelerator Laboratory, Batavia, Illinois, USA \\ ${ }^{162}$ University of Florida, Gainesville, Florida, USA \\ ${ }^{163}$ Florida State University, Tallahassee, Florida, USA \\ ${ }^{164}$ Florida Institute of Technology, Melbourne, Florida, USA \\ ${ }^{165}$ University of Illinois at Chicago (UIC), Chicago, Illinois, USA \\ ${ }^{166}$ The University of Iowa, Iowa City, Iowa, USA \\ ${ }^{167}$ Johns Hopkins University, Baltimore, Maryland, USA \\ ${ }^{168}$ The University of Kansas, Lawrence, Kansas, USA \\ ${ }^{169}$ Kansas State University, Manhattan, Kansas, USA \\ ${ }^{170}$ Lawrence Livermore National Laboratory, Livermore, California, USA \\ ${ }^{171}$ University of Maryland, College Park, Maryland, USA \\ ${ }^{172}$ Massachusetts Institute of Technology, Cambridge, Massachusetts, USA \\ ${ }^{173}$ University of Minnesota, Minneapolis, Minnesota, USA \\ ${ }^{174}$ University of Mississippi, Oxford, Mississippi, USA \\ ${ }^{175}$ University of Nebraska-Lincoln, Lincoln, Nebraska, USA \\ ${ }^{176}$ State University of New York at Buffalo, Buffalo, New York, USA \\ ${ }^{177}$ Northeastern University, Boston, Massachusetts, USA \\ ${ }^{178}$ Northwestern University, Evanston, Illinois, USA \\ ${ }^{179}$ University of Notre Dame, Notre Dame, Indiana, USA \\ ${ }^{180}$ The Ohio State University, Columbus, Ohio, USA \\ ${ }^{181}$ Princeton University, Princeton, New Jersey, USA \\ ${ }^{182}$ University of Puerto Rico, Mayaguez, Puerto Rico, USA \\ ${ }^{183}$ Purdue University, West Lafayette, Indiana, USA \\ ${ }^{184}$ Purdue University Northwest, Hammond, Indiana, USA \\ ${ }^{185}$ Rice University, Houston, Texas, USA \\ ${ }^{186}$ University of Rochester, Rochester, New York, USA \\ ${ }^{187}$ Rutgers, The State University of New Jersey, Piscataway, New Jersey, USA \\ ${ }^{188}$ University of Tennessee, Knoxville, Tennessee, USA
}




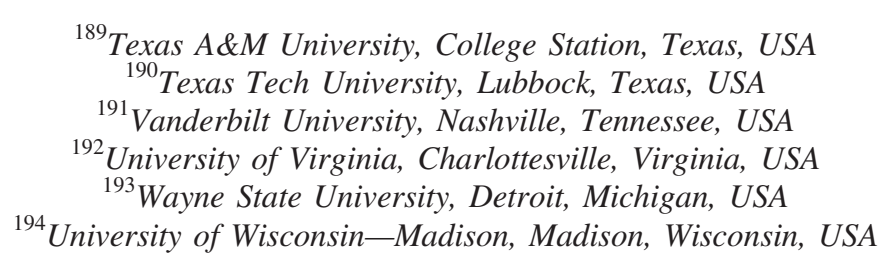

${ }^{\mathrm{a}}$ Deceased.

${ }^{\mathrm{b}}$ Also at Vienna University of Technology, Vienna, Austria.

${ }^{c}$ Also at Institute of Basic and Applied Sciences, Faculty of Engineering, Arab Academy for Science, Technology and Maritime Transport, Alexandria, Egypt.

${ }^{\mathrm{d}}$ Also at Université Libre de Bruxelles, Bruxelles, Belgium.

${ }^{\mathrm{e}}$ Also at Universidade Estadual de Campinas, Campinas, Brazil.

${ }^{\mathrm{f}}$ Also at Federal University of Rio Grande do Sul, Porto Alegre, Brazil.

${ }^{\mathrm{g}}$ Also at University of Chinese Academy of Sciences.

${ }^{\mathrm{h}}$ Also at Department of Physics, Tsinghua University, Beijing, China.

${ }^{\mathrm{i}}$ Universidade Federal do Mato Grosso do Sul, Nova Andradina, Mato Grosso do Sul, Brazil [Universidade Federal do Mato Grosso do Sul = UFMS].

${ }^{\mathrm{j}}$ Also at The University of Iowa, Iowa City, Iowa, USA.

${ }^{\mathrm{k}}$ Also at Nanjing Normal University Department of Physics, Nanjing, China.

${ }^{1}$ Also at University of Chinese Academy of Sciences, Beijing, China.

${ }^{\mathrm{m}}$ Also at Institute for Theoretical and Experimental Physics named by A.I. Alikhanov of NRC 'Kurchatov Institute', Moscow, Russia.

${ }^{\mathrm{n}}$ Also at Joint Institute for Nuclear Research, Dubna, Russia.

${ }^{\circ}$ Also at Ain Shams University, Cairo, Egypt.

${ }^{\mathrm{p}}$ Also at Zewail City of Science and Technology, Zewail, Egypt.

${ }^{\mathrm{q}}$ Also at British University in Egypt, Cairo, Egypt.

${ }^{\mathrm{r}}$ Also at Purdue University, West Lafayette, Indiana, USA.

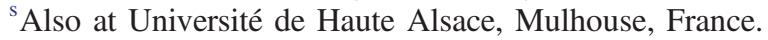

${ }^{\mathrm{t}}$ Also at Erzincan Binali Yildirim University, Erzincan, Turkey.

"Also at CERN, European Organization for Nuclear Research, Geneva, Switzerland.

${ }^{v}$ Also at RWTH Aachen University, III. Physikalisches Institut A, Aachen, Germany.

${ }^{\mathrm{w}}$ Also at University of Hamburg, Hamburg, Germany.

${ }^{\mathrm{x}}$ Also at Department of Physics, Isfahan University of Technology, Isfahan, Iran.

${ }^{y}$ Also at Brandenburg University of Technology, Cottbus, Germany.

${ }^{z}$ Also at Skobeltsyn Institute of Nuclear Physics, Lomonosov Moscow State University, Moscow, Russia.

${ }^{\text {aa } A l s o ~ a t ~ P h y s i c s ~ D e p a r t m e n t, ~ F a c u l t y ~ o f ~ S c i e n c e, ~ A s s i u t ~ U n i v e r s i t y . ~}$

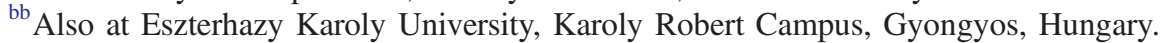

${ }^{c c}$ Also at Institute of Physics, University of Debrecen, Debrecen, Hungary.

${ }^{\mathrm{dd}}$ Also at Institute of Nuclear Research ATOMKI, Debrecen, Hungary.

${ }^{e e}$ Also at MTA-ELTE Lendület CMS Particle and Nuclear Physics Group, Eötvös Loránd University, Budapest, Hungary.

${ }^{\mathrm{ff}}$ Also at Wigner Research Centre for Physics, Budapest, Hungary.

${ }^{\text {gg }}$ Also at IIT Bhubaneswar, Bhubaneswar, India.

${ }^{\text {hh }}$ Also at Institute of Physics, Bhubaneswar, India.

${ }^{\text {ii }}$ Also at G.H.G. Khalsa College, Punjab, India.

${ }^{\mathrm{jj}}$ Also at Shoolini University, Solan, India.

${ }^{\mathrm{kk}}$ Also at University of Hyderabad, Hyderabad, India.

${ }^{11}$ Also at University of Visva-Bharati, Santiniketan, India.

${ }^{\mathrm{mm}}$ Also at Indian Institute of Technology (IIT), Mumbai, India.

${ }^{\mathrm{nn}}$ Also at Deutsches Elektronen-Synchrotron, Hamburg, Germany.

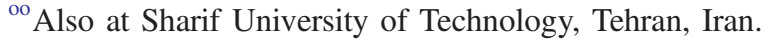

${ }^{\mathrm{pp}}$ Also at Department of Physics, University of Science and Technology of Mazandaran, Behshahr, Iran.

${ }^{\mathrm{qq}}$ Also at INFN Sezione di Bari, Università di Bari, Politecnico di Bari, Bari, Italy.

${ }^{\mathrm{rr}}$ Also at Italian National Agency for New Technologies, Energy and Sustainable Economic Development.

${ }^{\text {ss }}$ Also at Centro Siciliano di Fisica Nucleare e di Struttura Della Materia.

"Also at Università di Napoli 'Federico II', Napoli, Italy.

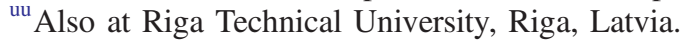

${ }^{\mathrm{vv}}$ Also at Consejo Nacional de Ciencia y Tecnología, Mexico City, Mexico.

${ }^{\text {ww }}$ Also at IRFU, CEA, Université Paris-Saclay, Gif-sur-Yvette, France.

${ }^{\mathrm{xx}}$ Also at Institute for Nuclear Research, Moscow, Russia. 


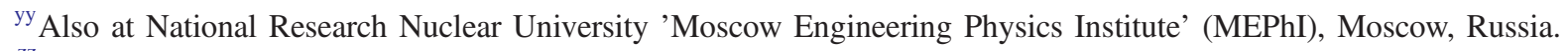

${ }^{\mathrm{zz}}$ Also at St. Petersburg State Polytechnical University, St. Petersburg, Russia.

${ }^{\text {aaa }}$ Also at University of Florida, Gainesville, Florida, USA.

${ }^{b b b}$ Also at Imperial College, London, United Kingdom.

${ }^{c c c}$ Also at P.N. Lebedev Physical Institute, Moscow, Russia.

${ }^{\text {ddd }}$ Also at Moscow Institute of Physics and Technology, Moscow, Russia.

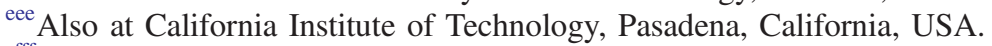

${ }^{\mathrm{fff}}$ Also at Budker Institute of Nuclear Physics, Novosibirsk, Russia.

${ }^{\text {ggg }}$ Also at Faculty of Physics, University of Belgrade, Belgrade, Serbia.

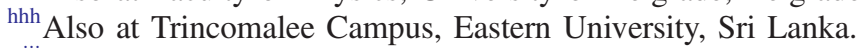

iii Also at INFN Sezione di Pavia, Università di Pavia, Pavia, Italy.

${ }^{\mathrm{jjj}}$ Also at National and Kapodistrian University of Athens, Athens, Greece.

${ }^{k k k}$ Also at Universität Zürich, Zurich, Switzerland.

${ }^{111}$ Also at Ecole Polytechnique Fédérale Lausanne, Lausanne, Switzerland.

${ }^{\mathrm{mmm}}$ Also at Stefan Meyer Institute for Subatomic Physics, Vienna, Austria.

${ }^{n n n}$ Also at Laboratoire d'Annecy-le-Vieux de Physique des Particules, IN2P3-CNRS, Annecy-le-Vieux, France.

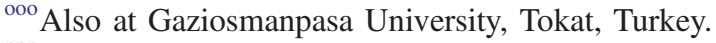

${ }^{\mathrm{ppp}}$ Also at Şırnak University, Şırnak, Turkey.

${ }^{\mathrm{qqq}}$ Also at Near East University, Research Center of Experimental Health Science, Nicosia, Turkey.

${ }^{\mathrm{rrr}}$ Also at Konya Technical University.

${ }^{\text {sss }}$ Also at Istanbul University-Cerraphasa, Faculty of Engineering.

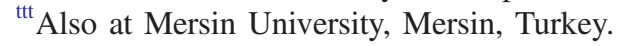

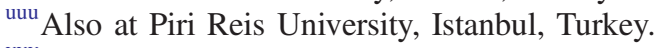

${ }^{\mathrm{vvv}}$ Also at Adiyaman University, Adiyaman, Turkey.

${ }^{\text {www }}$ Also at Tarsus University, Tarsus, Turkey.

${ }^{\mathrm{xxx}}$ Also at Ozyegin University, Istanbul, Turkey.

${ }^{\text {yyy }}$ Also at Izmir Institute of Technology, Izmir, Turkey.

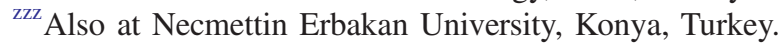

aaaa Also at Bozok Universitetesi Rektörlügü, Yozgat, Turkey.

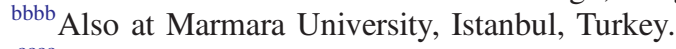

${ }^{\mathrm{cccc}}$ Also at Milli Savunma University, Istanbul, Turkey.

${ }^{\text {dddd }}$ Also at Kafkas University, Kars, Turkey.

${ }^{\text {eee }}$ Also at Istanbul Bilgi University, Istanbul, Turkey.

${ }^{\text {ffff }}$ Also at Hacettepe University, Ankara, Turkey.

${ }^{\text {gggg }}$ Also at Vrije Universiteit Brussel, Brussel, Belgium.

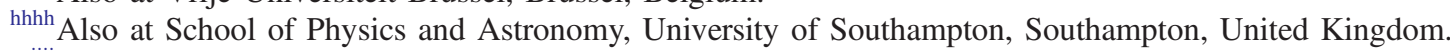

iiii Also at IPPP Durham University, Durham, United Kingdom.

${ }^{j j \mathrm{jj}}$ Also at Monash University, Faculty of Science, Clayton, Australia.

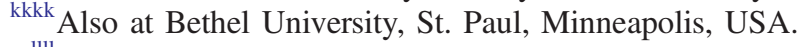

${ }^{1111}$ Also at Karamanoğlu Mehmetbey University, Karaman, Turkey.

${ }^{\mathrm{mmmm}}$ Also at Bingol University, Bingol, Turkey.

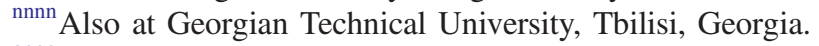

${ }^{\mathrm{oooo}}$ Also at Sinop University, Sinop, Turkey.

${ }^{\text {pppp }}$ Also at Mimar Sinan University, Istanbul, Istanbul, Turkey.

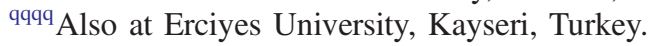

${ }^{\text {rrrr }}$ Also at Texas A\&M University at Qatar, Doha, Qatar.

${ }^{\text {ssss }}$ Also at Kyungpook National University, Daegu, Korea. 